\title{
Application of redox biosensor mouse models to study redox processes in cardiomyocytes
}

\author{
Doctoral thesis \\ for the award of the degree \\ "Doctor of Philosophy" \\ Division of Mathematics and Natural Sciences \\ of the Georg-August-Universität Göttingen \\ in the Molecular medicine doctoral study program \\ of the Georg-August University School of Science (GAUSS)
}

Submitted by

Maithily Nanadikar

Born in

Bhuj, India

Göttingen, 2019 
Thesis Committee

Prof. Dr. Dörthe M. Katschinski (Supervisor)

Institute of Cardiovascular Physiology

University Medical Center, Göttingen

Prof. Dr. Blanche Schwappach-Pignataro

Institute of Molecular Biology

University Medical Center, Göttingen

Prof. Dr. Stephan E. Lehnart

Clinic for Cardiology and Pneumology

University Medical Center, Göttingen

Members of the Examination Board

Dr. Antje Ebert

Clinic for Cardiology and Pneumology

University Medical Center, Göttingen

Prof. Dr. Heidi Hahn

Institute for Human Genetics

University Medical Center, Göttingen

\section{Prof. Hubertus Jarry}

Animal protection commissioner

University Medical Center, Göttingen

Date of oral examination: $11^{\text {th }}$ June 2019 


\section{Affidavit}

I hereby declare that my doctoral thesis entitled "Application of redox biosensor mouse models to study redox processes in cardiomyocytes" has been written independently with no other sources and aids than quoted.

Maithily Nanadikar

Göttingen, April 2019 


\section{List of Publications}

\section{$\mathrm{O}_{2}$ Affects Mitochondrial Functionality ex vivo.}

Nanadikar MS, Vergel Leon AM, Borowik S, Hillemann A, Zieseniss A, Belousov VV, Bogeski I, Rehling P, Dudek J, Katschinski DM. Redox Biol. 2019 Feb 23;22:101152.

Transgenic Organisms Meet Redox Bioimaging: One Step Closer to Physiology.

Swain L, Nanadikar MS, Borowik S, Zieseniss A, Katschinski DM. Antioxid Redox Signal. 2018 Aug 20; 29(6):603-612.

\section{Redox Imaging Using Cardiac Myocyte-Specific Transgenic Biosensor Mice.}

Swain L, Kesemeyer A, Meyer-Roxlau S, Vettel C, Zieseniss A, Güntsch A, Jatho A, Becker A, Nanadikar MS, Morgan B, Dennerlein S, Shah AM, El-Armouche A, Nikolaev VO, Katschinski DM. Circ Res. 2016 Oct 14;119(9):1004-1016. 


\section{Table of contents}

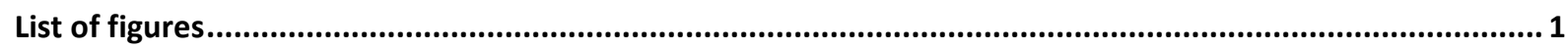

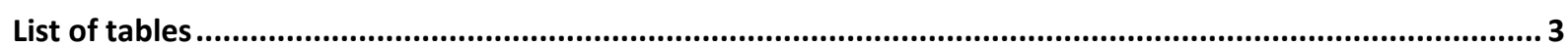

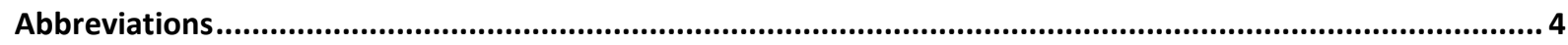

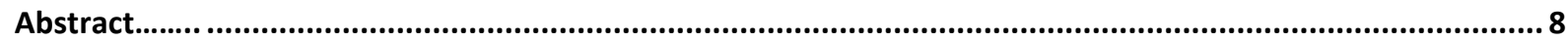

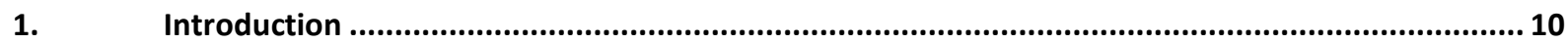

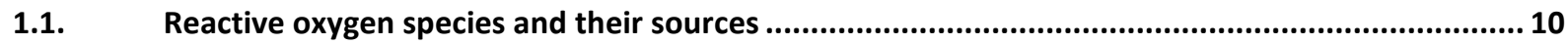

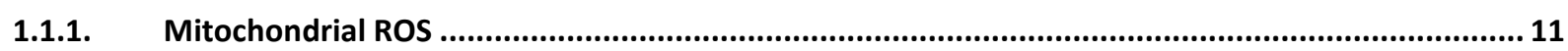

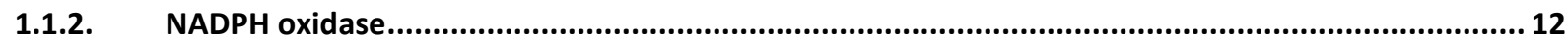

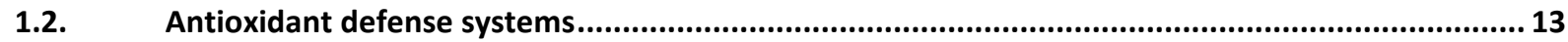

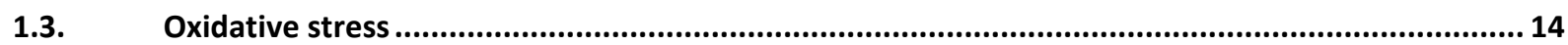

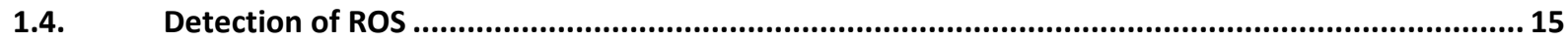

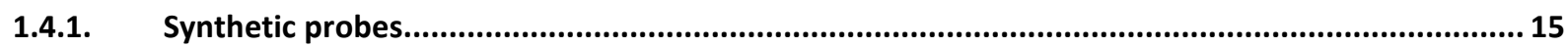

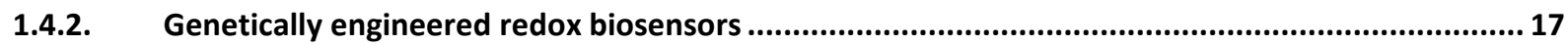

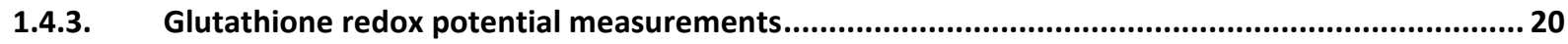

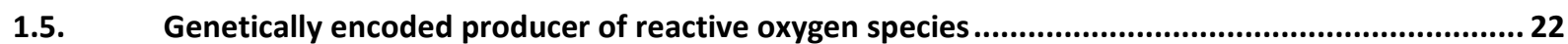

1.6. In vivo models for Grx1-roGFP2 and HyPer based redox biosensors ...................................... 23

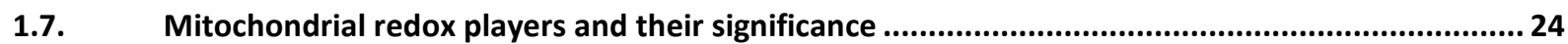

1.8. Molecular oxygen and mitochondria: an ancient relation ................................................26

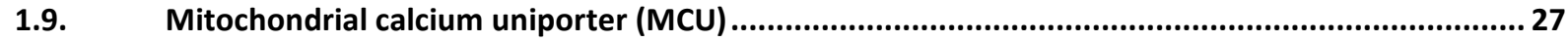

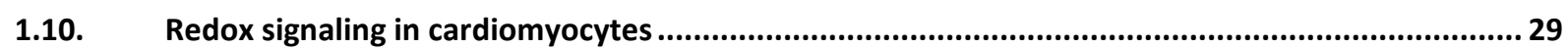

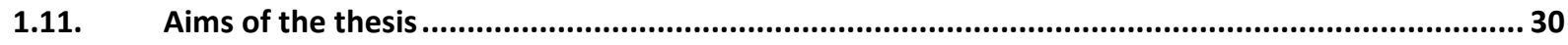

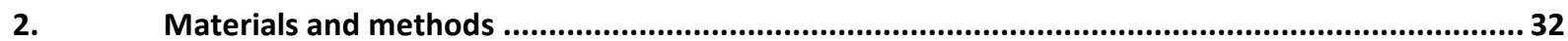




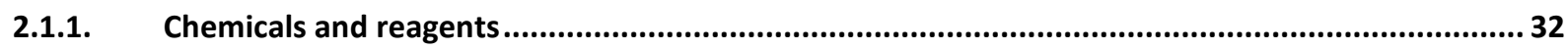

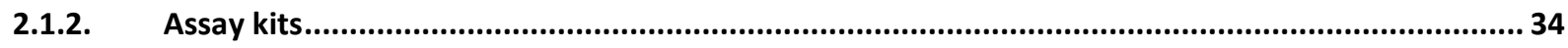

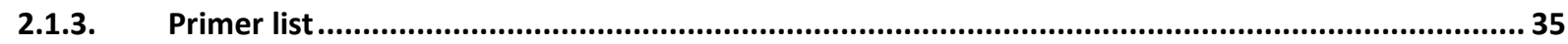

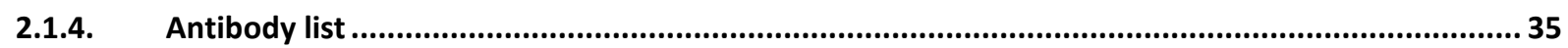

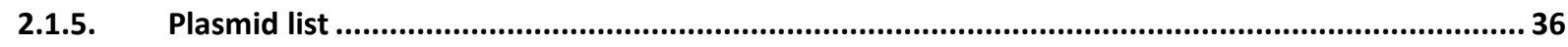

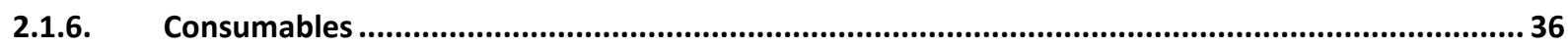

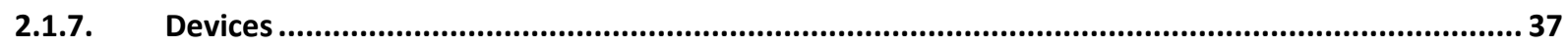

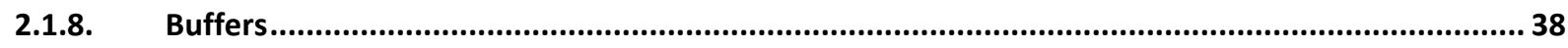

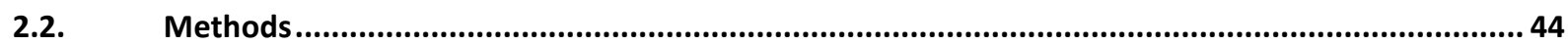

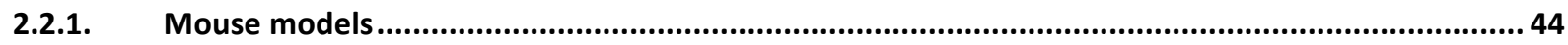

2.2.2. Isolated cardiomyocytes and associated redox analysis ..................................................47

2.2.3. Preparation of cardiac tissue sections and associated analysis ..............................................50

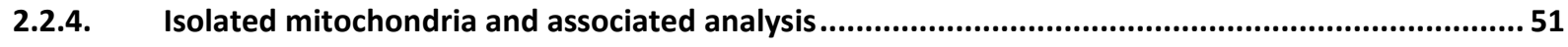

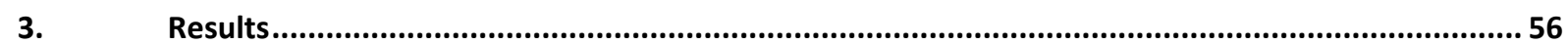

3.1. Ageing affects the compartmentalization of $E_{\mathrm{GSH}}$ in cardiomyocytes ........................................56

3.1.1. Generation of Grx1-roGFP2 biosensor mouse models ................................................... 56

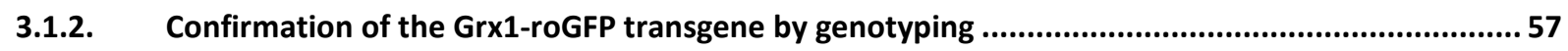

3.1.3. Typical response of isolated Grx1-roGFP2 cardiomyocytes to $\mathrm{H}_{2} \mathrm{O}_{2}$ and DTT .........................58

3.1.4. EGSH of isolated cardiomyocytes from Grx1-roGFP2 mice ................................................59

3.1.5. Echocardiography of ageing mito Grx1-roGFP2 and cyto Grx1-roGFP2 mice as compared to young mito Grx1-roGFP2 and cyto Grx1-roGFP2 mice ...............................................................6 60

3.1.6. $E_{G S H}$ in isolated cardiomyocytes from mito Grx1-roGFP2 and cyto Grx1-roGFP2 aged mice............61

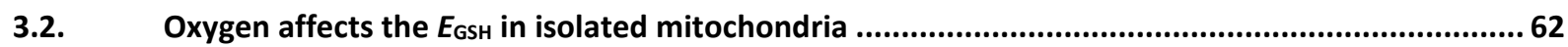


3.2.1. Redox histology performed with heart sections from mito Grx1roGFP2 mice

3.2.2. Comparison of the mtOxD in cardiac sections, isolated cardiomyocytes and isolated cardiac mitochondria

3.2.3. $E_{\mathrm{GSH}}$ and the mtOxD of cardiac mitochondria isolated from mito Grx1-roGFP2 mice in normoxia versus hypoxia

3.2.4. EGSH and the mtOxD of cardiac mitochondria isolated from mito Grx1-roGFP2 mice during reoxygenation and acute hypoxia 66

3.2.5. Metabolically active mitochondria exhibit a reduction of the mtOxD and $E_{G S H}$

3.2.6. Superoxide anions levels in mitochondria isolated at $20.9 \%$ versus $0.1 \% \mathrm{O}_{2}$ 68

3.2.7. Hydrogen peroxide levels in mitochondria isolated at $20.9 \%$ versus $0.1 \% \mathrm{O}_{2}$ 69

3.2.8. Complex III activity of mitochondria isolated in hypoxia versus normoxia 70

3.2.9. ATP produced by mitochondria isolated in hypoxia versus normoxia 71

3.2.10. Electron transport chain protein complexes are preserved in mouse cardiac mitochondria isolated in normoxia and hypoxia 72

3.2.11. MCU of mouse cardiac mitochondria isolated in normoxia and hypoxia on a Blue Native PAGE ... 73

3.3. Generation of a DAAO-HyPer mouse model

3.3.1. DAAO-HyPer transgenic mice

3.3.2. Confirmation of the DAAO-HyPer transgene by genotyping . 74

3.3.3. Selecting functionally positive founder lines for DAAO-HyPer NES and DAAO-HyPer NLS mice ..... 75

3.4. Epifluorescence microscopic image of the cardiomyocytes isolated from DAAO-HyPer NLS\#9 ...... 76

3.5. Echocardiography of wild type versus transgenic nucleus DAAO-HyPer biosensor mice. 77

3.6. In vitro stimulation of cardiomyocytes isolated from nucleus DAAO-HyPer mouse by D-alanine .. 77

3.7. In vitro stimulation of cardiomyocytes isolated from nucleus DAAO-HyPer mouse by D-alanine versus L-alanine

4. Discussion. 80 
4.1. Ageing affects the redox compartmentalization in cardiomyocytes

4.1.1. Redox compartmentalization in isolated cardiomyocytes from Grx1-roGFP2 mice......................80

4.1.2. EGSH of isolated cardiomyocytes from cyto and mito Grx1-roGFP2 aged mice .............................81

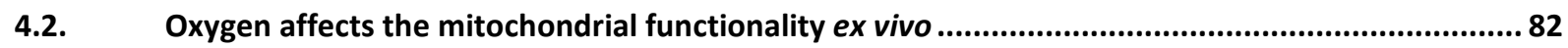

4.2.2. $\quad E_{\mathrm{GSH}}$ of isolated mitochondria in normoxia versus hypoxia..................................................83

4.2.3. $\quad E_{G S H}$ of isolated and energized mitochondria in normoxia versus hypoxia..................................85

4.2.4. ROS levels and complex III enzyme activity in isolated mitochondria isolated in $20.9 \% \mathrm{O}_{2}$ versus $0.1 \%$ O

4.2.5. ATP production by the mitochondria isolated in normoxia versus hypoxia. ...............................87

4.3. Genetically engineered endogenous $\mathrm{H}_{2} \mathrm{O}_{2}$ producer and biosensor: DAAO-HyPer ...................... 89.

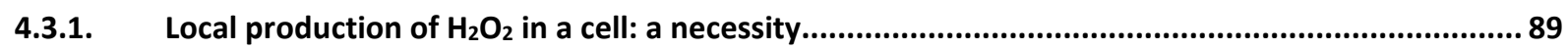

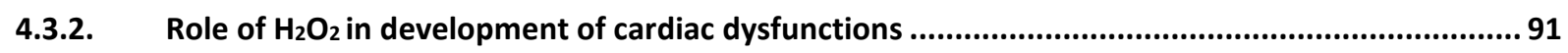

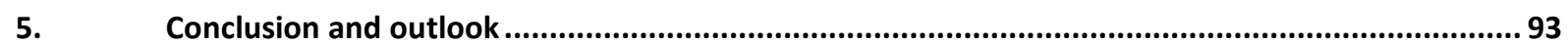

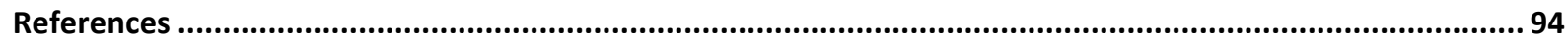

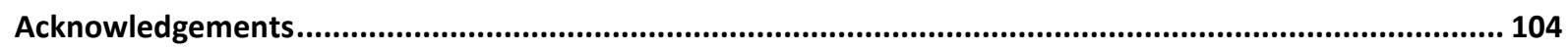




\section{List of figures}

Figure 1: Major sources of Reactive oxygen species (ROS) ........................................11

Figure 2: The glutaredoxin and the thioredoxin antioxidant defense system.....................14

Figure 3: Working mechanism of the Grx1-roGFP2 biosensor.....................................19

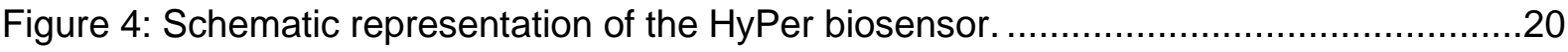

Figure 5: Chemical reaction catalyzed by D-amino acid oxidase (DAAO) . .........................22

Figure 6: Schematic representation of the DAAO-HyPer biosensor..................................24

Figure 7: Schematic representation of a mitochondrial electron transport chain. .................25

Figure 8: Schematic representation of the working mechanism of MCU complex................28

Figure 9: Schematic of the plasmids used for the generation of cyto Grx1-roGFP2 and mito Grx1-roGFP2 transgenic mouse models.

Figure 10: Genotyping results confirming the presence of the transgene in the mito Grx1-

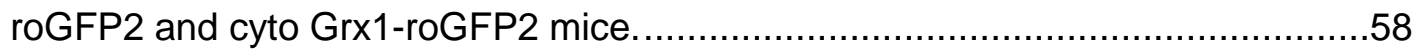

Figure 11: Typical response of Grx1-roGFP cardiomyocytes to $\mathrm{H}_{2} \mathrm{O}_{2}$ and DTT. 59

Figure 12: Grx1-roGFP2 aged mice show significantly poorer heart function than Grx1-roGFP2 young mice.

Figure 13: Redox histology performed with cryosections obtained from a wild type and a mito Grx1-roGFP2 mouse.

Figure 14: The glutathione pool of the cardiac mitochondrial matrix is oxidized during isolation of mitochondria.

Figure 15: Schematic of the experimental setup for isolation of cardiac mitochondria from the mito Grx1-roGFP2 mouse line in normoxia versus hypoxia.

Figure 16: Isolation of mitochondria in hypoxia preserves the reduced $\mathrm{E}_{\mathrm{GSH}}$ and $\mathrm{mtOxD}(\%)$ of the mitochondrial matrix

Figure 17: Exposing isolated mitochondria to normoxia results in an irreversible oxidation of mitochondrial OxD and $E_{\mathrm{GSH}}$.

Figure 18: The mtOxD (\%) of mitochondria isolated and stimulated with succinate in hypoxia is significantly lower as compared to mitochondria isolated in normoxia

Figure 19: Superoxide anions levels are significantly lower in mitochondria isolated in hypoxia as compared to mitochondria isolated in normoxia.

Figure 20: Hydrogen peroxide production in mitochondria isolated at $20.9 \% \mathrm{O}_{2}$ compared to $0.1 \% \mathrm{O}_{2}$. .70

Figure 21: Activity of Complex III is less in mitochondria isolated in hypoxia. .70

Figure 22: Higher amount of ATP is produced by the mitochondria isolated in hypoxia as compared to mitochondria isolated in normoxia. 
Figure 23: Mitochondria isolated in hypoxia and normoxia have similar ETC complex structures detected on Blue Native PAGE.

Figure 24: Mitochondria isolated in hypoxia arrange the $\mathrm{MCU}$ in a high molecular weight supercomplex.

Figure 25: Schematic representation of the plasmids used for generation of cyto DAAO-HyPer and nucleus DAAO-HyPer transgenic mouse models

Figure 26: Genotyping results confirming the presence of the transgene in DAAO-HyPer NES and DAAO-Hyper NLS mice.

Figure 27: DAO-NLS\#9 was found to be the only positive founder line from all the DAAO-NES and the DAAO-NLS mice generated.

Figure 28: Epifluorescence microscopic image of cardiomyocytes expressing DAAO-HyPer

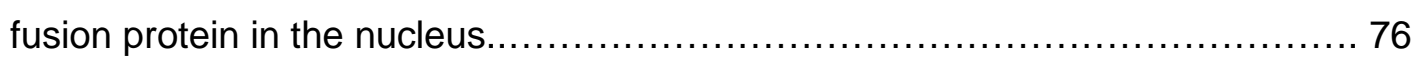

Figure 29: Nucleus DAAO-HyPer transgenic mice have normal heart function. .77

Figure 30: Cardiomyocytes isolated from nucleus DAAO-HyPer mice respond in a dose dependent manner when titrated with various $D$-alanine concentrations. .78

Figure 31: Cardiomyocytes isolated from nucleus DAAO-HyPer mice respond in a dose dependent manner when titrated with various $\mathrm{D}$-alanine concentrations but not to Lalanine.

Figure 32: Schematic representation of the partial oxygen pressure $\left(\mathrm{pO}_{2}\right)$ gradient experienced at sea level to mitochondria present inside a cell. .84

Figure 33: Schematic representation of the observed differences in the components of mitochondria and the mitochondrial ETC when isolated in $20.9 \% \mathrm{O}_{2}$ versus $0.1 \% \mathrm{O}_{2}$ 


\section{List of tables}

Table 1: Examples of enzymatic and non-enzymatic antioxidants. .................................13

Table 2: Examples of chemical sensors to detect ROS ...............................................17

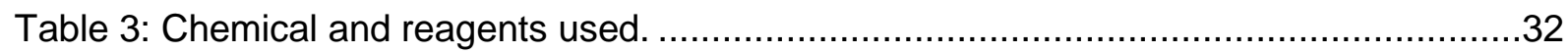

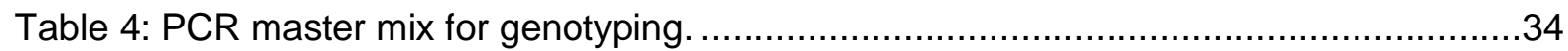

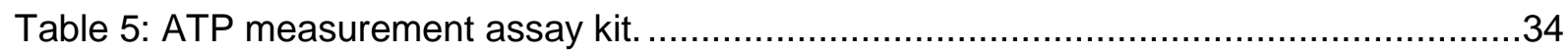

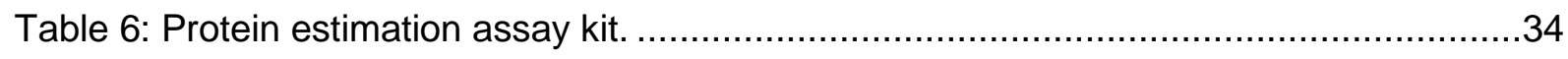

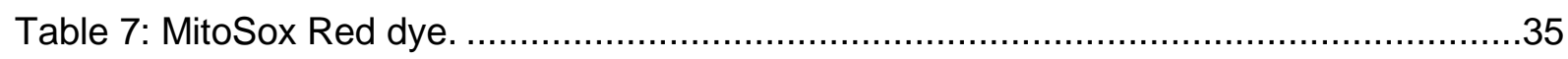

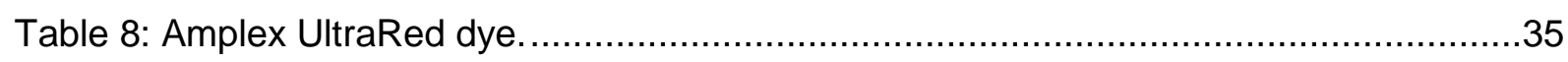

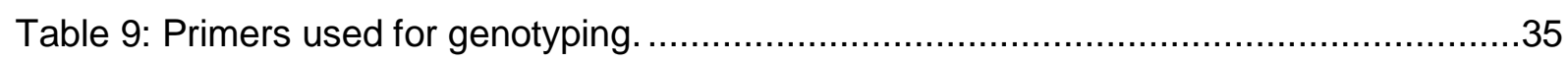

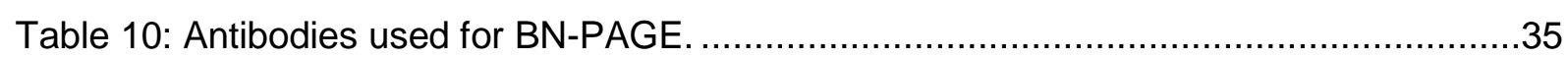

Table 11: Plasmids used for generating transgenic mouse models. ..................................36

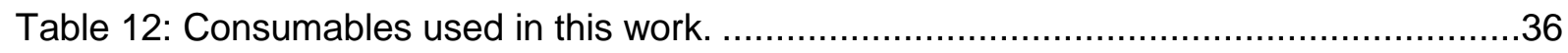

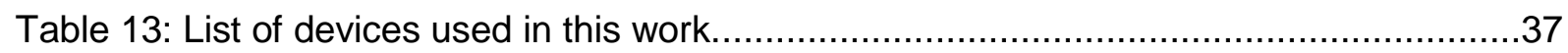

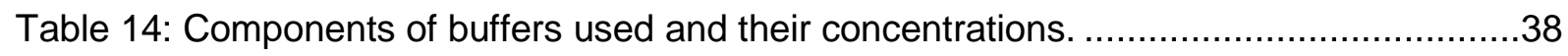

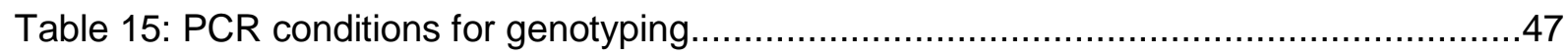

Table 16: Amounts of calcium chloride added during the recalcification step......................48

Table 17: Conditions of each antibody used for detecting the protein of interest...................55

Table 18: Cardiomyocytes exhibit a specific redox compartmentalization in $E_{G S H}$ of the mitochondrial matrix versus the cytoplasm...............................................60

Table 19: Redox compartmentalization between mitochondrial matrix and cytoplasm of the cardiomyocyte tends to disappear with ageing 


\section{Abbreviations}

\begin{tabular}{|c|c|}
\hline${ }^{\circ} \mathrm{C}$ & Degree celcius \\
\hline$\mu g$ & Microgram \\
\hline$\mu l$ & Microliter \\
\hline$\mu \mathrm{M}$ & Micromolar \\
\hline$\cdot \mathrm{O}_{2}^{-}$ & Superoxide anions \\
\hline$\cdot \mathrm{OH}$ & Hydroxyl radicals \\
\hline${ }^{1} \mathrm{O}_{2}$ & Singlet oxygen \\
\hline 2-OH-E+ & 2-hydoxyethidium \\
\hline ADP & Adenosine diphosphate \\
\hline APS & Ammonium persulphate \\
\hline Asn & Aspargine \\
\hline ATP & Adenosine triphosphate \\
\hline ATP & Adenosine triphosphate \\
\hline BDM & 2,3-Butanedione monoxime \\
\hline BN & Blue native \\
\hline bp & Base pair \\
\hline BSA & Bovine serum albumin \\
\hline $\mathrm{CaCl}_{2}$ & Calcium chloride \\
\hline CaMKII & Calmodulin-dependent protein kinase II \\
\hline CoQ & Coenzyme Q \\
\hline $\mathbf{c p}$ & Circularly permutated \\
\hline cTnT & Cardiac troponinT \\
\hline Cys & Cystein \\
\hline DAAO & D-amino acid oxidase \\
\hline DCFDA & Dichlorodihydrofluorescein diacetate \\
\hline DHE & Dihydroethidium \\
\hline DTT & Dithiothreitol \\
\hline $\mathbf{e}^{-}$ & Electron \\
\hline ECC & Excitation-contraction coupling \\
\hline ECL & Enhanced chemiluminescence \\
\hline EDTA & Ethylenediaminetetraacetic acid \\
\hline EF & Ejection fraction \\
\hline$E_{\mathrm{GSH}}$ & Glutathione potential \\
\hline EMRE & Essential MCU regulator \\
\hline
\end{tabular}




\begin{tabular}{|c|c|}
\hline ETC & Electron transport chain \\
\hline EtOH & Ethanol \\
\hline FAS & Fractional area shortening \\
\hline $\mathrm{Fe}-\mathrm{S}$ & Iron-sulfur clusters \\
\hline FMN & Flavin mononucleotide \\
\hline g & Gram \\
\hline GFP & Green fluorescent protein \\
\hline Gln & Glutamine \\
\hline GPx & GSH peroxidase \\
\hline GR & Glutathione reductase \\
\hline Grx & Glutaredoxin \\
\hline GS & Glutathione synthetase \\
\hline GSH & Glutathione \\
\hline GSSG & Oxidized GSH \\
\hline $\mathrm{H}_{2} \mathrm{O}$ & Water \\
\hline $\mathrm{H}_{2} \mathrm{O}_{2}$ & Hydrogen peroxide \\
\hline $\mathrm{HCl}$ & Hydrochloric acid \\
\hline HDAC & Histone deacetylases \\
\hline HE & Hydroethidium \\
\hline $\mathrm{Hg}$ & Mercury \\
\hline $\mathrm{HOCl}$ & Hypochlorous acid \\
\hline HRP & Horseradish peroxidase \\
\hline IMS & Mitochondrial intermembrane space \\
\hline KCL & Potassium chloride \\
\hline $\mathrm{KH}_{2} \mathrm{PO}_{4}$ & Monopotassium phosphate \\
\hline $\mathrm{KHCO}_{3}$ & Potassium bicarbonate \\
\hline $\mathrm{mA}$ & Mili ampere \\
\hline MCU & Mitochondrial calcium uniporter \\
\hline MEF2 & Myocyte enhancer factor 2 \\
\hline $\mathrm{MgSO}_{4}$ & Magnesium sulfate \\
\hline $\min$ & Minutes \\
\hline ml & Mililiter \\
\hline $\mathrm{mM}$ & Milimolar \\
\hline $\mathbf{m m}$ & Milimeter \\
\hline molar & Molar \\
\hline MOPS & 3-(N-Morpholino)propanesulfonic acid \\
\hline ms & Miliseconds \\
\hline
\end{tabular}




\begin{tabular}{|c|c|}
\hline mtOXD & Mitochindrial oxidation difference \\
\hline $\mathbf{m V}$ & Milivolt \\
\hline $\mathrm{Na}_{2} \mathrm{HPO}_{4}$ & Disodium phosphate \\
\hline $\mathrm{NaCl}$ & Sodium chloride \\
\hline NADH & Nicotinamide adenine dinucleotide \\
\hline NADPH & Nicotinamide adenine dinucleotide phosphate \\
\hline $\mathrm{NaHCO}_{3}$ & Sodium bicarbonate \\
\hline NAOH & Sodium hydroxide \\
\hline NEM & $\mathrm{N}$-Ethylmaleimide \\
\hline NES & Nuclear export signal \\
\hline NFAT & Nuclear factor of activated $\mathrm{T}$ cell \\
\hline NLS & Nuclear localization signal \\
\hline nm & Nanometer \\
\hline $\mathrm{nM}$ & Nanomolar \\
\hline $\mathrm{nm}$ & Nanometer \\
\hline NNT & Nicotinamide nucleotide transhydrogenase \\
\hline NO & Nitric oxide \\
\hline NOX & NADPH oxidases \\
\hline $\mathrm{O}_{2}$ & Oxygen \\
\hline OxD & Oxidation difference \\
\hline PAGE & Polyacrylamide gel electrophoresis \\
\hline PCR & Ploymerase chain reaction \\
\hline PFA & Paraformaldehyde \\
\hline PKA & Protein kinase $A$ \\
\hline PKG & Protein kinase $\mathrm{G}$ \\
\hline pmol & Picomolar \\
\hline PMSF & Phenylmethylsulfonyl fluoride \\
\hline $\mathrm{pO}_{2}$ & Partial oxygen pressure \\
\hline Prx & Peroxiredoxin \\
\hline PVDF & Polyvinylidene difluoride \\
\hline RET & Reverse electron transport \\
\hline ro & Reduction-oxidation sensitive \\
\hline RO• & Alkoxyl radicals \\
\hline ROO' & Peroxyl radicals \\
\hline ROS & Reactive oxygen species \\
\hline rpm & Rotations per minute \\
\hline rXYFP & Redox sensitive YFP \\
\hline
\end{tabular}




$\begin{array}{ll}\text { S } & \text { Seconds } \\ \text { SDS } & \text { Polyvinylidene difluoride } \\ \text { SEM } & \text { Standard error of mean } \\ \text { Ser } & \text { Serine } \\ \text { SOD } & \text { Superoxide dismutase } \\ \text { TEMED } & \text { Tetramethylethylenediamine } \\ \text { Thr } & \text { Threonine } \\ \text { TPP } & \text { Triphenylphosphonium } \\ \text { TrX } & \text { Thioredoxin } \\ \text { V } & \text { Voltage } \\ \text { wt } & \text { Wild type } \\ \text { YFP } & \text { Yellow fluorescent protein } \\ \boldsymbol{\alpha}-\text { MHC } & \text { Alpha-myosine heavy chain } \\ \text { Y-GS } & \text { Y-glutamylcysteine }\end{array}$




\section{Abstract}

Reactive oxygen species (ROS) are highly reactive molecules produced in any biological system. When ROS are produced in higher amounts, they are lethal to cells. Therefore cells possess a tight redox regulation through action of various antioxidant defense systems. An imbalance between the ROS produced versus the action of the antioxidants can give rise to a state called oxidative stress. In order to study the consequences of high ROS production in a system, it is essential to develop tools that can measure quantitatively the precise levels of specific ROS or the status of a specific redox couple. Until recently, synthetic probes were used widely to measure ROS in a qualitative manner. However, taking into consideration the limitations of these probes, genetically encoded biosensors have gradually started to replace the relatively non-specific probes. These genetically encoded biosensors can not only visualize the redox nature quantitatively and in real time but also can be targeted to any subcellular compartment of a cell. In line to these necessities, mouse models in which the glutathione redox biosensor Grx1-roGFP2 is expressed in cardiomyocytes and located in two different compartments were applied in the presented thesis. These mouse models allow to study the glutathione redox potential $\left(E_{\mathrm{GSH}}\right)$ in the cytoplasm versus mitochondrial matrix. The mouse models were used to study the effect of aging on the $E_{\mathrm{GSH}}$ of the cytoplasm and mitochondrial matrix in cardiomyocytes. The redox compartmentalization between the two compartments which was observed in young mice seems to disappear in aging animals. Besides applying the mouse models to study the effects of aging on redox regulation, the mito Grx1-roGFP2 mouse model was utilized to study the importance of maintaining the physiological oxygen concentration in order to preserve the reduced $E_{\mathrm{GSH}}$ of the mitochondrial matrix as well as the overall mitochondrial functionality. In this part of the thesis, it was observed that upon isolation of mitochondria from cardiac tissue at room air conditions $\left(20 \% \mathrm{O}_{2}\right)$, mitochondria seem to almost fully get oxidized However, when the mitochondria are isolated in hypoxia $\left(0.1 \% \mathrm{O}_{2}\right)$, the $E_{\mathrm{GSH}}$ is preserved demonstrating that the $E_{\mathrm{GSH}}$ of the mitochondrial matrix is indeed affected by the change in the $\mathrm{pO}_{2}$ experienced by the mitochondria when isolated from the tissue. Together with the $E_{\mathrm{GSH}}$, other parameters of the mitochondrial electron transport chain like ROS, ATP as well as complex III activity are affected when mitochondria are isolated in $20 \%$ $\mathrm{O}_{2}$. In the final part of my thesis, I generated a novel redox biosensor mouse model. In these mice the biosensor consists of the endogenous $\mathrm{H}_{2} \mathrm{O}_{2}$ producer D-amino acid oxidase (DAAO) fused with the $\mathrm{H}_{2} \mathrm{O}_{2} \mathrm{HyPer}$ biosensor. A positive founder line of the DAAO-HyPer, wherein the biosensor is targeted to the nucleus of the cardiomyocytes, was successfully created and characterized. This mouse model is useful to study the development of cardiac dysfunctions 
in consequence to the generation of the endogenous ROS in the nucleus of the cardiomyocytes. 


\section{Introduction}

Aerobic organisms use molecular oxygen $\left(\mathrm{O}_{2}\right)$ to generate chemical energy in the form of adenine triphosphate (ATP). The thermodynamic properties of $\mathrm{O}_{2}$ are the basis for serving as the terminal electron acceptor in the reduction of carbon-based fuels to generate ATP by oxidative phosphorylation. As a by-product of $\mathrm{O}_{2}$ consumption, reactive oxygen species (ROS) are produced. In line, ROS appeared about 2.4-3.8 billion years ago together with the evolution of aerobic life. Ever since, highly sophisticated cellular ROS producer and scavenging systems have developed. Therefore ROS are regarded on the one hand as toxic by-products of aerobic life. On the other hand, it is quite obvious that they also evolved to regulate cellular processes such as differentiation, stress signaling, systemic responses, and cell death among others in plants, animals, and most eukaryotic organisms. To this end, the fine-tuning of ROS production and scavenging is essential for proper cellular function.

\subsection{Reactive oxygen species and their sources}

ROS are the derivatives of $\mathrm{O}_{2}$ produced in any biological system. These derivatives can be categorized into two different groups, i.e. free radicals or non-radical derivatives of oxygen (Halliwell, 2015; Phaniendra, Jestadi, \& Periyasamy, 2015). A free radical is defined as an atom or molecule containing one or more unpaired electrons in the valency shell or outer orbit. The uneven number of electrons makes it unstable, short lived and highly reactive. The free oxygen-centered radicals include superoxide anions $\left({ }^{\circ} \mathrm{O}_{2}{ }^{-}\right)$, hydroxyl radicals $\left({ }^{\circ} \mathrm{OH}\right)$, alkoxyl radicals (RO') and peroxyl radicals (ROO'). Non-radical derivatives are either oxidizing agents or are easily converted into radicals, such as hypochlorous acid $(\mathrm{HOCl})$, singlet oxygen $\left({ }^{1} \mathrm{O}_{2}\right)$, and hydrogen peroxide $\left(\mathrm{H}_{2} \mathrm{O}_{2}\right) . \mathrm{H}_{2} \mathrm{O}_{2}$ is a very important cellular oxidant since it can cross biological membranes and form the highly reactive ${ }^{\circ} \mathrm{OH}$ by interaction with transition metal ions such as $\mathrm{Fe}^{2+}$ or $\mathrm{Cu}^{+}$.

ROS can be produced from either endogenous or exogenous sources. Endogenous cellular sources are associated to organelles with high oxygen turnover including mitochondria, peroxisomes and the endoplasmic reticulum. There are various ROS producing enzymes present in the peroxisomes such as Acyl CoA-oxidase, D-amino acid oxidase, L-a-hydroxyoxidase, xanthine oxidase (De Duve and Baudhuin, 2017). The enzymes present in the endoplasmic reticulum responsible for ROS production include cytochrome P450, b5 enzymes and diamine oxidase. Other endogenous ROS producing events include for example prostaglandin synthesis, auto-oxidation of adrenaline and immune cell activation. The major cellular producers of endogenous ROS are the NADPH oxidases (NOX) and components of the electron transport chain (ETC) in the mitochondria (Fig. 1). 


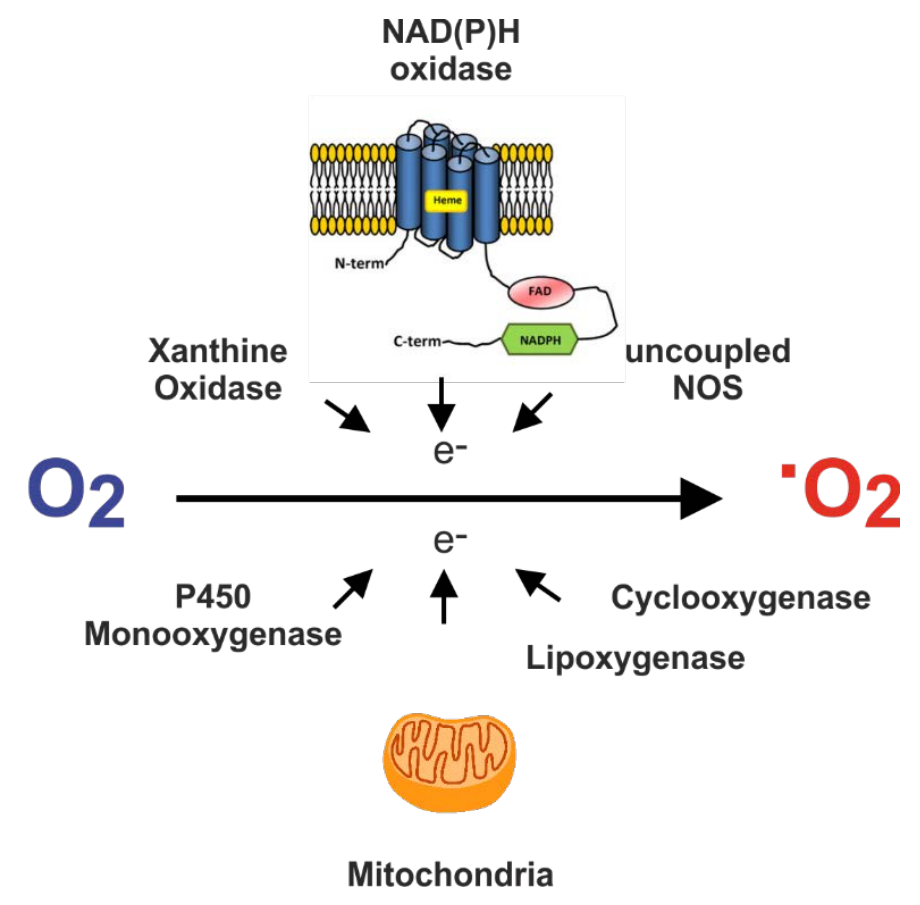

Figure 1: Major sources of Reactive oxygen species (ROS). Molecular oxygen accepts the electron from various sources like the electron transport chain in the mitochondria, NADPH oxidase, xanthine oxidase, monooxygenase, lipoxygenase, cyclooxygenase and gets converted to superoxide anion $\left({ }^{\circ} \mathrm{O}_{2}{ }^{-}\right)$.

\subsubsection{Mitochondrial ROS}

The majority of oxygen used in a cell is expended in the mitochondria. Generation of mitochondrial ROS mainly takes place at the ETC located on the inner mitochondrial membrane during oxidative phosphorylation (Gambardella et al., 2017). Along with the production of ATP, the ETC produces ${ }^{\circ} \mathrm{O}_{2}{ }^{-}$. Initially, it was thought that mitochondria produce ROS in the form of $\mathrm{H}_{2} \mathrm{O}_{2}$ (Loschen et al., 1971). However, with time it was discovered that mitochondrial ROS is primarily produced in the form of ${ }^{\circ} \mathrm{O}_{2}{ }^{-}$, which subsequently undergoes dismutation by the superoxide dismutase (SOD) to form $\mathrm{H}_{2} \mathrm{O}_{2}$. There are several reports about the production of $\mathrm{H}_{2} \mathrm{O}_{2}$ in isolated mitochondria which in turn would give more information on the production of ${ }^{\circ} \mathrm{O}_{2}{ }^{-}$from the mitochondria. There are two main modes because of which there is huge efflux of $\mathrm{H}_{2} \mathrm{O}_{2}$ from isolated mitochondria (Murphy, 2008). The first mode directs towards the high $\mathrm{NADH} / \mathrm{NAD}^{+}$ratio in the mitochondrial matrix leading to a high flow of electrons, which eventually can generate a high $\mathrm{H}_{2} \mathrm{O}_{2}$ efflux (Kudin et al., 2004). Highly reduced coenzyme $\mathrm{Q}$ (CoQ) along with a combination of high proton motive force and no ATP production, contributes to the second mode of $\mathrm{H}_{2} \mathrm{O}_{2}$ efflux (Korshunov et al., 1997).

In the ETC, complex I and complex III are major sites of ${ }^{\circ} \mathrm{O}_{2}^{-}$production (Murphy, 2008). In complex I, the electron flow is initiated when the $\mathrm{NADH}$ is reduced to $\mathrm{NAD}^{+}$. Electrons are transferred from $\mathrm{NADH}$ to the Flavin mononucleotide (FMN) cofactor and further to CoQ via 7 
iron-sulfur clusters (Fe-S) (Fearnley et al., 2003). There is a high tendency of electron leakage while the electrons are passing from FMN to CoQ via Fe-S (Kudin et al., 2004). In case of lower ATP demands, the respiration rate also decreases giving rise to higher NADH/ NAD ${ }^{+}$ ratio. The higher the $\mathrm{NADH}$, the higher is the proportion of the reduced FMN. As a consequence of a highly reduced FMN and a lower ATP demand, there is the possibility of oxygen accepting the leaky electron and getting converted to $\mathrm{O}_{2}{ }^{-}$. The other known mechanism for the production of ${ }^{\circ} \mathrm{O}_{2}{ }^{-}$from complex $\mathrm{I}$ is the reverse electron transport (RET) (Esterházy et al., 2008). RET takes place when CoQ is extremely reduced by the electrons, however, there is a high proton motive force which drives the electrons back to FMN. Reduced FMN is an active site for the production of ${ }^{\circ} \mathrm{O}_{2}{ }^{-}$from complex I to the elevated $\mathrm{NADH} / \mathrm{NAD}^{+}$ ratio.

Complex III passes the electrons from CoQ to cytochrome c through the Q-cycle (Kudin et al., 2004; leier and Dröse, 2013). Experimentally, in presence of CoQH2 when the $\mathrm{Q}_{i}$ site was inhibited by antimycin, a large amount of ${ }^{\circ} \mathrm{O}_{2}{ }^{-}$was produced from the oxygen reacting with ubisemiquinone in the $Q_{0}$ site. On the other hand, it is also observed that when the 'distal' $Q_{0}$ site is inhibited by an inhibitor like stigmatellin, there is a complete blockage of ${ }^{\circ} \mathrm{O}_{2}{ }^{-}$production from complex III (Muller et al., 2003). However, when the 'proximal' Qo site is inhibited by inhibitors like myxothiazol, there is still a small amount of ${ }^{\circ} \mathrm{O}_{2}{ }^{-}$from complex III. Rottenberg and colleagues also showed the importance of applied membrane potential on the formation of ${ }^{\circ} \mathrm{O}_{2}{ }^{-}$ (Rottenberg et al., 2009). As higher the membrane potential is as higher is the ${ }^{\circ} \mathrm{O}_{2}{ }^{-}$production. The membrane potential slows down the electron transfer from heme $b_{\llcorner}$to heme $b_{H}$, which is the only one transmembrane electrogenic step in the Q-cycle. This makes the heme $b_{\llcorner}$more reduced, resulting into more ${ }^{\circ} \mathrm{O}_{2}^{-}$production.

\subsubsection{NADPH oxidase}

NOX are transmembrane proteins that can transport electrons across the membrane to reduce oxygen to ${ }^{\circ} \mathrm{O}_{2}{ }^{-}$(Bedard and Krause, 2007). The family of NOX enzymes consists of 7 catalytic subunits namely Nox1-5, Duox 1-2 and their regulatory subunits such as p22phox, p47phox or Noxo1, p67phox or Noxa1 and p40phox (Altenhöfer et al., 2012). NOX function was first analyzed mainly in neutrophils and macrophages in the context of the respiratory burst (Nauseef, 2017). This led to the discovery of Nox2. Upon activation in innate immune cells, Nox2 converts molecular oxygen to ${ }^{\circ} \mathrm{O}_{2}{ }^{-}$at the expense of $\mathrm{NADPH}$. These ${ }^{\circ} \mathrm{O}_{2}{ }^{-}$are then used to combat the invading micro-organisms that initiated the respiratory burst. Other than being a catalyst in respiratory burst, NOXs are also involved in specific signaling pathways. Nox 2 and 4 are highly expressed in cardiovascular tissues (Lassègue et al., 2012; Lambeth, 2004). 


\subsection{Antioxidant defense systems}

Antioxidants are compounds that help to compensate and balance the effects of ROS produced inside a cell. Antioxidant defense systems comprise enzymatic and non-enzymatic antioxidants (Table 1).

\begin{tabular}{|l|l|}
\hline Enzymatic & Non-enzymatic \\
\hline Catalase & Ascorbic acid (Vitamin C) \\
\hline Glutathione Peroxidase & Glutathione (GSH) \\
\hline Thioredoxin (Trx) & Uric acid \\
\hline Peroxiredoxin (Prx) & $\alpha$-Tocopherol (Vitamin E) \\
\hline Superoxide dismutase (SOD) & $\beta$-Carotene \\
\hline
\end{tabular}

Table 1: Examples of enzymatic and non-enzymatic antioxidants (adapted from Hafstad et al., 2013).

The two major redox systems that play a vital role as an antioxidant defense system in a cell are the glutaredoxin and the thioredoxin system (Fig. 2).

Glutathione (GSH) is one of the major mediators and regulators of cellular redox processes. It is one of the most abundant (0.1-10 mM) low molecular weight peptides found in mammalian cells (Meister, 1988). GSH is synthesized in the cytoplasm of a cell by sequential reactions at an expense of ATP (White et al., 2003; Forman et al., 2009). It is a tripeptide containing glutamic acid, cysteine and glycine. The first rate-limiting enzyme for the synthesis of GSH is the $\mathrm{Y}$-glutamylcysteine synthetase. The second rate-limiting step includes the conversion of $\mathrm{Y}^{-}$ glutamylcysteine ( $\mathrm{Y}-\mathrm{GS}$ ) to $\mathrm{GSH}$ by glutathione synthetase (GS).

GSH is present in a cell as a reduced form (GSH) and an oxidized form (GSSG) or an oxidized thiol protein with glutathione (GS-R). Especially the cysteine residue of the GSH is known to be an important player in ROS scavenging (Espinosa-Diez et al., 2015; Ray et al., 2012). GSH works as an antioxidant buffer where GSH by itself gets converted to its oxidized form (Aoyama and Nakaki, 2015). The enzyme GSH peroxidase (GPx) oxidizes the $\mathrm{GSH}$, reducing the $\mathrm{H}_{2} \mathrm{O}_{2}$. The GSSG can revert back to its reduced form at the expense of an electron from NADPH with the help of the enzyme Glutathione reductase (GR). Other than buffering $\mathrm{H}_{2} \mathrm{O}_{2}, \mathrm{GSH}$ also has the capacity to rescue oxidized proteins. The cysteine residue of any surrounding oxidized protein can form a disulfide bond with GSH in a mechanism called S-glutathionylation, leaving the protein in its reduced form. This mechanism is reversible through the enzyme glutaredoxin (Grx). Taken together, GSH serves as an important redox buffer for maintaining the redox status. 
Thioredoxin (Trx) is a small protein oxidoreductase enzyme containing a dithiol-disulfide active site (Snoep et al., 2008). The primary function of Trx is the reduction of oxidized cysteine residues and the cleavage of disulfide bonds. The reduced $\operatorname{Trx}\left(\operatorname{Trx}(\mathrm{SH})_{2}\right)$ gets itself oxidized, by reducing the oxidized target protein. Thioredoxin reductase (TR) catalyzes the reversal of the oxidized $\operatorname{Trx}(\operatorname{Trx}(\mathrm{SS}))$ to reduced $\operatorname{Trx}\left(\operatorname{Trx}(\mathrm{SH})_{2}\right)$ with NADPH as a source of an electron.

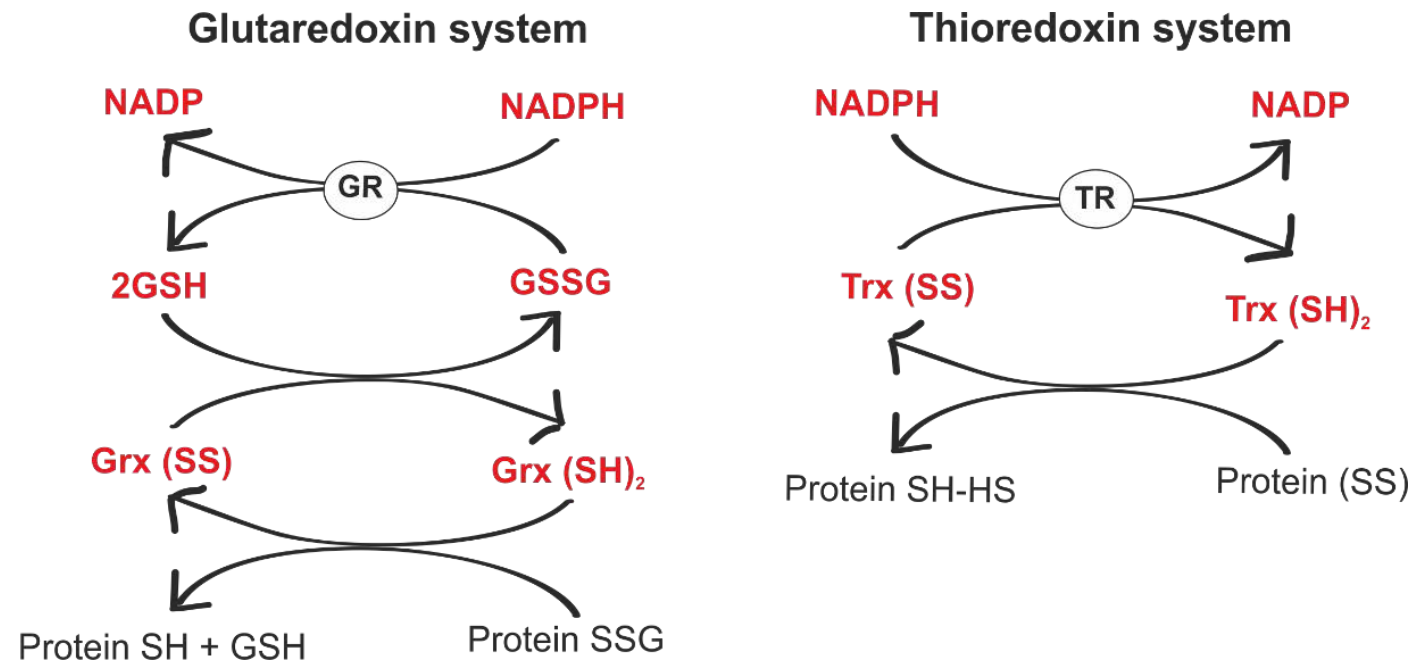

Figure 2: The glutaredoxin and the thioredoxin antioxidant defense system. Oxidized glutaredoxin (Grx) is reduced by $2 \mathrm{GSH}$. GSSG is reduced back to GSH by the enzyme glutathione reductase (GR) at an expense of electron from NADPH. Oxidized thioredoxin (Trx) is reduced back by thioredoxin reductase (TR) with NADPH as an electron donor.

\subsection{Oxidative stress}

Oxidative stress occurs when the production of ROS exceeds the defense provided by the antioxidants (Dröge, 2015). Balanced levels of ROS are essential for proper cell function and homeostasis. However, a larger amount of ROS produced than required can prove to be fatal to a cell (Liguori et al., 2018). In line, oxidative stress is associated to various diseases like aging, diabetes, cancer, chronic kidney diseases, neurodegenerative diseases and cardiovascular diseases.

Ageing is a process where the functions of all organs gradually begin to slow down leading to the occurrence of diseases and finally death (Birch-Machin and Bowman, 2016). Ageing is a naturally occurring process in any living organism. However, this process can be accelerated by occasions like oxidative stress. One of the original theories of ageing suggests that ROS are a part of the ageing process due to their reactive nature to cellular molecules, especially in mitochondria. There are various studies demonstrating different theories about the relation between antioxidants and ageing. Early studies proposed that antioxidants like SOD or catalase in the cytosol can increase the life span in Drosophila (Orr and Sohal, 1994). On the other hand in mice, overexpression of SOD with catalase in the cytosol and only SOD in 
mitochondrial matrix does not indicate any longevity (Pérez et al., 2009). However, overexpression of catalase in the mitochondrial matrix but not the cytoplasm or nucleus does show longevity in mice (Schriner et al., 2009). Altogether, this indicates that compartment and ROS-specific effects need to be analyzed in more detail.

\subsection{Detection of ROS}

Because of the importance of ROS for cellular physiology and pathophysiology, there is plenty of research on how to detect ROS. Various methods on measuring ROS in vitro from any biological sample have been developed. These methods can be broadly classified into two categories, i.e. dye-based detection with synthetic probes and detection by the use of genetically engineered biosensors.

\subsubsection{Synthetic probes}

Synthetic probes are widely employed for the detection of ROS making use of intensity-based fluorescence measurements. These fluorescence probes are very sensitive and easy to use, but lack in part specificity and can produce artifacts. The most frequently used synthetic probes are nitroblue tetrazolium, dihydroethidium, MitoSOX, amplex red, cytochrome c and dichlorodihydrofluorescein diacetate (Griendling et al., 2016). Table 2 summarizes the ROS detected along with the advantages and disadvantages of using these probes.

\section{Nitroblue tetrazolium}

Nitroblue tetrazolium is used to detect ${ }^{\circ} \mathrm{O}_{2}{ }^{-}$(Hyung et al., 2006). The interaction of ${ }^{\circ} \mathrm{O}_{2}{ }^{-}$with the water-soluble yellow nitroblue tetrazolium results in the conversion to a blue formazan. This assay can give a qualitative insight on the basis of absorbance of the blue color measured at $620 \mathrm{~nm}$.

\section{Dihydroethidium}

Dihydroethidium based assays work on the principle of the extent of oxidation of $\mathrm{DHE}$ by ${ }^{\circ} \mathrm{O}_{2}{ }^{-}$ to 2-hydoxyethidium (2-OH-E+) (Zielonka and Kalyanaraman, 2010). However, this fluorescent probe is not very specific since it can also be oxidized by other factors like $\mathrm{ONOO}^{-}, \mathrm{OH}^{\circ} \mathrm{H}_{2} \mathrm{O}_{2}$ along with ${ }^{\circ} \mathrm{O}_{2}^{-}$. In addition, DHE can also be oxidized just because of a change in the atmospheric oxygen or even by light. This makes DHE non-specific as a ROS marker.

\section{MitoSOX}

MitoSOX, also known as hydroethidium (HE) is a mitochondrial ${ }^{\circ} \mathrm{O}_{2}{ }^{-}$detection dye (Murphy et al., 2006). It comprises a triphenylphosphonium $\left(\mathrm{TPP}^{+}\right)$group which allows the entry of 
lipophylic phenyl groups attached to it to go across the phospholipid bilayer of mitochondria. In this way, the MitoSOX dye can get gathered in the mitochondrial matrix. ${ }^{\circ} \mathrm{O}_{2}{ }^{-}$convert $\mathrm{HE}$ into a hydroxylated product $\left(\mathrm{HO}-\mathrm{Etd}^{+}\right)$which can be detected at an excitation wavelength of 396 $\mathrm{nm}$.

\section{Amplex red}

Amplex red is a non-florescent dye, which can get oxidized to the fluorescent product resorufin by $\mathrm{H}_{2} \mathrm{O}_{2}$ in the presence of horseradish peroxidase (HRP) (Zhou et al., 1997). The fluorescent product can be detected at an excitation wavelength of $535 \mathrm{~nm}$ and an emission wavelength of $590 \mathrm{~nm}$. In order to rule out nonspecific oxidation by some other radicals than $\mathrm{H}_{2} \mathrm{O}_{2}$, superoxide dismutase (SOD) is usually used during the assay (Summers et al., 2013).

\section{Cytochrome c reduction}

Cytochrome $\mathrm{c}$ reduction assay works on the principle of ferricytochrome $\mathrm{c}$ getting oxidized to ferrocytochrome $\mathrm{c}$ on accepting an electron from ${ }^{\circ} \mathrm{O}_{2}{ }^{-}$(Vandewalle and Petersen, 1987). This oxidation can be detected at an absorbance of $550 \mathrm{~nm}$. However, the cytochrome c can also get oxidized by $\mathrm{H}_{2} \mathrm{O}_{2}$. This can affect the absorbance intensities measured at $550 \mathrm{~nm}$.

\section{Dichlorodihydrofluorescein}

Dichlorodihydrofluorescein Diacetate (DCFDA/ $\mathrm{H}_{2} \mathrm{DCFDA}$ ) once diffused into a cell is deacetylated by cellular esterases to $\mathrm{H}_{2} \mathrm{DCF}$ (Tetz et al., 2013). Further on $\mathrm{H}_{2} \mathrm{DCF}$ is oxidized by ROS to form dichlorofluorescein (DCF). This fluorescent compound can be measured spectroscopically at $495 \mathrm{~nm}$ with an emission of light at $529 \mathrm{~nm}$.

\begin{tabular}{|l|l|l|l|}
\hline Probe & Species detected & Advantages & Disadvantages \\
\hline Nitroblue tetrazolium & $\mathrm{O}_{2}-$ & $\begin{array}{l}\text { Simple and most } \\
\text { widely used test }\end{array}$ & Low sensitivity \\
\hline Dihydroethidium & $\mathrm{O}_{2}-$ & $\begin{array}{l}\text { Simple plate reader } \\
\text { assay widely used } \\
\text { for mitochondrial } \\
\text { ROS detection }\end{array}$ & $\begin{array}{l}\text { Non-specific, } \\
\text { oxidation by other } \\
\text { radicals }\end{array}$ \\
\hline MitoSoX & $\mathrm{O}_{2}-$ & $\begin{array}{l}\text { Easy, quick and } \\
\text { inexpensive }\end{array}$ & $\begin{array}{l}\text { Sensitive dye, need } \\
\text { to handle with care }\end{array}$ \\
\hline
\end{tabular}




\begin{tabular}{|l|l|l|l|}
\hline Amplex Red & $\mathrm{H}_{2} \mathrm{O}_{2}$ & $\begin{array}{l}\text { Low background, } \\
\text { High sensitivity, } \\
\text { Robustness }\end{array}$ & $\begin{array}{l}\text { Peroxidase- } \\
\text { dependent } \\
\text { fluorescence } \\
\text { interference by } \\
\text { NADPH in cell-free } \\
\text { assays }\end{array}$ \\
\hline Cytochrome c & $\mathrm{O}_{2-}$ & $\begin{array}{l}\text { Simple plate reader } \\
\text { assay }\end{array}$ & Low sensitivity \\
\hline $\begin{array}{l}\text { Dichlorodihydrofluor- } \\
\text { escein derivatives }\end{array}$ & General ROS probe & $\begin{array}{l}\text { Cell-permeable and } \\
\text { highly fluorescent }\end{array}$ & $\begin{array}{l}\text { Nonselective, Auto } \\
\text { oxidation }\end{array}$ \\
\hline
\end{tabular}

Table 2: Examples of chemical sensors to detect ROS (adapted from Griendling et al., 2016).

\subsubsection{Genetically engineered redox biosensors}

As seen in the earlier section, ROS can be detected by various chemical sensors. However, chemical sensors possess several disadvantages with regards to specificity and sensitivity. Through recent progress, there are genetically engineered redox biosensors established. The use of these biosensors has directed a novel path to detect ROS in a quantitative, specific and highly sensitive manner. Following are few examples of the most commonly used redox biosensors given:

\section{GSH based redox biosensors}

GSH is an important mediator in redox processes. It is very crucial for a cell to maintain a fine balance between 2 GSH/GSSG. It is equally interesting to detect the 2GSH/GSSG ratio present inside a cell during a particular stage of cellular processes and metabolism, where the role of GSH based redox biosensors materializes. GSH based biosensors consist of a pair of cysteine residues attached to the chromophore. The redox state of the pair of cysteine residues depends on the GSH pool in the surrounding of the biosensor. Due to the changes in the redox state of the cysteine residues of the chromophore a conformational change occurs in the protein. This conformational change will alter the chromophore's excitation spectra which can be recorded and quantitatively analyzed. Based on this theory, there are several derivatives of GSH based biosensors developed that can detect the 2 GSH/GSSG ratio. Few of the examples are stated as follows: 


\section{rXYFP}

The first biosensor generated in the past to detect the 2GSH/GSSG ratio is a mutated yellow fluorescent protein containing the mutations Asn149Cys and Ser202Cys (Østergaard et al., 2001). This biosensor was named redox sensitive YFP (rxYFP). During oxidation, there is a formation of a disulfide bond between the Cys149 and Cys202 which results in a 2-fold decrease of the excited intensity measured at $512 \mathrm{~nm}$. This change can be recorded in order to quantify the extent of oxidation. YFP has 2 excitation wavelengths, one at the neutral Aband (392 nm) and the other at the anionic B-band (514 nm) (Wachter et al., 2000). However, the neutral band is non-fluorescent due to fluorescence quenching. Due to this reason, there is only one excitation peak functional for rxYFP at $512 \mathrm{~nm}$. On the contrary, the use of rxYFP sensor is also shown to be ratiometric at two excitation wavelengths of 458 and $488 \mathrm{~nm}$ (Winther et al., 2008). Since the excitation wavelength of $458 \mathrm{~nm}$ is very close to the isosbestic point of YFP, the fluorescence changes at this wavelength however are very weak. This can have a huge impact on background noise correction which already makes this sensor a difficult probe to handle. Not only this but also the reactivity of rxYFP with GSH is slow. This makes it time wise critical to plan an experiment using this sensor taking into account air oxidation of GSH which can possibly lead to non-specific readings.

\section{Reduction-oxidation sensitive Green fluorescent protein (roGFP)}

roGFP is one of the derivatives of the green fluorescent protein (GFP) which is engineered to be sensitive to redox changes. It can be utilized for real time visualization of the changes in the excitation spectra during oxidation at 400 and $490 \mathrm{~nm}$. roGFP1 was one of the first biosensors developed after rxYFP (Hanson et al., 2004; Dooley et al., 2004). roGFP1 harbors a replacement at Ser147Cys and GIn204Cys along with a mutation at Cys48Ser compared to the wtGFP. There is another derivative generated called roGFP2 which contains a mutation at Ser65Thr of the roGFP1 in addition (Dooley et al., 2004). Upon oxidation of roGFP2, there is an increase in the fluorescence intensity at excitation wavelength of $405 \mathrm{~nm}$ and a decrease at excitation wavelength of $488 \mathrm{~nm}$ whereas an opposite change is observed upon reduction. In the case of roGFP1, a decrease is observed in the $400 \mathrm{~nm}$ wavelength upon oxidation (Lukyanov and Belousov, 2014; Hanson et al., 2004). This decrease results into the weakening of a weak signal together with an increase observed in the brighter signal at $490 \mathrm{~nm}$. This combination of detecting a weaker signal from an already weak signal and a much brighter signal from an initially bright signal is inconvenient for imaging. In contrast, an opposite scenario is observed in the case of roGFP2 which makes it easier for detection. For these reasons, roGFP2 proved to be more dynamic and useful. 


\section{Grx1-roGFP2}

roGFP2 proved to be a useful biosensor but, it also has some drawbacks. The disulfide bridge formation between the two cysteine residues is a slow reaction. The reaction can be catalyzed by the enzyme Grx (Meyer et al., 2007). To this end Grx1 was fused to roGFP2, which makes the sensor more sensitive and faster. In line, this upgraded roGFP2 biosensor was named Grx1-roGFP2. It can detect the specific GSH redox potential $\left(E_{\mathrm{GSH}}\right)$ in the range of -240 to $320 \mathrm{mV}$, making it very sensitive even at very low concentrations of GSH/GSSG (Bilan et al., 2015).

The presence of an oxidized environment eventually leads to the presence of a high GSSG concentration in the surroundings. The nucleophilic cysteine moiety (Cys23) of the Grx1 enzyme of the biosensor interacts with the disulfide bond of GSSG forming an intermediate between Grx1 and GSSG (Meyer and Dick, 2010). This intermediate reacts with the cysteine residues of the roGFP2 making it to form S-glutathionylated roGFP2. It rearranges itself to finally form a disulfide bond between Cys147 and Cys204. Due to this disulfide bond formation in the roGFP2, conformational changes are observed. These changes subsequently lead to changes in the roGFP fluorescence excitation wavelengths at 405 and $488 \mathrm{~nm}$ respectively. This 3 step reaction (Fig. 3) is rapid and the sensor re-equilibrates as soon as the oxidation environment fades away.
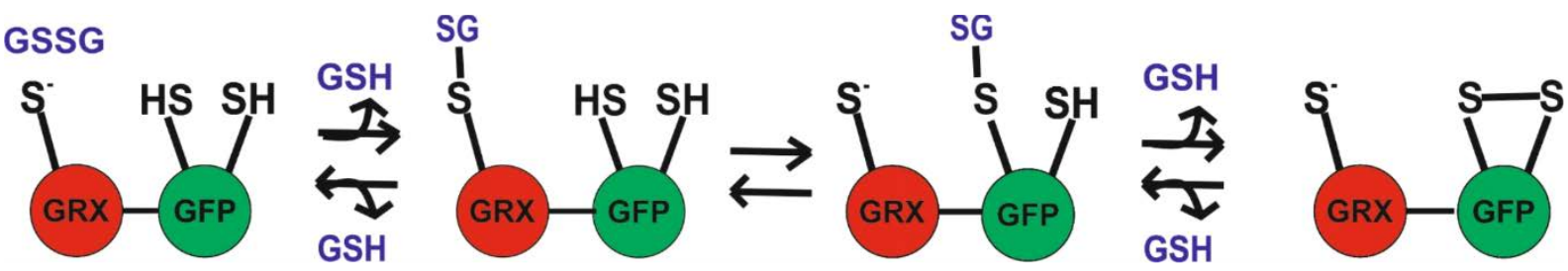

Figure 3: Working mechanism of the Grx1-roGFP2 biosensor. Shown is a graphical representation of the working mechanism of the Grx1-roGFP2 biosensor.

\section{OxyR based biosensor}

HyPer is a redox biosensor which can be used to detect specifically intracellular $\mathrm{H}_{2} \mathrm{O}_{2}$ (Belousov et al., 2006). The sensor includes the transcription factor OxyR (derived from E.coli) which is very sensitive to $\mathrm{H}_{2} \mathrm{O}_{2}$. The regulatory domain of the OxyR is linked with a circularly permutated yellow fluorescent protein (cpYFP) to form the HyPer biosensor (Fig. 4). In the presence of $\mathrm{H}_{2} \mathrm{O}_{2}$, there is a formation of a disulfide bond on the cysteine residues of OxyR Cys199 and Cys208. These redox changes occurring in the OxyR regulatory domain are transferred to the cpYFP. Due to the disulfide bond transfer to cpYFP, conformational changes are observed in the HyPer excitation wavelengths at 420 and $500 \mathrm{~nm}$ of the cpYFP. An 
increase in the $500 \mathrm{~nm}$ wavelengths and a decrease in $420 \mathrm{~nm}$ wavelength is observed upon oxidation. These changes can be recorded and can be used for analyzing the extent of $\mathrm{H}_{2} \mathrm{O}_{2}$ being present.
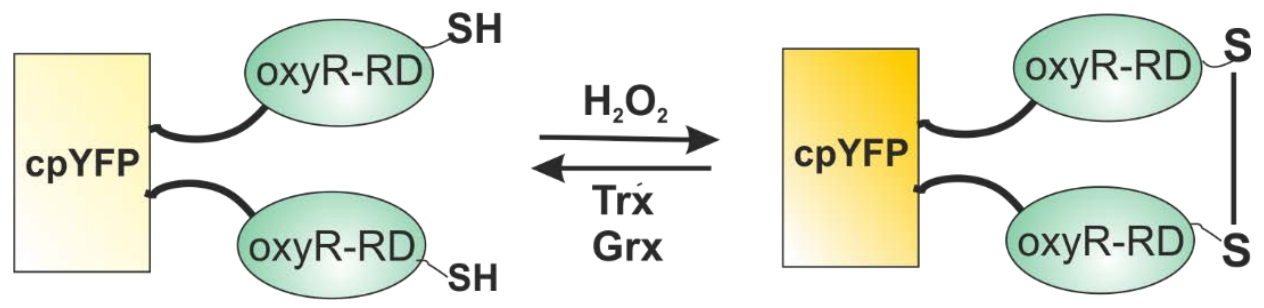

Figure 4: Schematic representation of the HyPer biosensor. The regulatory domain of OxyR (OxyR-RD) is linked to cpYFP. There is disulfide bond formation on OxyR-RD in presence of $\mathrm{H}_{2} \mathrm{O}_{2}$.

Compared to the dye based ROS detection, the above described genetically encoded biosensors allow real time visualization of the redox status of a cell along with a specific and quantitative ratiometric analysis of the redox potential of a cell. However, along with such advantages, the genetically encoded biosensors also possess some disadvantages. As described above, the synthetic probes are easy to use for measuring ROS in cultured cells with a simple plate reader assay. The genetically encoded biosensors are not that conveniently useful when it comes to using them with cultured cells. This is still quite possible with any kind of secondary cell line. The cells can be transfected with the desired redox biosensor and the transfected cell line can be further used for recording the redox status of the cell in any given experimental condition and treatment. However, this task becomes technically difficult when it has to be done on primary cells. Transduction of primary cells with the desired biosensor is a tedious process with respect to factors like time required for successful transduction can be longer which can affect the primary isolated cell's viability. Not only time, but also the transduction process itself can have harmful effects on the cell's overall health. Thus, in conclusion synthetic probes and redox biosensors indeed have their own associated advantages and disadvantages. However, the combination of in vivo study models with recent advances in the genetically encoded biosensors has made an attempt to direct the path of redox related studies closer to physiology.

\subsubsection{Glutathione redox potential measurements}

roGFP based sensors are in close equilibrium with the 2GSH/GSSG ratio in the given environment. This makes it possible to mathematically correlate electron flow between the roGFP and the GSH antioxidant system and calculate the $E_{\mathrm{GSH}}$ by using the Nernst equation as follows: 


$$
\begin{aligned}
E_{G S H} & =E_{G S H}^{\circ}-\frac{R T}{2 F} \ln \left(\frac{[\mathrm{GSH}] 2}{[G S S G]}\right) \\
& =E_{G S H}^{\circ}-\frac{R T}{2 F} \ln \left(\frac{[\text { roGFP2red }]}{[\text { roGFP2ox }]}\right) \\
& =E_{\text {roGFP2 }}
\end{aligned}
$$

Where $R$ is the gas constant $\left(8.315 \mathrm{~J} \mathrm{~K}^{-1} \mathrm{~mol}^{-1}\right), T$ the absolute temperature $(298.15 \mathrm{~K})$, and $F$

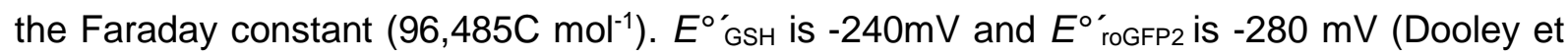
al., 2004).

Here, it is essential to understand the term Oxidation difference $(O x D)$. The total concentration of GSH $\left(G S H_{\text {total }}\right)$ is the overall GSH present $\left(G S H_{\text {total }}=[G S H]+[2 G S S G]\right)$. OxD $G S H$ refers to a fraction of $G S H_{\text {total }}$ that exists in the form of oxidized GSH [GSSG] (Meyer and Dick, 2010). For example, if the $O X D_{G S H}$ is 0.7 , it means the [GSSG] is $70 \%$ of all GSH are in the form of GSSG. In a biological system, it is useful to mathematically calculate the Nernst equilibrium relationship as a function of $O X D_{G S H}, G S H_{\text {total }}$ and $O x D_{\text {roGFP2: }}$

$$
\begin{aligned}
E_{G S H} & =E_{\text {roGFP2 }}^{\circ}-\frac{R T}{2 F} \ln \left(\frac{2 G S H_{\text {total }}\left(1-O x D_{G S H}\right)}{O x D_{G S H}}\right) \\
& =E_{\text {roGFP2 }}^{\circ}-\frac{R T}{2 F} \ln \left(\frac{1-O x D_{\text {roGFP2 }}}{O x D_{\text {roGFP2 }}}\right) \\
& =E_{\text {roGFP2 }}
\end{aligned}
$$

In order to calibrate the roGFP2 sensor, oxidizing and reducing agents like $\mathrm{H}_{2} \mathrm{O}_{2}$ and DTT are added externally to a particular cell. After addition of the respective reagents, a change in the excitation wavelengths of 405 and $488 \mathrm{~nm}$ is obtained. The ratio $\left(R_{\text {red }}\right.$ and $\left.R_{\text {oxd }}\right)$ between the excitation wavelengths is determined from the changes in the fluorescence intensities which define the total range of the sensor.

To understand the calculations for determining $O X D_{\text {roGFP2, }}$ it is important to know some quantities. $1405_{\text {red, }}$ i405ox, i488 red, and $1488_{o x}$ stands for the fluorescence intensities at the indicated wavelength and redox state for the roGFP2 molecules. $N_{\text {total }}$ is the total number of roGFP2 molecules, $N_{\text {red }}$ is the number of reduced roGFP2 molecules, and $N_{o x}$ is the number of oxidized roGFP2 molecules. OxD $\mathrm{D}_{\mathrm{roGFP} 2}$ can then be calculated from the following equation: 


$$
\begin{aligned}
& O x D_{\text {roGFP2 }}=\frac{N_{\text {ox }}}{N_{\text {total }}} \\
& O x D_{\text {roGFP } 2}=\frac{\text { I } 405 \text { x I 488red }- \text { I 405red X I } 488}{\text { I } 405 \text { x I 488red }- \text { I } 405 \text { x I 488ox }+ \text { I 405ox x I 488 - I 405red X I } 488}
\end{aligned}
$$

\subsection{Genetically encoded producer of reactive oxygen species}

Exogenous application of $\mathrm{H}_{2} \mathrm{O}_{2}$ is commonly used to study ROS signaling, which is not comparable to endogenous ROS effects. In addition, it might lack the possibility of mimicking the concentration of ROS produced in a physiological acceptable range. In order to go closer to physiology, there is recently a very basic but unique approach used to mimic the normal physiological concentration of ROS in the cells. In this approach, there is an involvement of an enzyme that can produce $\mathrm{H}_{2} \mathrm{O}_{2}$ endogenously when stimulated by its substrate (Pollegioni et al., 1993; Matlashov et al., 2014). The enzyme chosen is D-amino acid oxidase (DAAO), which can be activated by its substrate $\mathrm{D}$-alanine without disturbing major other pathways. This enzyme is fused to the $\mathrm{H}_{2} \mathrm{O}_{2}$ biosensor HyPer which can in turn detect the extent of $\mathrm{H}_{2} \mathrm{O}_{2}$ produced by the enzyme DAAO. DAAO is derived from yeast Rhodotorula gracilis and can catalyze the reaction which results in the production of $\mathrm{H}_{2} \mathrm{O}_{2}$ (Fig. 5).

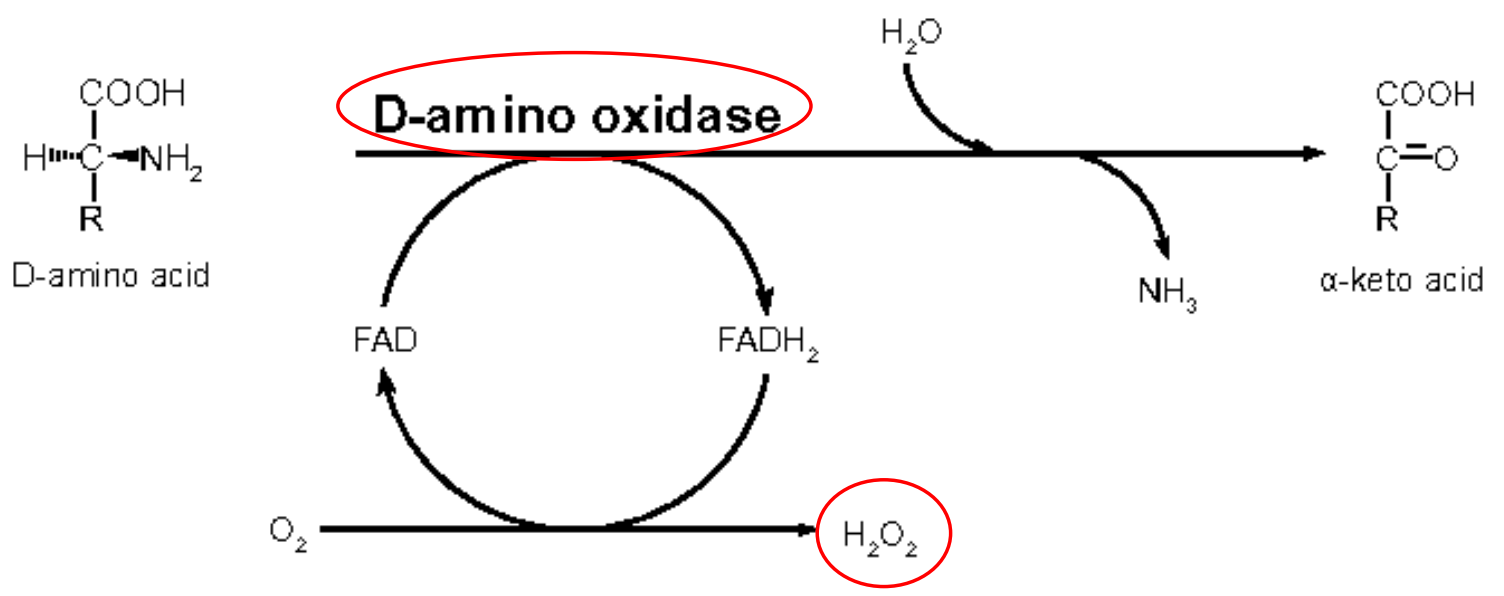

Figure 5: Chemical reaction catalyzed by D-amino acid oxidase (DAAO). DAAO converts its substrate D-amino acid to $\alpha$-keto acid at the expense of an electron from FAD. FAD is redoxidized back to $\mathrm{FADH}_{2}$ by molecular oxygen along with the generation of $\mathrm{H}_{2} \mathrm{O}_{2}$. (Adapted from (Chen et al., 2013))

In mammalian cells, the enzyme DAAO is hardly present. This makes it possible to use DAAO as a genetically engineered ROS producer in mammalian cells. $\mathrm{H}_{2} \mathrm{O}_{2}$ gives an effect locally. The fusion protein DAAO-Hyper can also be localized into different compartments of the cell using relevant targeting signals (Bogdanova et al., 2017). For instance, the fusion protein can be targeted to the nucleus of a cell by using a nuclear localization signal (NLS). Short signal 
peptides added on to the C-terminus of the protein can guide the nuclear transport systems in or out of the nucleus. With this concept, the fusion protein can be targeted to the nucleus by NLS or outside the nucleus by the nuclear export signal (NES). Depending on the localization of the sensor, the concentration of $\mathrm{H}_{2} \mathrm{O}_{2}$ produced can vary from nanomolar range to submicromolar range. For example, when D-alanine as low as $0.25 \mathrm{mM}$ is added to HeLaKyoto cells expressing DAAO in the nucleus (DAAO-NLS), lower amounts to $\mathrm{H}_{2} \mathrm{O}_{2}$ levels are recorded by the HyPerRed-NLS. However, such low amounts cannot exit the nucleus and thus cannot be detected by HyPer2-NES present in the same set of cells. On the other hand, when D-alanine of $0.6 \mathrm{mM}$ is added to HeLa-Kyoto cells expressing DAAO outside the nucleus (DAAO-NES), it produces $\mathrm{H}_{2} \mathrm{O}_{2}$ sufficiently high to get detected by HyPerRed-NLS as well as HyPer2-NES. At the same time, the $\mathrm{H}_{2} \mathrm{O}_{2}$ produced by DAAO is lower than $50-100 \mathrm{nM}$ and this is close to a physiologically acceptable range (Matlashov et al., 2014).

\subsection{In vivo models for Grx1-roGFP2 and HyPer based redox biosensors}

In recent years, several in vivo roGFP based models were generated (Swain et al., 2018). Transgenic drosophila flies were genetically modified by inserting a Grx1-roGFP2 biosensor which either localized in the cytoplasm or got targeted to the mitochondria (Albrecht et al., 2011). The expression of the sensor was controlled under the Tubulin promoter. This allowed a real time visualization of compartment specific $E_{\mathrm{GSH}}$. A transgenic $C$. elegans was developed having the redox biosensor Grx1-roGFP2 which was controlled by rx1the large ribosomal subunit L17 promoter (Back et al., 2012). This was the first in vivo transgenic redox biosensor nematode to be developed.

With regards to redox sensor based transgenic mouse models, there were several transgenic mouse models developed ranging from neuronal specific, erythrocyte specific, cardiomyocyte specific roGFP sensors. A Grx1-roGFP2 biosensor targeted to the mitochondrial matrix which expresses under the thymocyte differentiation antigen/CD90 (Thy-1.2) promoter was developed (Breckwoldt et al., 2014). This mouse model proved to be of great use for studying the redox changes in mitochondrial matrix linked to neuronal diseases.

Most notably, a cardiomyocyte-specific Grx1-roGFP2 mouse model was developed wherein the sensor is in the cytoplasm and also targeted to the mitochondrial matrix (by an ATP synthase 9 mitochondrial signal) of the cardiomyocytes (Swain et al., 2016). The sensor is expressed under the control of $\alpha-\mathrm{MHC}$ promoter making it cardiomyocyte specific. This sensor was utilized to analyze the physiological $E_{\mathrm{GSH}}$ of the two compartments: cytoplasm and mitochondrial matrix in the cardiomyocytes isolated by Langendorff's perfusion system. 
Interestingly, it was observed that the mitochondrial matrix is more reduced ( $E_{\mathrm{GSH}}=-278.9 \mathrm{mV}$ ) compared to the cytoplasm ( $\left.E_{\mathrm{GSH}}=-254.8 \mathrm{mV}\right)$. This showed that there is an existence of redox compartmentalization in cardiomyocytes.

Very recently, an in vivo rat model was developed wherein the HyPer based redox biosensor was transduced into the rats with the help of an adeno-associated virus serotype 9 vector (Steinhorn et al., 2018). The sensor was driven under the cardiac troponinT (cTnT) promoter making it specific to cardiomyocytes. It comprises a fusion protein of the enzyme DAAO along with the HyPer biosensor. When DAAO is activated by external addition of D-alanine as a substrate to the enzyme in the cardiomyocytes, there is an internal production of $\mathrm{H}_{2} \mathrm{O}_{2}$ which can be in turn detected by the HyPer probe (Fig.6). This in vivo model proved to be of very high importance in studying the effect of production of ROS in the heart. It could show a direction towards how ROS can induce a dilated cardiomyopathy with significant systolic dysfunction.

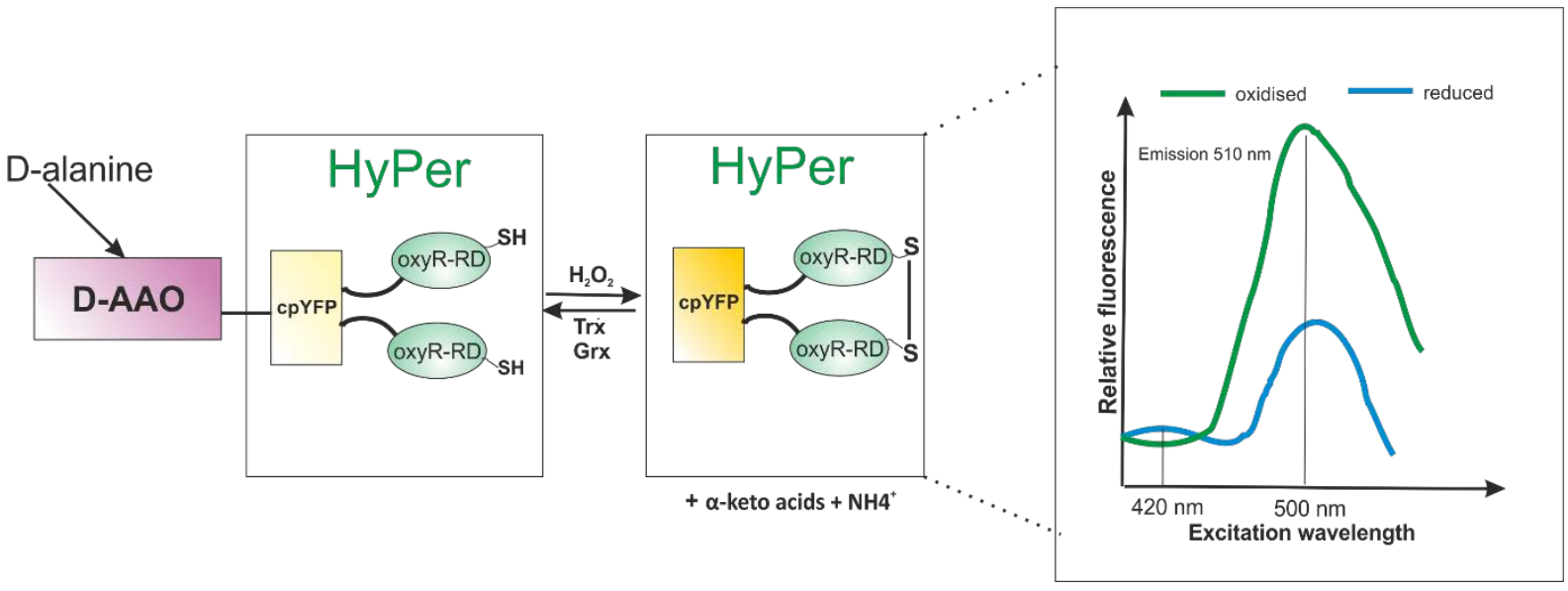

Figure 6: Schematic representation of the DAAO-HyPer biosensor.

\subsection{Mitochondrial redox players and their significance}

There is a very strong and influential network within several players having an equally important role and leading to a great impact on maintaining the physiological redox status in the mitochondrial matrix (Fig. 7). These players can be listed as follows: GSH, NADPH, $\mathrm{NADH} / \mathrm{NAD}^{+}, \mathrm{ETC}$ substrates, ROS, molecular $\mathrm{O}_{2}$. 


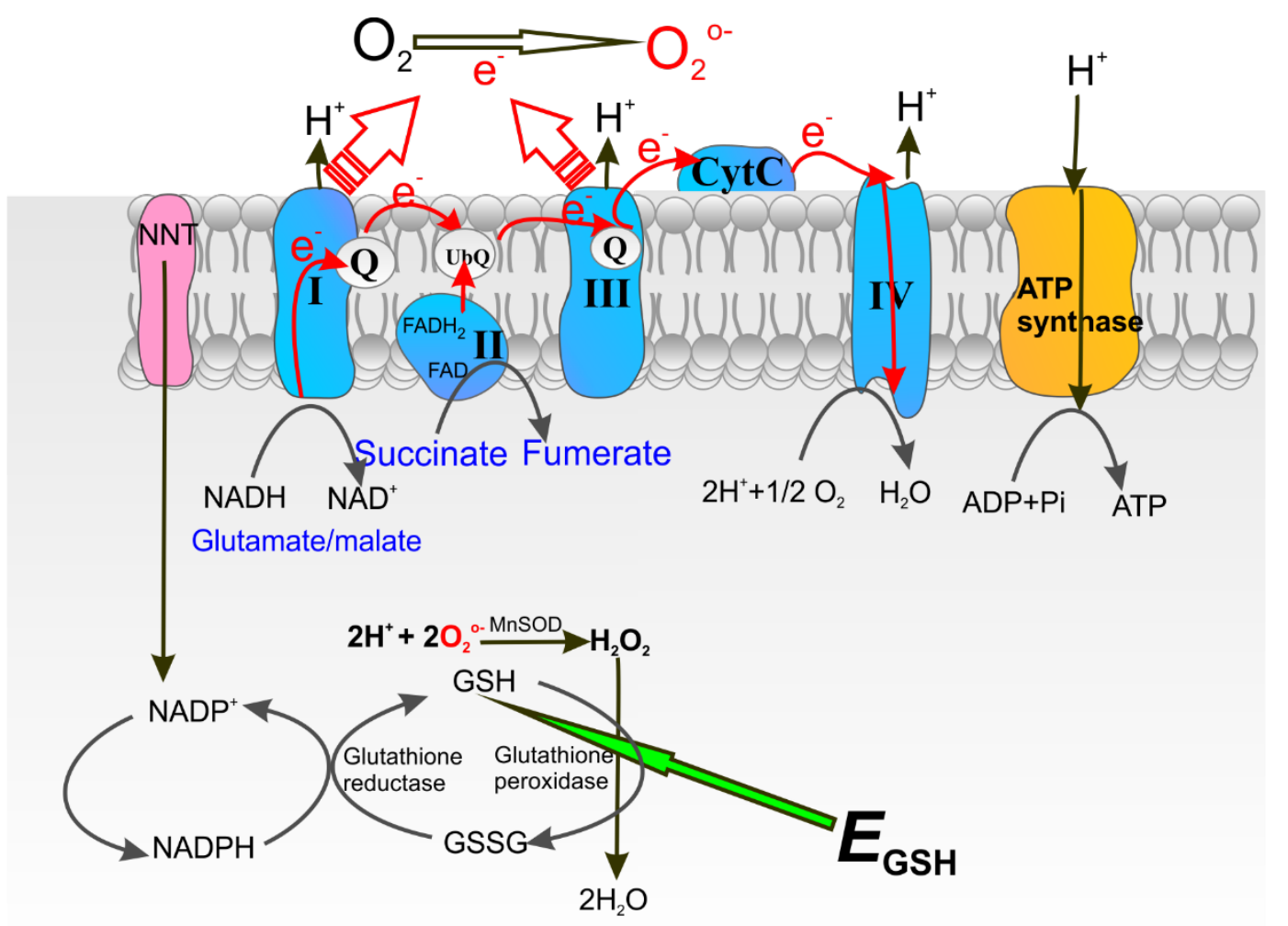

Figure 7: Schematic representation of a mitochondrial electron transport chain. Glutathione (GSH), NADPH, NADH/NAD ${ }^{+}$, ETC substrates, ROS, molecular oxygen are the players taking part in the entire mitochondrial ETC which finally have a huge impact of the $E_{G S H}$ of the mitochondrial matrix.

As introduced above, mitochondria are indeed one of the major ROS generators in a cell. Grx is one of the important antioxidant system mediating the redox process in the mitochondria. $\mathrm{NADPH}$ is the central player in maintaining the redox buffer working in co-operation with the GSH antioxidant system. The major source of NADPH is nicotinamide nucleotide transhydrogenase (NNT), which uses NADH for the generation of NADPH from NADP ${ }^{+}$. There are also some other sources of $\mathrm{NADPH}$ like malic enzymes and $\mathrm{NADP}^{+}$-isocitrate dehydrogenase (Rydström, 2006). Thus, the generation of NADPH is indirectly dependent on the generation of NADH in the ETC as well. Substrates like succinate, glutamate, and malate stimulate the generation of $\mathrm{NADH}$ and in turn NADPH. NADH/NAD ${ }^{+}$ratio influences the ROS produced especially in complex I and III of the ETC. Complex II, commonly known as succinate dehydrogenase is an enzyme which is a part of both citric acid cycle and oxidative phosphorylation. When isolated mitochondria are stimulated with succinate as a substrate, succinate gets oxidized to fumarate and eventually leads to the production of a high amount of $\mathrm{NADH}$ and $\mathrm{NADPH}$ as reducing equivalents. This results in high $\mathrm{GSH}$ content in the mitochondrial matrix restoring the mitochondria to maintain its reduced redox status. Thus, one of the ways to restore the redox status due to oxidative stress created by the ROS produced in the complex I and III can be by stimulating the mitochondria with substrates like succinate 
(Garcia et al., 2010). ROS produced in the form of ${ }^{\circ} \mathrm{O}_{2}{ }^{-}$is also dependent on the availability of the molecular $\mathrm{O}_{2}$. Thus, it can be understood that all these players are internally strongly linked to each other and disturbance of even a single player can lead to alteration in the redox status of the mitochondria.

\subsection{Molecular oxygen and mitochondria: an ancient relation}

Over three billions years ago a primitive cellular life originated on earth. Gradually, prokaryotic cellular life evolved in the Earth's ocean in the form of cyanobacteria which utilized sunlight for metabolism and photosynthesis (Semenza, 2007). This gave rise to molecular $\mathrm{O}_{2}$ which not only proved to be highly reactive but also could produce a huge number of toxic by-products. $\mathrm{O}_{2}$ being a reactive species got incorporated into the inorganic minerals present on the earth's crust by oxidation (Dole, 2004). The oxidation of these inorganic minerals reached a point where no more oxidation could occur. Thereafter, molecular $\mathrm{O}_{2}$ started accumulating in the atmosphere. The $\mathrm{O}_{2}$ which initially proved to be toxic to lower organisms due to oxidation, later proved to be the most essential moiety of life in complex organisms like eukaryotes. As a result of the eventual endosymbiosis, mitochondria are now one of the organelles present in a eukaryotic cell. Mitochondria being responsible for the production of ATP by using the molecular oxygen proved to be essential for the development of multicellular organisms and aerobic metabolism (Morrison, 2009).

Molecular oxygen is an essential player in cell metabolism. Mitochondria consume $\mathrm{O}_{2}$ to produce ATP by the process called oxidative phosphorylation in aerobic cellular respiration. A human body takes up $\mathrm{O}_{2}$ through the inhaled air in the lungs. Further, it makes an entry into the heart with the blood stream bound to hemoglobin and then finally reaches all other tissues. Till the time $\mathrm{O}_{2}$ diffuses into different tissues the partial oxygen pressure $\left(\mathrm{pO}_{2}\right)$ eventually decreases as compared to the atmospheric $\mathrm{pO}_{2}$ at sea level. The overall $\mathrm{pO}_{2}$ within various organs is in the range of $25-65 \mathrm{~mm} \mathrm{Hg}$ (Vaupel et al., 1989). Hypoxia is the phenomenon that occurs when the oxygen level in the organs drops down below the respective $\mathrm{pO}_{2}$. Alternatively, it arises when the $\mathrm{O}_{2}$ consumption rate of a cell is higher than the available $\mathrm{O}_{2}$.

From the organ level to the cellular level and subsequently to the organelle level, the $\mathrm{pO}_{2}$ within the organelles becomes absolutely low. Therefore, mitochondria are exposed to lower oxygen concentrations with a steep drop of the $\mathrm{pO}_{2}$ around each mitochondria in a normal physiological environment (Sinaasappel et al., 2006). Mitochondria function mostly as oxygen sinks (Wenger, 2006). The $\mathrm{pO}_{2}$ experienced by the mitochondria is $<1-3 \mathrm{~mm} \mathrm{Hg}$ in cells under atmospheric normoxic conditions. 
Mitochondria are naturally under lower oxygen conditions and they function efficiently in physiological in situ conditions with this low $\mathrm{pO}_{2}$. As seen in earlier sections, molecular $\mathrm{O}_{2}$ and mitochondria have a very close relationship in regards to ROS production. Still, most of the studies on mitochondrial functions are done in isolated mitochondria, where the isolated mitochondria are exposed to higher $\mathrm{pO}_{2}$ than the naturally occurring $\mathrm{pO}_{2}$ (Kurtcuoglu et al., 2015). Therein, altering the physiological $\mathrm{pO}_{2}$ experienced for the mitochondria is being grossly neglected.

\subsection{Mitochondrial calcium uniporter (MCU)}

Calcium $\left(\mathrm{Ca}^{2+}\right)$ is one of the significant signaling molecules occurring in the mitochondrial intermembrane space (IMS). A concrete relation is observed between the $\mathrm{Ca}^{2+}$ homeostasis and the mitochondrial redox status by relaying $\mathrm{H}_{2} \mathrm{O}_{2}$ signals and $\mathrm{Ca}^{2+}$ signaling in the mitochondria (Petrungaro et al., 2015). Here comes the role of an IMS $\mathrm{Ca}^{2+}$ sensitive ion channel called mitochondrial calcium uniporter (MCU) into play (Kirichok et al., 2004). MCU takes up the $\mathrm{Ca}^{2+}$ from the outer mitochondrial membrane. In consequence of the increased $\mathrm{Ca}^{2+}$ levels, $\mathrm{Ca}^{2+}$-sensitive dehydrogenases are activated to initiate ATP synthesis (Glancy and Balaban, 2012).

The MCU complex consists of a protein structure containing 3 core components namely MCU, MCUb and EMRE (Girgis et al., 2010). MCU consists of two coiled-coil domains and two transmembrane domains separated by a short acidic rich loop. MCUb is a similar protein and shares 50\% homology to MCU. Essential regulatory subunit of mitochondrial calcium uniporter (EMRE) is the third member of the MCU complex. The $\mathrm{Ca}^{2+}$ channel is highly regulated by the MICU family. As shown in Fig. 8, the members of this protein family have a gatekeeper role, i.e. keeping the channel closed at resting conditions and upon activation at high $\mathrm{Ca}^{2+}$ concentrations allowing the $\mathrm{Ca}^{2+}$ entry through the MCU complex. MICU1 was the first family member to be discovered. It acts as a gatekeeper for the $\mathrm{Ca}^{2+}$ entry inside the channel. MICU1 has two relatives namely MICU2 and MICU3. MICU2 also faces the IMS forming a heterodimer with MICU1. MICU2 is known to have inhibitory effects whereas MICU1 activates the channels which guarantees rapid $\mathrm{Ca}^{2+}$ accumulation at the same time preventing $\mathrm{Ca}^{2+}$ overload (Mantoan et al., 2014). MICU3 has a minor role and is significantly only expressed in CNS. 

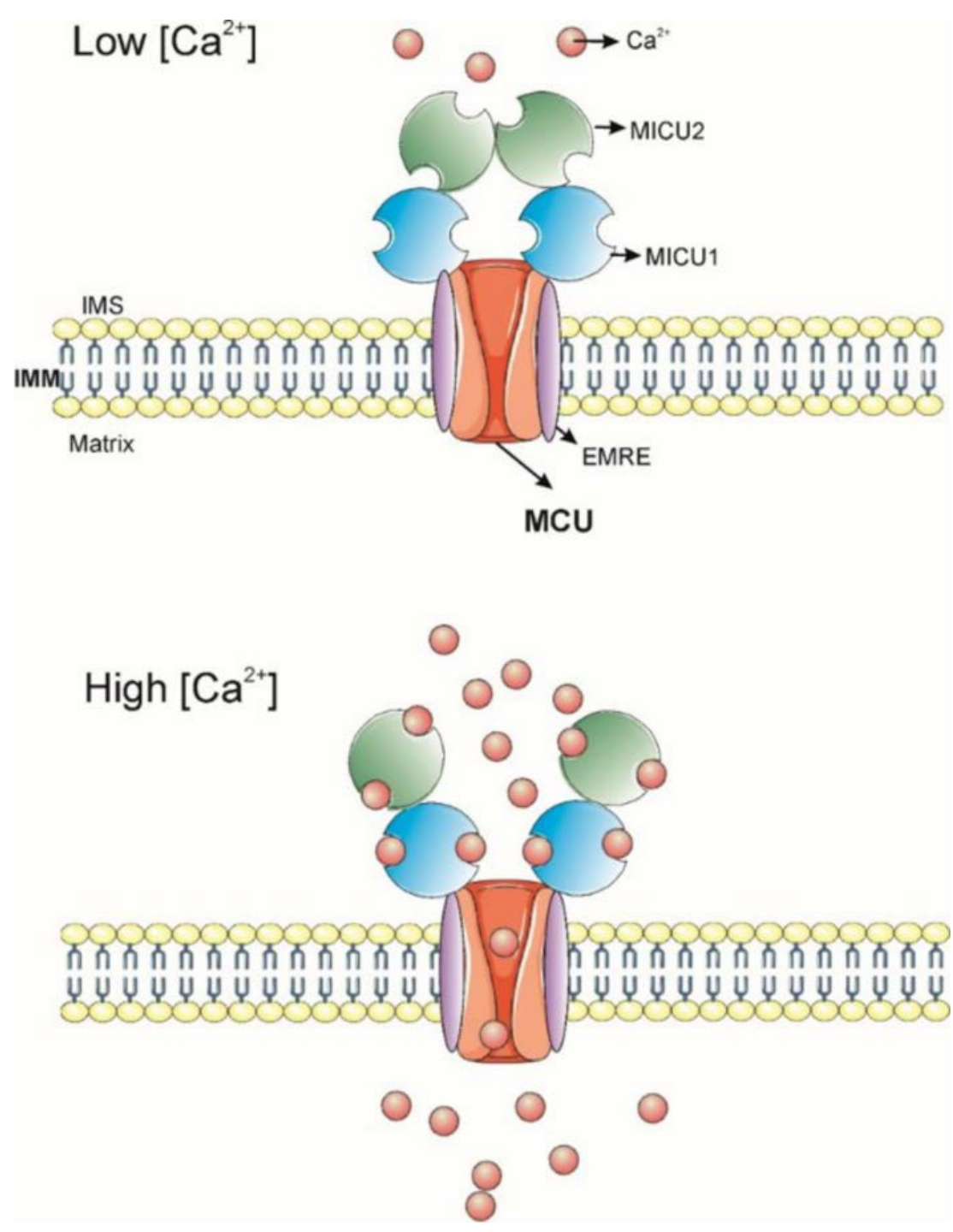

Figure 8: Schematic representation of the working mechanism of MCU complex.

The oxidoreductase Mia40 is a mediator in the formation of a disulfide bond between MICU1MICU2 and further fine tunes the $\mathrm{Ca}^{2+}$ uptake by the MCU (Petrungaro et al., 2015). The interaction partner for Mia40 is MICU1 where it introduces a disulfide bond which links MICU1 and MICU2. This dimer formation makes the binding of MICU1-MICU2 to MCU possible. At resting $\mathrm{Ca}^{2+}$ concentrations, the dimer associates with $\mathrm{MCU}$ whereas at high $\mathrm{Ca}^{2+}$ concentrations the dimer dissociates from $\mathrm{MCU}$ allowing $\mathrm{Ca}^{2+}$ entry.

There seems to be an indirect correlation between the mitochondrial redox status and $\mathrm{Ca}^{2+}$ homeostasis via Mia40 as an interaction partner to MICU1. Eventually, this will have a huge impact on the regulation of ATP synthesis in mitochondria by MCU. 


\subsection{Redox signaling in cardiomyocytes}

Redox signaling plays a major role in physiological cardiac function, however also in the development of heart failure. The cellular sources of ROS in cardiomyocytes are similar to other cell types, i.e. mitochondrial and NOX-dependent ROS production is observed. Among the different NOX isoforms, Nox2 and Nox4 are mainly expressed in cardiomyocytes (Looi et al., 2008). Although similar in structure, Nox2 and Nox4 mediate distinct redox signaling pathways in cardiomyocytes, which are in part even contrasting in the context of heart failure. This may relate to major differences between the two isoforms regarding cellular localization, activation and target molecules. Activated Nox2 is predominantly expressed at the plasma membrane (Heymes et al., 2003), whereas Nox4 is found intracellularly in the sarcoplasmic reticulum although the precise location still remains controversial. Nox2 requires activation by agonists to form the fully active enzyme complex (El Benna et al., 1996), whereas Nox4 is constitutively active producing low levels of ROS under basal conditions (Wang et al., 2010). Nox2 produces ${ }^{\circ} \mathrm{O}_{2}{ }^{-}$; Nox4 has the ability to generate predominantly $\mathrm{H}_{2} \mathrm{O}_{2}$ instead (Wang et al., 2010). This has major implications for the half-life of the Nox2/4-induced effects as well as for the interaction with nitric oxide (NO) signaling. Whereas Nox2 produced ${ }^{\circ} \mathrm{O}_{2}{ }^{-}$can limit $\mathrm{NO}$ signaling by generating peroxnitrite, the Nox4 produced $\mathrm{H}_{2} \mathrm{O}_{2}$ might even enhance $\mathrm{NO}$ signaling by stimulating the activity of nitric oxide synthetase. In the context of cardiac diseases like chronic pressure overload, Nox4 promotes adaptive cardiac remodelling through effects that include a preservation of myocardial capillary density (Zhang et al., 2010; Zhang et al., 2018) and reprogramming of cardiac substrate metabolism (Nabeebaccus et al, 2017). Nox2, on the other hand seems to augment cardiac hypertrophy and adverse cardiac remodeling.

There are several redox-sensitive molecular targets described in cardiomyocytes. These include proteins of the excitation-contraction coupling as well as well as the G-protein coupled receptor signaling machineries. Excitation-contraction coupling (ECC) involves a fine-tuned interaction of several channels that regulate calcium $\left(\mathrm{Ca}^{2+}\right)$ homeostasis and thus cardiomyocyte contraction (Köhler et al., 2014). Among those the ryanodine receptor and phospholamban are known to be targeted by ROS, which alters their functionality. ECC is under the control of $\beta$-adrenergic signaling. Protein kinase $A$ (PKA), Protein kinase $G(P K G)$ and the $\mathrm{Ca}^{2+} /$ calmodulin-dependent protein kinase II (CaMKII) are stimulated by $\beta$-receptor activation. All three kinases have been described to be redox-sensitive, which in addition affects $\mathrm{Ca}^{2+}$ homeostasis and cardiomyocyte contraction.

Aside from ECC, redox signaling seems to affect directly gene expression in the nucleus (Backs et al., 2006). Gene expression is controlled by the acetylation and deacetylation of 
histones. Acetylation removes the positive charge on histones, thereby decreasing their interaction with the negatively charged phosphate groups of DNA. The resulting condensed chromatin is transformed into a more relaxed structure that allows greater levels of gene transcription. In line, transcriptional activity can be fostered either by increased acetylation activity or by blocking histone deacetylases (HDAC). HDAC are expressed literally in all human cells. In cardiomyocytes HDAC4 is the most prevalent isoform. Its deacetylation activity is regulated by nucleocytoplasmic shuttling that is stimulated by phosphorylation. The phosphorylation of HDAC4 leads to its nuclear export, which is mediated via the interaction with the signaling molecule 14-3-3 and the exportin CRM1. HDAC4 has been directly associated with the gene expression of nuclear factor of activated T cell (NFAT) and the myocyte enhancer factor 2 (MEF2). Both are major regulators of cardiac hypertrophy. Upon redox-modification of critical cysteine residues in HDAC4 its localization is affected, which results in an important link between redox signaling and cardiac hypertrophy development.

Although redox signaling is important for cardiac function, the lack of suitable chemical ROS sensitive dyes or genetically encoded biosensor models, did not allow describing specific redox pathways in cardiomyocytes in more detail. Especially compartment-specific alterations of ROS or determining the $E_{\mathrm{GSH}}$ in cardiomyocytes was technically not possible. Dr. Lija Swain (Institute of Cardiovascular Physiology, Göttingen) has established cardiomyocyte-specific transgenic mice expressing Grx1-roGFP2 either in the mitochondrial matrix or the cytoplasm in the past. These mice proved to be useful to analyze the compartment-specific $E_{\mathrm{GSH}}$ in isolated whole hearts as well as isolated adult cardiomyocytes. Most importantly this animal model allowed demonstrating that the cytosol and the mitochondrial matrix indeed have distinct $E_{\mathrm{GSH}}$. Having a $E_{\mathrm{GSH}}$ biosensor mouse model established was the basis to apply it to open questions regarding patho-mechanisms in the heart in this thesis.

\subsection{Aims of the thesis}

Redox signaling in cardiomyocytes is important for their physiological function, however is also associated with the development of heart failure. GSH is an important buffer to counteract increased production of ROS. Therefore the $E_{\mathrm{GSH}}$ is a good marker for alterations in redox signaling. Since recently a cardiomyocyte-specific transgenic mouse model is available using the genetically encoded Grx1-roGFP2 biosensor for analyzing the $E_{\mathrm{GSH}}$. This essential in vivo tool was applied in the thesis to analyze the $E_{G S H}$ during cardiac ageing. In line the first objective of the thesis was: 
i. Characterization of the in vivo transgenic mouse model aMHC-Grx1-roGFP2, in which the biosensor is either localized in the cytoplasm or targeted to the mitochondrial matrix. To gain insight into cardiac ageing processes young versus ageing mice were included in the analysis.

An initial hint was observed over the course of investigating the first objective, that the normal physiological environment of the mitochondria does play an important role in maintaining the $E_{\mathrm{GSH}}$ of that organelle. Corresponding to this interpretation, the second objective of the thesis was:

ii. To study the role of molecular $\mathrm{O}_{2}$ as one of the major key players in helping to preserve the mitochondrial $E_{\mathrm{GSH}}$ as well as mitochondrial functionality ex vivo

Endogenous redox manipulation was done in the Grx1-roGFP2 mouse model by adding $\mathrm{H}_{2} \mathrm{O}_{2}$ exogenously. This does not mimic ROS production from endogenous sources. Therefore I aimed to upgrade the in vivo redox biosensor tool box by applying an endogenous ROS generator in combination with a biosensor for transgenesis in mice. Accordingly the third aim of the thesis was:

iii. To generate and characterize transgenic cardiomyocyte-specific DAAO-HyPer mice. 


\section{Materials and methods}

\subsection{Materials}

\subsubsection{Chemicals and reagents}

Following were the chemicals and reagents used for this work:

Table 3: Chemical and reagents used.

\begin{tabular}{|c|c|c|}
\hline Chemicals & Manufacturer & Article number \\
\hline $\begin{array}{l}\text { e-Amino n-caproic } \\
\text { acid }\end{array}$ & Sigma & A2504 \\
\hline $2.5 \%$ Trypsin & Gibco & 15090 \\
\hline Acetic acid & Carl Roth $\mathrm{GmbH}$ & 3738.5 \\
\hline Acrylamide & Carl Roth $\mathrm{GmbH}$ & 7871.2 \\
\hline ADP & Sigma & A2754 \\
\hline Agarose Broad Range & Carl Roth $\mathrm{GmbH}$ & T846.3 \\
\hline Antimycin A & Sigma & A8674 \\
\hline APS & Carl Roth $\mathrm{GmbH}$ & 9592.1 \\
\hline BDM & Sigma & B0753 \\
\hline Bis-Tris/HCl, pH 7.0 & Carl Roth $\mathrm{GmbH}$ & 9140.3 \\
\hline Bis-acrylamide & Serva & 29195.03 \\
\hline Boric acid & Carl Roth GmbH & 6943.1 \\
\hline BSA & Sigma & A8806 \\
\hline $\mathrm{CaCl}_{2}$ & Carl Roth $\mathrm{GmbH}$ & CN93.1 \\
\hline Coomassie G & Carl Roth $\mathrm{GmbH}$ & 9598.2 \\
\hline Cytochrome c & Calbiochem & 250600 \\
\hline D-Alanine & Sigma & A7377 \\
\hline Decylubichinone & Sigma & D7911 \\
\hline Diamide & Sigma & D3648 \\
\hline Digitonin & Merck & 300410 \\
\hline $\begin{array}{l}\text { DL-Malic acid } \\
\text { disodium salt }\end{array}$ & Sigma & M6773 \\
\hline DTT & Carl Roth $\mathrm{GmbH}$ & 6908.1 \\
\hline ECL & Thermo Scientific & 32106 \\
\hline EDTA & Carl Roth $\mathrm{GmbH}$ & 8043.2 \\
\hline Ethanol & Carl Roth GmbH & 3738.5 \\
\hline
\end{tabular}




\begin{tabular}{|c|c|c|}
\hline $\begin{array}{l}\text { Gene Ruler DNA } \\
\text { Ladder } 1 \mathrm{~kb}\end{array}$ & Thermo Scientific & SM0311 \\
\hline Glucose & Carl Roth $\mathrm{GmbH}$ & X997.2 \\
\hline Glycerol & Sigma & G7757 \\
\hline $\mathrm{H}_{2} \mathrm{O}_{2}$ & Carl Roth $\mathrm{GmbH}$ & 8070.2 \\
\hline $\mathrm{HCl}$ & Carl Roth $\mathrm{GmbH}$ & K025.1 \\
\hline HEPES & Carl Roth GmbH & 9105.2 \\
\hline $\mathrm{KCL}$ & Carl Roth GmbH & 6781.1 \\
\hline $\mathrm{KH}_{2} \mathrm{PO}_{4}$ & Carl Roth $\mathrm{GmbH}$ & 6875.1 \\
\hline $\mathrm{KHCO}_{3}$ & Sigma & 237205 \\
\hline L-Alanine & Sigma & A7627 \\
\hline Laminin & Sigma & L2020 \\
\hline $\begin{array}{l}\text { L-Glutamic acid } \\
\text { monosodium salt } \\
\text { hydrate }\end{array}$ & Sigma & G1626 \\
\hline Liberase solution & Roche & 5401054001 \\
\hline Mannitol & Carl Roth $\mathrm{GmbH}$ & 4175.1 \\
\hline Methanol & Carl Roth $\mathrm{GmbH}$ & 4627 \\
\hline $\mathrm{MgSO}_{4}, 7 \mathrm{H}_{2} \mathrm{O}$ & Carl Roth GmbH & P027.2 \\
\hline MOPS & Carl Roth GmbH & 6979.2 \\
\hline $\mathrm{Na}_{2} \mathrm{HPO}_{4}, 2 \mathrm{H}_{2} \mathrm{O}$ & Carl Roth GmbH & 49841 \\
\hline $\mathrm{NaCl}$ & Carl Roth GmbH & 9265.2 \\
\hline $\mathrm{NaHCO}_{3}$ & Carl Roth GmbH & HN01.1 \\
\hline $\mathrm{NaOH}$ & Carl Roth $\mathrm{GmbH}$ & 6771.1 \\
\hline $\begin{array}{l}\text { n-Dodecyl-beta- } \\
\text { Maltoside }\end{array}$ & Calbiochem & 324355 \\
\hline NEM & Sigma & E3876 \\
\hline Orange $\mathrm{G}$ dye & Carl Roth GmbH & O318 \\
\hline Paraformaldehyde & Carl Roth $\mathrm{GmbH}$ & O335.1 \\
\hline $\begin{array}{l}\text { Phenol red sodium } \\
\text { salt }\end{array}$ & Sigma & P5530 \\
\hline PMSF & Carl Roth $\mathrm{GmbH}$ & 6367 \\
\hline Potassium cyanide & Fluka & 60178 \\
\hline Potassium phosphate & Sigma & P5629 \\
\hline Roti Safe Gel Stain & Carl Roth $\mathrm{GmbH}$ & $3865 . .1$ \\
\hline
\end{tabular}




\begin{tabular}{|l|l|l|}
\hline $\begin{array}{l}\text { Sodium succinate } \\
\text { dibasic hexahydrate }\end{array}$ & Sigma & S2378 \\
\hline Succrose & Carl Roth $\mathrm{GmbH}$ & 4621.1 \\
\hline Taurine & Sigma & T8691 \\
\hline TEMED & Carl Roth $\mathrm{GmbH}$ & 2367.2 \\
\hline $\begin{array}{l}\text { To-Pro-3 lodide } \\
\text { nuclear stains }\end{array}$ & Molecular probes & T3605 \\
\hline Tricine, pH 7.0 & Carl Roth $\mathrm{GmbH}$ & 6977.3 \\
\hline Tris & Carl Roth $\mathrm{GmbH}$ & 5429.3 \\
\hline Tris/HCl, pH 7.4 & Carl Roth $\mathrm{GmbH}$ & 9090 \\
\hline Tris/HCl, pH 5.0 & Carl Roth $\mathrm{GmbH}$ & 9090.1 \\
\hline Tween 20 & Carl Roth $\mathrm{GmbH}$ & 9127 \\
\hline
\end{tabular}

\subsubsection{Assay kits}

Table 4: PCR master mix for genotyping.

\begin{tabular}{|l|l|l|}
\hline Chemicals & \multicolumn{2}{|l|}{ Manufacturer } \\
\hline PCR master mix (2x) & $\begin{array}{l}\text { roGFP mouse } \\
\text { line PCR }\end{array}$ & $\begin{array}{l}\text { DAAO-HyPer } \\
\text { mouse line PCR }\end{array}$ \\
\cline { 2 - 3 } & $\begin{array}{l}\text { Bioline (2x MyFi } \\
\text { mix) }\end{array}$ & $\begin{array}{l}\text { Thermo scientific } \\
\text { (dream Taq } \\
\text { master mix 2x) }\end{array}$ \\
\hline
\end{tabular}

Table 5: ATP measurement assay kit.

\begin{tabular}{|l|l|l|}
\hline Chemicals & Manufacturer & Article number \\
\hline $\begin{array}{l}\text { Celltiter-Glo } \\
\text { luminescent cell } \\
\text { viability assay kit }\end{array}$ & Promega & G7571 \\
\hline
\end{tabular}

Table 6: Protein estimation assay kit.

\begin{tabular}{|l|l|l|}
\hline Chemicals & Manufacturer & Article number \\
\hline $\begin{array}{l}\text { Bradford Protein } \\
\text { Reagent }\end{array}$ & Bio-Rad & 5000006 \\
\hline
\end{tabular}


Table 7: MitoSox Red dye.

\begin{tabular}{|l|l|l|}
\hline Chemicals & Manufacturer & Article number \\
\hline MitoSOX Red & Invitrogen & M36008 \\
Superoxide Indicator & & \\
\hline
\end{tabular}

Table 8: Amplex UltraRed dye.

\begin{tabular}{|l|l|l|}
\hline Chemicals & Manufacturer & Article number \\
\hline $\begin{array}{l}\text { Amplex UltraRed } \\
\text { reagent }\end{array}$ & Invitrogen & A36006 \\
\hline $\begin{array}{l}\text { Superoxide dismutase } \\
\text { (SOD) }\end{array}$ & Sigma & S9637-30KU \\
\hline $\begin{array}{l}\text { Horse radish } \\
\text { peroxidase (HRP) }\end{array}$ & Sigma & P6782-5MG \\
\hline
\end{tabular}

\subsubsection{Primer list}

Table 9: Primers used for genotyping.

\begin{tabular}{|c|c|c|c|}
\hline Gene of interest & \multicolumn{2}{|c|}{ Sequence } & $\begin{array}{l}\text { Annealing } \\
\text { temperature }\left({ }^{\circ} \mathrm{C}\right)\end{array}$ \\
\hline \multirow{2}{*}{ roGFP } & fw & 5'-СССТСТСTTTCTCTGCCCAG-3' & \multirow{2}{*}{58} \\
\hline & rev & 5'-ATAAAGACTCGAGGCACCGT-3' & \\
\hline \multirow{2}{*}{ PHD2 } & fw & 5'-CTCACTGACCTACGCCGTGT-3' & \multirow{2}{*}{58} \\
\hline & rev & 5'-CGCATCTTCCATCTCСАTTT-3' & \\
\hline \multirow{2}{*}{ DAAO-NES } & fw & 5'-CCTGAGACCCGCCAGGAGAG-3' & \multirow{2}{*}{61} \\
\hline & rev & 5'-TACAGGGTCAGCCGCTCCAG-3' & \\
\hline \multirow{2}{*}{ DAAO-NLS } & $\mathrm{fw}$ & $5^{\prime}$-CCTGAGACCCGCCAGGAGAG-3' & \multirow{2}{*}{61} \\
\hline & rev & 5'-GGATCGCTCTCCCTAGCTGC-3' & \\
\hline
\end{tabular}

\subsubsection{Antibody list}

Table 10: Antibodies used for BN-PAGE.

\begin{tabular}{|l|l|l|l|}
\hline Antibody & Raised in & Manufacturer & Article number \\
\hline anti-ATP5B & Rabbit & Gramsch & $4826 / 4$ \\
\hline anti-COXI & Rabbit & Gramsch & $2035 / 5$ \\
\hline anti-MCU & Rabbit & Sigma & HPA016480 \\
\hline anti-NDUFB8 & Rabbit & Gramsch & $3765 / 1$ \\
\hline
\end{tabular}




\begin{tabular}{|l|l|l|l|}
\hline anti-Rieske & Rabbit & Gramsch & $1512 / 6$ \\
\hline anti-VDAC3 & Rabbit & Gramsch & $151 / 7$ \\
\hline
\end{tabular}

\subsubsection{Plasmid list}

Table 11: Plasmids used for generating transgenic mouse models.

\begin{tabular}{|l|l|}
\hline Transgenic mouse model & Name of the plasmid \\
\hline DAAO-HyPer (NLS) & m-aMHC-Hyper-DAAO-NLS-hGHpolyA \\
\hline DAAO-HyPer (NES) & m-aMHC-Hyper-DAAO-NES-hGHpolyA \\
\hline
\end{tabular}

\subsubsection{Consumables}

Table 12: Consumables used in this work.

\begin{tabular}{|l|l|l|}
\hline Consumables & Manufacturer & Article number \\
\hline 6-well plates & Sarstedt & 83.392 \\
\hline $\begin{array}{l}\text { Black (transparent } \\
\text { bottom) 96-well plates }\end{array}$ & Falcon & 3538219 \\
\hline Black 96-well plates & Greiner bio-one & 655083 \\
\hline Coverslips & Th.Geyer & 41001124 \\
\hline Cryomolds & Tissue-Tek & 4566 \\
\hline Disposable scalpels & $\begin{array}{l}\text { Feather safety } \\
\text { razor Co., LTD }\end{array}$ & 02.001 .30 .021 \\
\hline Filter cloth & $\begin{array}{l}\text { Klein \& Wieler } \\
\text { oHG. }\end{array}$ & - \\
\hline Glass potters & Sartorius & BBI-8542708 \\
\hline Glass slides & Thermo scientific & 803711 \\
\hline Needle & $\begin{array}{l}\text { Self-made (In } \\
\text { workshop) }\end{array}$ & - \\
\hline O.C.T compound & Tissue-Tek & 4583 \\
\hline Petri dishes & Sarstedt & 833902500 \\
\hline PVDF membrane & $\begin{array}{l}\text { Immobilon (0.45 } \\
\text { Mm) }\end{array}$ & PVH00010 \\
\hline Single Edge Blades & GEM & Braun, Omnifix 40 \\
\hline Syringe & solo & \\
\hline
\end{tabular}




\begin{tabular}{|l|l|l|}
\hline $\begin{array}{l}\text { Thin filter paper } \\
(\varnothing=110 \mathrm{~mm})\end{array}$ & $\begin{array}{l}\text { Carl Roth } \mathrm{GmbH} \\
\text { GmbH }\end{array}$ & 431011 \\
\hline Threads & FST & $4-\mathrm{S}$ \\
\hline White 96-well plates & Greiner bio-one & 655083 \\
\hline
\end{tabular}

\subsubsection{Devices}

Table 13: List of devices used in this work.

\begin{tabular}{|c|c|c|}
\hline Devices & Manufacturer & Specifications \\
\hline $\begin{array}{l}\text { Blue native gel } \\
\text { electrophoresis } \\
\text { chamber }\end{array}$ & $\begin{array}{l}\text { Amersham } \\
\text { biosciences }\end{array}$ & $\begin{array}{l}\text { Hoefer SE600 } \\
\text { Ruby }\end{array}$ \\
\hline Bright field microscope & Motic & AE30 \\
\hline Bright field microscope & Nikon & Type 104 \\
\hline Centrifuge & Eppendorf & $5415 \mathrm{R}$ \\
\hline $\begin{array}{l}\text { Confocal laser } \\
\text { scanning microscope }\end{array}$ & $\begin{array}{l}\text { Carl Zeiss } \\
\text { Microscopy } \\
\text { GmbH } \\
\end{array}$ & LSM 510 \\
\hline Cryostat & Microm HM 560 & $\begin{array}{l}\text { Thermo Fisher } \\
\text { Scientific }\end{array}$ \\
\hline Hypoxia Chamber & Invivo400 & Baker Ruskinn \\
\hline $\begin{array}{l}\text { Inverted } \\
\text { epifluorescence } \\
\text { microscope }\end{array}$ & $\begin{array}{l}\text { Olympus-life } \\
\text { sciences }\end{array}$ & IX83 \\
\hline $\begin{array}{l}\text { Langendorff Perfusion } \\
\text { system }\end{array}$ & Ismatec & $\begin{array}{l}\text { Connected to } \\
\text { Julaba hot water } \\
\text { bath }\end{array}$ \\
\hline PCR cycler & Thermo scientific & $\begin{array}{l}\text { Arktik thermos } \\
\text { cycler }\end{array}$ \\
\hline Plate reader 1 & BMG labtech & CLARIOstar \\
\hline Plate reader 2 & Biorad & - \\
\hline Plate reader 3 & Berthold & Centro LB960 \\
\hline $\begin{array}{l}\text { Western blot transfer } \\
\text { system }\end{array}$ & $\begin{array}{l}\text { PeqLab } \\
\text { biotechnologie } \\
\text { GmbH }\end{array}$ & - \\
\hline
\end{tabular}




\subsubsection{Buffers}

Table 14: Components of buffers used and their concentrations.

Phosphate buffered saline (PBS)

\begin{tabular}{|l|l|}
\hline Components & Concentration \\
\hline $\mathrm{KCl}$ & $2.7 \mathrm{mM}$ \\
\hline $\mathrm{KH}_{2} \mathrm{HPO}_{4}$ & $1.8 \mathrm{mM}$ \\
\hline $\mathrm{Na}_{2} \mathrm{HPO}_{4}$ & $10 \mathrm{mM}$ \\
\hline $\mathrm{NaCl}$ & $137 \mathrm{mM}$ \\
\hline
\end{tabular}

Above components were dissolved in distilled $\mathrm{H}_{2} \mathrm{O}$ and $\mathrm{pH}$ was adjust to 7.4.

\section{Genotyping buffers}

Lysis buffer

\begin{tabular}{|l|l|}
\hline Components & Concentration \\
\hline $\mathrm{NaOH}$ & $25 \mathrm{mM}$ \\
\hline EDTA, pH 8.0 & $0.2 \mathrm{mM}$ \\
\hline
\end{tabular}

Above components were dissolved in distilled $\mathrm{H}_{2} \mathrm{O}$.

Neutralization buffer

\begin{tabular}{|l|l|}
\hline Components & Concentration \\
\hline Tris $/ \mathrm{HCl}, \mathrm{pH} 5.0$ & $40 \mathrm{mM}$ \\
\hline
\end{tabular}

Above components were dissolved in distilled $\mathrm{H}_{2} \mathrm{O}$.

TBE buffer (0.5X)

\begin{tabular}{|l|l|}
\hline Components & Concentration \\
\hline Tris & $45 \mathrm{mM}$ \\
\hline Boric acid & $45 \mathrm{mM}$ \\
\hline EDTA, pH 8.0 & $1 \mathrm{mM}$ \\
\hline
\end{tabular}

Above components were dissolved in distilled $\mathrm{H}_{2} \mathrm{O}$.

\section{Cardiomyocytes isolation buffers}

10x Stock perfusion buffer $(500 \mathrm{~mL})$

\begin{tabular}{|l|l|}
\hline Components & Concentration \\
\hline $\mathrm{NaCl}$ & $1.13 \mathrm{M}$ \\
\hline $\mathrm{KCl}$ & $47 \mathrm{mM}$ \\
\hline $\mathrm{KH}_{2} \mathrm{PO}_{4}$ & $6 \mathrm{mM}$ \\
\hline $\mathrm{Na}_{2} \mathrm{HPO}_{4}, 2 \mathrm{H}_{2} \mathrm{O}$ & $6 \mathrm{mM}$ \\
\hline
\end{tabular}




\begin{tabular}{|l|l|}
\hline $\mathrm{MgSO}_{4}, 7 \mathrm{H}_{2} \mathrm{O}$ & $12 \mathrm{mM}$ \\
\hline Phenol red & $0.32 \mathrm{mM}$ \\
\hline $\mathrm{NaHCO}_{3}$ & $120 \mathrm{mM}$ \\
\hline $\mathrm{KHCO}_{3}$ & $100 \mathrm{mM}$ \\
\hline HEPES & $100 \mathrm{mM}$ \\
\hline Taurine & $300 \mathrm{mM}$ \\
\hline
\end{tabular}

Above components were dissolved in final volume of $500 \mathrm{~mL}$ distilled $\mathrm{H}_{2} \mathrm{O}$, sterile filtrated and stored at $4^{\circ} \mathrm{C}$.

BDM solution (50 mL)

\begin{tabular}{|l|l|}
\hline Components & Concentration \\
\hline BDM & $500 \mathrm{mM}$ \\
\hline
\end{tabular}

BDM powder was dissolved in final volume of $50 \mathrm{~mL}$ distilled $\mathrm{H}_{2} \mathrm{O}$, sterile filtrated and stored at $-20^{\circ} \mathrm{C}$. The solution was warmed up before use.

BSA solution (50 $\mathrm{mL}$ )

\begin{tabular}{|l|l|}
\hline Components & Concentration \\
\hline BSA & $1.5 \mathrm{M}$ \\
\hline
\end{tabular}

BSA powder was dissolved in final volume of $50 \mathrm{~mL}$ distilled $\mathrm{H}_{2} \mathrm{O}$ and the solution was sterile filtrated. $800 \mu \mathrm{L}$ aliquots were prepared and aliquots were stored at $-20^{\circ} \mathrm{C}$.

Calcium chloride solution

\begin{tabular}{|l|l|}
\hline Components & Concentration \\
\hline $\mathrm{CaCl}_{2}, 2 \mathrm{H}_{2} \mathrm{O}$ & $100 \mathrm{mM}$ \\
\hline $\mathrm{CaCl}_{2}, 2 \mathrm{H}_{2} \mathrm{O}$ & $10 \mathrm{mM}$ \\
\hline
\end{tabular}

The Calcium chloride solutions were sterile filtrated and stored at $4^{\circ} \mathrm{C}$.

Trypsin solution

\begin{tabular}{|l|l|}
\hline Components & Concentration \\
\hline Trypsin & $2.50 \%$ \\
\hline
\end{tabular}

Trypsin solution was aliquot under sterile conditions to $200 \mu \mathrm{L}$ per aliquot and stored at $-20^{\circ} \mathrm{C}$.

Liberase solution

\begin{tabular}{|l|l|}
\hline Components & Concentration \\
\hline Liberase DH & - \\
\hline
\end{tabular}

Liberase enzyme powder was dissolved in $12 \mathrm{~mL}$ distilled $\mathrm{H}_{2} \mathrm{O}$ and reconstituted on ice for 20 min. The solution was aliquoted under sterile conditions to $150 \mu \mathrm{L}$ per aliquot and freezed it immediately in liquid nitrogen. The aliquots were stored at $-20^{\circ} \mathrm{C}$. 
1x Perfusion buffer $(500 \mathrm{~mL})$

\begin{tabular}{|l|l|}
\hline Components & Concentration \\
\hline 10x Perfusion buffer & $50 \mathrm{~mL}$ \\
\hline Glucose & $5.5 \mathrm{mM}$ \\
\hline BDM solution & $10 \mathrm{mM}$ \\
\hline
\end{tabular}

The above components were dissolved in final volume of $500 \mathrm{~mL}$ distilled $\mathrm{H}_{2} \mathrm{O}$. The buffer was aliquoted under sterile conditions to $35 \mathrm{~mL}$ per aliquot falcon tubes and stored at $-20^{\circ} \mathrm{C}$.

Digestion buffer $(30 \mathrm{~mL})$

\begin{tabular}{|l|l|}
\hline Components & Concentration \\
\hline $1 \times$ Perfusion buffer & $29.6 \mathrm{~mL}$ \\
\hline $\mathrm{CaCl}_{2}$ solution & $12.5 \mathrm{mM}$ \\
\hline Trypsin solution & $200 \mu \mathrm{L}$ \\
\hline Liberase solution & $300 \mu \mathrm{L}$ \\
\hline
\end{tabular}

Stopping buffer 1

\begin{tabular}{|l|l|}
\hline Components & Concentration \\
\hline $1 \times$ Perfusion buffer & $2.25 \mathrm{~mL}$ \\
\hline BSA solution & $150 \mathrm{mM}$ \\
\hline $\mathrm{CaCl}_{2}$ solution & $55.55 \mu \mathrm{M}$ \\
\hline
\end{tabular}

Stopping buffer 2

\begin{tabular}{|l|l|}
\hline Components & Concentration \\
\hline $1 \times$ Perfusion buffer & $9.5 \mathrm{~mL}$ \\
\hline BSA solution & $300 \mathrm{mM}$ \\
\hline $\mathrm{CaCl}_{2}$ solution & $166 \mu \mathrm{M}$ \\
\hline
\end{tabular}

Imaging buffer, $\mathrm{pH} 7.4$

\begin{tabular}{|l|l|}
\hline Components & Concentration \\
\hline $\mathrm{NaCl}$ & $144 \mathrm{mM}$ \\
\hline $\mathrm{KCl}$ & $5.4 \mathrm{mM}$ \\
\hline $\mathrm{MgCl}_{2}, 6 \mathrm{H}_{2} \mathrm{O}$ & $1 \mathrm{mM}$ \\
\hline $\mathrm{CaCl}_{2}$ & $1 \mathrm{mM}$ \\
\hline $\mathrm{HEPES}$ & $10 \mathrm{mM}$ \\
\hline
\end{tabular}


Diamide

\begin{tabular}{|l|l|}
\hline Components & Concentration \\
\hline Diamide & $100 \mu \mathrm{M}$ \\
\hline
\end{tabular}

Diamide powder is dissolved in imaging buffer and used at a final concentration of $100 \mu \mathrm{M}$ $\mathrm{H}_{2} \mathrm{O}_{2}$

\begin{tabular}{|l|l|}
\hline Components & Concentration \\
\hline $\mathrm{H} 2 \mathrm{O} 2$ & $100 \mu \mathrm{M}$ \\
\hline
\end{tabular}

$\mathrm{H}_{2} \mathrm{O}_{2}$ is dissolved in imaging buffer and used at a final concentration of $100 \mu \mathrm{M}$

DTT

\begin{tabular}{|l|l|}
\hline Components & Concentration \\
\hline DTT & $2 \mathrm{mM}$ \\
\hline
\end{tabular}

DTT powder is dissolved in imaging buffer and used at a final concentration of $2 \mathrm{mM}$

Mitochondria isolation buffer, $\mathrm{pH}-7.4$

\begin{tabular}{|l|l|}
\hline Components & Concentration \\
\hline HEPES & $20 \mathrm{mM}$ \\
\hline Mannitol & $220 \mathrm{mM}$ \\
\hline Sucrose & $70 \mathrm{mM}$ \\
\hline EDTA & $1 \mathrm{mM}$ \\
\hline PMSF (freshly added) & $0.5 \mathrm{mM}$ \\
\hline
\end{tabular}

High sucrose buffer, $\mathrm{pH} 7.4$

\begin{tabular}{|l|l|}
\hline Components & Concentration \\
\hline MOPS & $10 \mathrm{mM}$ \\
\hline Sucrose & $250 \mathrm{mM}$ \\
\hline EDTA & $1 \mathrm{mM}$ \\
\hline
\end{tabular}

Complex III activity assay buffer

Assay buffer

\begin{tabular}{|l|l|}
\hline Components & Concentration \\
\hline Potassium phosphate & $50 \mathrm{mM}$ \\
\hline $\begin{array}{l}\text { n-Decylubichinone- } \\
\text { beta-maltoside }\end{array}$ & $1 \mathrm{mM}$ \\
\hline Potassium cyanide & $1 \mathrm{mM}$ \\
\hline
\end{tabular}




\begin{tabular}{|l|l|}
\hline Rotenone & $2.5 \mu \mathrm{M}$ \\
\hline BSA & $0.10 \%$ \\
\hline
\end{tabular}

Above components were dissolved in $\mathrm{H}_{2} \mathrm{O}$ and equilibrated at $30^{\circ} \mathrm{C}$.

Reduced decylubichinone

\begin{tabular}{|l|l|}
\hline Components & Concentration \\
\hline $\begin{array}{l}\text { Decylubichinone in } \\
\text { ethanol }\end{array}$ & $10 \mathrm{mM}$ \\
\hline $\mathrm{KBH}_{4}$ & $5 \mathrm{mg}$ \\
\hline $\mathrm{HCl}$ in ethanol & $0.1 \mathrm{M}$ \\
\hline
\end{tabular}

Above components were mixed and the solution was vortex for 1-3 minutes until it becomes clear. $12 \mu \mathrm{l} 3 \mathrm{M} \mathrm{HCl}$ in EtOH was added to the solution and centrifuged for $30 \mathrm{sec}$. Supernatant was transferred into fresh tube. This is the reduced decylubichinone.

\section{Blue native gel electrophoresis buffers}

Mitochondria solubilization buffer

\begin{tabular}{|l|l|}
\hline Components & Concentration \\
\hline Digitonin & $1 \%$ \\
\hline Tris/HCl, pH 7.4 & $20 \mathrm{mM}$ \\
\hline EDTA, pH 8.0 & $0.1 \mathrm{mM}$ \\
\hline $\mathrm{NaCl}$ & $50 \mathrm{mM}$ \\
\hline Glycerol & $10 \%$ \\
\hline PMSF & $1 \mathrm{mM}$ \\
\hline
\end{tabular}

Above components were dissolved in distilled $\mathrm{H}_{2} \mathrm{O}$.

$3 x$ gel buffer

\begin{tabular}{|l|l|}
\hline Components & Concentration \\
\hline e-Amino n-caproic acid & $200 \mathrm{mM}$ \\
\hline Bis-Tris/HCl, pH 7.0 & $150 \mathrm{mM}$ \\
\hline
\end{tabular}

Acrylamide $(200 \mathrm{ml})$

\begin{tabular}{|l|l|}
\hline Components & Concentration \\
\hline Acrylamide & $96 \mathrm{~g}$ \\
\hline Bis-acrylamide & $3 \mathrm{~g}$ \\
\hline
\end{tabular}


Loading dye (10x, $10 \mathrm{ml})$

\begin{tabular}{|l|l|}
\hline Components & Concentration \\
\hline Coomassie blue G & $5 \%$ \\
\hline e-amino n-caproic acid & $500 \mathrm{mM}$ \\
\hline Bis-Tris, pH 7.0 & $100 \mathrm{mM}$ \\
\hline
\end{tabular}

Mix for 1 gel for BN-PAGE

\begin{tabular}{|l|l|l|l|l|l|l|l|l|l|}
\hline$\%$ & $\mathbf{4}$ & $\mathbf{5}$ & $\mathbf{6}$ & $\mathbf{8}$ & $\mathbf{1 0}$ & $\mathbf{1 3}$ & $\mathbf{1 6 . 5}$ & $\mathbf{2 0}$ & $\begin{array}{l}\text { Stack } \\
\text {-ing } \\
\text { gel }\end{array}$ \\
\hline $\begin{array}{l}3 \times \mathrm{gel} \\
\text { buffer }(\mathrm{mL})\end{array}$ & 3 & 3 & 3 & 3 & 3 & 3 & 3 & 3 & 2.5 \\
\hline $\begin{array}{l}\text { Acrylamide } \\
(\mathrm{mL})\end{array}$ & 0.73 & 0.91 & 1.07 & 1.46 & 1.82 & 2.35 & 3.05 & 3.75 & 0.6 \\
\hline $\begin{array}{l}\text { Glycerol } \\
(\mathrm{mL})\end{array}$ & - & - & - & - & 1.8 & 1.8 & 1.8 & 1.8 & - \\
\hline $\begin{array}{l}10 \% \text { APS } \\
(\mu \mathrm{l})\end{array}$ & 45 & 45 & 45 & 45 & 45 & 45 & 45 & 45 & 60 \\
\hline $\begin{array}{l}\text { TEMED } \\
(\mu \mathrm{l})\end{array}$ & 4.5 & 4.5 & 4.5 & 4.5 & 4.5 & 4.5 & 4.5 & 4.5 & 10 \\
\hline Water $(\mathrm{mL})$ & 5.228 & 5.014 & 4.888 & 4.507 & 2.347 & 1.817 & 1.117 & 0.417 & 4.367 \\
\hline
\end{tabular}

10x anode buffer

\begin{tabular}{|l|l|}
\hline Components & Concentration \\
\hline Bis-Tris $/ \mathrm{HCl}, \mathrm{pH}-7.0$ & $500 \mathrm{mM}$ \\
\hline
\end{tabular}

10x cathode buffer

\begin{tabular}{|l|l|}
\hline Components & Concentration \\
\hline Tricine, $\mathrm{pH} 7.0$ & $500 \mathrm{mM}$ \\
\hline Bis-Tris/HCl, pH 7.0 & $150 \mathrm{mM}$ \\
\hline Coomassie blue G & $0.20 \%$ \\
\hline
\end{tabular}

Western blot transfer buffer

\begin{tabular}{|l|l|}
\hline Components & Concentration \\
\hline Tris & $20 \mathrm{mM}$ \\
\hline Glycine & $150 \mathrm{mM}$ \\
\hline SDS & $0.02 \%$ \\
\hline Methanol & $20 \%$ \\
\hline
\end{tabular}


10x TBS

\begin{tabular}{|l|l|}
\hline Components & Concentration \\
\hline Tris/HCl, pH 7.5 & $200 \mathrm{mM}$ \\
\hline $\mathrm{NaCl}$ & $1.25 \mathrm{M}$ \\
\hline
\end{tabular}

1x TBST (100 ml, dissolved in $\left.\mathrm{H}_{2} \mathrm{O}\right)$

\begin{tabular}{|l|l|}
\hline Components & Concentration \\
\hline $10 x$ TBS & $10 \mathrm{ml}$ \\
\hline Tween 20 & $2 \mathrm{ml}$ \\
\hline
\end{tabular}

Gel staining buffer

\begin{tabular}{|l|l|}
\hline Components & Concentration \\
\hline Ethanol & $40 \%$ \\
\hline Acetic acid & $10 \%$ \\
\hline Coomassie blue G & $0.15 \%$ \\
\hline
\end{tabular}

Gel destaining buffer

\begin{tabular}{|l|l|}
\hline Components & Concentration \\
\hline Ethanol & $30 \%$ \\
\hline Acetic acid & $10 \%$ \\
\hline
\end{tabular}

\subsection{Methods}

\subsubsection{Mouse models}

\section{Grx1-roGFP2 mouse model}

A cardiomyocyte specific transgenic mouse model expressing the glutathione redox biosensor Grx1-roGFP2 was previously generated and characterized in the group of Cardiovascular Physiology, Göttingen by Dr. Lija Swain (Swain et al., 2016). Two independent mouse lines were created, expressing the biosensor in two different compartments namely in the cytoplasm and in the mitochondrial matrix of cardiomyocyte respectively. The biosensor consisted of a $\alpha-$ myosin heavy chain promoter driven cyto Grx1-roGFP2 or mito Grx1-roGFP2 construct with or without the mitochondrial targeting sequence of Neurospora crassa ATP synthase protein 9. The mammalian expression vector pLPCXGrx1-roGFP2, which contains the sequence for cyto Grx1-roGFP2 or mito Grx1-roGFP2 was cut with HindIII and Xhol enzymes. The mammalian expression vector $\alpha$-myosin heavy chain pmEpac1, which contains the $\alpha$-myosin heavy chain 
promoter, was digested with HindIII and Xhol. DH10B Escherichia coli were transformed with the digested cyto Grx1-roGFP2 or mito Grx1-roGFP2. Endotoxin-free plasmid kit was used for preparation of DNA. Linearized DNA was recovered from gels and purified. Transgenic mice were created by pronuclear injection of C57BL/6N mice (Jackson Laboratories).

Presence of the respective transgene in the offspring was confirmed through genotyping of mice biopsies by a standard polymerase chain reaction using the primers for roGFP resulting in a 500-bp fragment for cyto Grx1-roGFP2 and 710-bp for mito Grx1-roGFP2. Primers for the Phd2 gene were used as a control. Adult transgenic mice of age 8-12 weeks were used in the experiments

\section{DAAO-HyPer mouse model}

DAAO-HyPer is a fusion protein consisting of the enzyme D-amino acid oxidase (DAAO) and the $\mathrm{H}_{2} \mathrm{O}_{2}$ biosensor HyPer. The fusion protein serves the purpose of intracellular local production of $\mathrm{H}_{2} \mathrm{O}_{2}$ upon activation of the enzyme DAAO, followed by recording the amount of $\mathrm{H}_{2} \mathrm{O}_{2}$ produced by the fused probe HyPer. In this thesis, two DAAO-HyPer transgenic mouse models wherein the construct is localized to two different compartments in two independent mouse lines namely, by DAAO-HyPer nuclear export signal (NES) and by the DAAO-HyPer nuclear localization signal (NLS) were generated. m-aMHC-Hyper-DAAO-NES-hGHpolyA and m-aMHC-Hyper-DAAO-NLS-hGHpolyA were the plasmids used the generation of the mouse models. XL1- Blue bacterial cells were transformed with the plasmids by giving a heat shock followed by overnight incubation. The plasmids were further isolated and subsequently linearized with the restriction enzyme EcoRV. The linearized plasmid was purified by a PCR purification kit (NEB Monarch). The purified DNA was diluted in TE buffer to reach a final concentration of 395 and $402 \mathrm{ng} / \mu \mathrm{l}$ each of the DAAO-HyPer NES and NLS respectively. Transgenic mice were further generated by pronuclear blastocyst injection of C57BL/6N mice (Jackson Laboratories) with the respective plasmids. The injection was performed by the Core Facility of the Max-Planck Institute for Experimental Medicine, Göttingen. We obtained 4 founder lines for the DAAO-HyPer NES and 5 for the DAAO-HyPer NLS. The transgenic mice of all the founder lines were further characterized for the presence of the transgene. 8-12 weeks old adult males and females were used for the characterization.

\section{Echocardiography}

Echocardiography is a method used to examine the heart function and geometry. It is a noninvasive method which works on the principle of ultrasound. Echocardiography was performed by Dr. Aline Jatho (Institute of Cardiovascular Physiology, Göttingen). Echocardiography was done on the Grx1-roGFP2 young and old mice in order to perform aging studies as well on the NLS DAAO HyPer mice. Each mouse was anesthetized by $1 \%$ isoflurane. At the beginning, 
the machine was set to B-mode to record at least 10 cardiac cycles to observe the long and the short axis of the heart. The device was then turned to M-mode in order to measure the heart beat with a better resolution. At this step again 10 cardiac cycles were measured in the short axis.

Different parameters were recorded such as fractional area shortening (FAS), ejection fraction $(E F)$, area of lumen during systole (Area (s)) and diastole (Area (d)), thickness of posterior and anterior walls, length of long and short axis of the heart ( $L(s)$ and $L(d))$.

The EF is defined as the volume of blood distributed with one heartbeat. The following formula was used to calculate EF (\%):

$\mathrm{EF}(\%)=\frac{\operatorname{Vol}(\mathrm{d})-\operatorname{Vol}(\mathrm{s})}{\operatorname{Vol}(\mathrm{d})} * 100$,

Where $\mathrm{Vol}(\mathrm{d})$ and $\mathrm{Vol}(\mathrm{s})$ stands for volume of left ventricles during diastole and systole respectively. These parameters were calculated using the formula,

$\operatorname{Vol}(d)=\left(\frac{5}{6}\right) *(\operatorname{Area}(d) * L(d))$

$\operatorname{Vol}(\mathrm{s})=\left(\frac{5}{6}\right) *(\operatorname{Area}(\mathrm{s}) * \mathrm{~L}(\mathrm{~s}))$

Fractional area shortening (FAS \%) is the fraction of the area of diastole which is lost in the systole. The formula to calculate the FAS (\%) is:

FAS $(\%)=\left(\frac{\operatorname{Area}(\mathrm{d})-\operatorname{Area}(\mathrm{s})}{\operatorname{Area}(\mathrm{d})}\right) * 100$

\section{Genotyping}

Tail biopsies were collected from 3-4 weeks old mice offsprings. In order to isolate genomic DNA, at first the biopsies were incubated at $95^{\circ} \mathrm{C}$ in $75 \mu$ lysis buffer for 60 minutes. $75 \mu \mathrm{l}$ of neutralization buffer was added to stop the lysis and incubated at $4^{\circ} \mathrm{C}$ for 10 minutes. Presence of the respective transgene in the corresponding mouse model was confirmed by a standard PCR reaction. PCR mix was prepared by adding $12.5 \mu \mathrm{l} \mathrm{PCR}$ master mix (2x) to $5 \mu \mathrm{l}$ of the DNA containing lysate. $0.1 \mathrm{pmol} / \mu \mathrm{l}$ of forward and reverse primer for the respective mouse line were added to the above mix. The final volume of the PCR mix was made up to $25 \mu \mathrm{l}$ by adding remaining volume of $\mathrm{H}_{2} \mathrm{O}$. Following were the temperature settings for the respective PCR reactions: 


\begin{tabular}{|l|l|l|l|l|l|l|}
\hline \multirow{2}{*}{ Reaction } & \multicolumn{2}{|l|}{ Temperature $\left.^{\circ}{ }^{\circ} \mathbf{C}\right)$} & \multicolumn{2}{l|}{ t(sec)/cycle } \\
\cline { 2 - 8 } & roGFP2 & PHD2 & $\begin{array}{l}\text { DAAO- } \\
\text { NES }\end{array}$ & $\begin{array}{l}\text { DAAO- } \\
\text { NLS }\end{array}$ & \multicolumn{2}{|c|}{} \\
\hline Separation 1 & 95 & 95 & 94 & 94 & 180 & 30 \\
\hline Separation 2 & 95 & 95 & 94 & 94 & 30 & 35x \\
\hline Annealing & $\mathbf{5 8}$ & $\mathbf{5 8}$ & $\mathbf{6 1}$ & $\mathbf{6 1}$ & 30 & \\
\hline Elongation & 72 & 72 & 72 & 72 & 90 & 600 \\
\hline Final elongation & 72 & 72 & 72 & 72 & & \\
\hline
\end{tabular}

Table 15: PCR conditions for genotyping.

A 2\% agarose gel was used to run the above prepared PCR reaction mix. The PCR product was mixed with $5 \mu$ l Orange $G$ dye and allowed to run on a gel in $0.5 x$ TBE buffer along with Roti safe gel stain and $1 \mathrm{~kb}$ base pair ladder. The expected PCR product in the case of cyto Grx1-roGFP2 mouse biopsy was at $500 \mathrm{bp}$, whereas in the case of mito Grx1-roGFP2 the expected PCR product was at $710 \mathrm{bp}$. The PCR product for both DAO-NES and DAO-NLS was around $280 \mathrm{bp}$.

\section{Extraction of Mouse heart}

The mouse was anesthetized with isoflurane and was sacrificed by cervical dislocation. The area over the chest was disinfected by $70 \%$ ethanol and the fur was removed from this part. Further, the thoracic cavity was opened up and the pericardial sac was distorted to expose the heart. The heart was gently extracted keeping intact the aortic valve along with the heart.

\subsubsection{Isolated cardiomyocytes and associated redox analysis}

\section{Isolation of cardiomyocytes}

As soon as the heart was extracted from a transgenic or a wild type mouse, it was immediately transferred to ice cold PBS. Under a pre-set Nikon Type104 inverted microscope with a needle fasted into the syringe, the aorta of the heart was identified by gently pushing the heart. The walls of the aorta were grabbed with two fine forceps and the aorta was slipped on to the needle until some resistance was felt, without penetrating the heart. The tread loops were used to fasten the aorta tightly around the needle. The whole procedure from extracting the heart to hanging the aorta of the heart was done as quickly as possible. The hanged heart along with the needle was then fixed onto the perfusion system which was connected to the pre warmed $\left(37^{\circ} \mathrm{C}\right)$ water bath. The heart was initially perfused with a pre warmed $1 \mathrm{x}$ perfusion buffer for 3 min with a speed of $3.5 \mathrm{~mL} / \mathrm{min}$. The first few drops of blood dropping from the heart, ventricles turning pale and heart blowing a bit, confirmed that the heart was hung up with the correct blood vessel which in this case should be aorta. During the first perfusion time, $2.5 \%$ trypsin 
and liberase enzymes were thawed and the digestion buffer was prepared for the next perfusion step. The heart was then perfused with the freshly prepared digestion buffer saving a portion of $2.5 \mathrm{~mL}$ digestion buffer for further use. The perfusion was allowed to run approximately for 8 minutes until the whole digestion buffer was used up. Throughout the perfusion time, it was taken care that there were no air bubbles going through the heart while perfusing. The heart was then pulled out from the needle and put into the earlier saved $2.5 \mathrm{~mL}$ digestion buffer. The heart was minced with fine scissors for $30 \mathrm{sec}$, followed by adding the stopping buffer 1 into the minced heart solution. Now the mixture was digested with the help of up and down action of a syringe for 2.5 minutes. The minced solution was allowed to filter out through a filter cloth. In this step, some drops of the solution were used to check for the presence of viable cardiomyocytes under a bright field microscope. The cardiomyocytes were allowed to settle down in the falcon tube for 10 minutes and then carefully the supernatant was discarded. The pelleted cardiomyocytes were resuspended in stopping buffer 2 followed by beginning of the recalcification steps. Table 16 indicates the amounts of calcium chloride solution added followed by gentle shaking and allowing the cells to adapt for $4 \mathrm{~min}$ in between the recalcification steps.

\begin{tabular}{|l|l|l|}
\hline $\begin{array}{l}\mathrm{CaCl}_{2} \text { stock } \\
\text { concentration }\end{array}$ & $\begin{array}{l}\text { Volume added to } \\
\text { cardiomyocytes } \\
\text { from the stock }\end{array}$ & $\begin{array}{l}\text { Final } \\
\text { concentration }\end{array}$ \\
\hline $10 \mathrm{mM}$ & $50 \mu \mathrm{l}$ & $62 \mu \mathrm{M}$ \\
\hline $10 \mathrm{mM}$ & $50 \mu \mathrm{l}$ & $112 \mu \mathrm{M}$ \\
\hline $10 \mathrm{mM}$ & $100 \mu \mathrm{l}$ & $212 \mu \mathrm{M}$ \\
\hline $100 \mathrm{mM}$ & $30 \mu \mathrm{l}$ & $500 \mu \mathrm{M}$ \\
\hline $100 \mathrm{mM}$ & $50 \mu \mathrm{l}$ & $1000 \mu \mathrm{M}$ \\
\hline
\end{tabular}

Table 16: Amounts of calcium chloride added during the recalcification step.

The cardiomyocytes were then seeded on to the coverslips that were pre-coated with laminin. The cardiomyocytes were then incubated in an incubator $\left(37^{\circ} \mathrm{C}, 5 \% \mathrm{CO}_{2}\right.$ and $\left.20.9 \% \mathrm{O}_{2}\right)$. The cells were allowed to settle down and get attached onto the laminin before using them for any further experiments.

\section{Glutathione redox potential measurements of isolated cardiomyocytes}

$E_{G S H}$ of isolated cardiomyocytes were performed using an inverted fluorescence microscope IX83 (Olympus) and Cell sense software. Coverslips that were pre-coated with laminin containing the isolated cardiomyocytes were incubated for at least 45 minutes in the incubator before use. It was made sure that the microscope was pre-set to $37^{\circ} \mathrm{C}, 5 \% \mathrm{CO}_{2}$ and $20.9 \% \mathrm{O}_{2}$ before starting the experiment. Perfusion buffer used for incubating cardiomyocytes was first replaced by $900 \mu \mathrm{l}$ imaging buffer. Coverslip having the cardiomyocytes with imaging buffer 
was adjusted onto the microscope chamber and the cells were visualized under $20 \mathrm{x}$ magnification. The roGFP sensor was excited at 405 and $488 \mathrm{~nm}$ wavelengths and the emitted light was detected at $510 \mathrm{~nm}$. The exposure time was set to $10 \mathrm{~ms}$ and the images were acquired every $5 \mathrm{~s}$. Once a stable baseline of fluorescence intensities was observed, at approximately $50 \mathrm{~s}, 100 \mu \mathrm{l}$ of the desired oxidizing agent (100 $\mu \mathrm{M} \mathrm{H}_{2} \mathrm{O}_{2}$ or $100 \mu \mathrm{M}$ Diamide) or reducing agent (2 mM DTT) was added to the cells. The measurements were allowed to run for roughly $300 \mathrm{sec}$, until a plateau of the fluorescence intensities was obtained. The changes in the fluorescence intensities were recorded after addition of each of the oxidizing and the reducing agent at the respective excitation wavelengths. The following Nernst equation was used in order to calculate the $E_{\mathrm{GSH}}$ of the corresponding cardiomyocytes:

$$
E_{G S H}=E_{\text {roGFP2 }}^{\circ}-\frac{R T}{2 F} \ln \left(\frac{1-O x D_{\text {roGFP2 }}}{O x D_{\text {roGFP2 }}}\right)
$$

Where,

$E^{\circ}{ }_{\text {roGFP2 }}=-280 \mathrm{mV}$

$R$ is the gas constant $\left(8.315 \mathrm{~J} \mathrm{~K}^{-1} \mathrm{~mol}^{-1}\right)$,

$T$ is the absolute temperature $(298.15 \mathrm{~K})$,

$F$ the Faraday constant $\left(96,485 \mathrm{C} \mathrm{mol}^{-1}\right)$, and

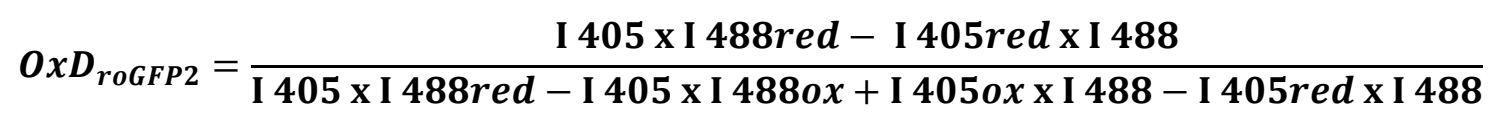

Where, $i 405_{\text {red, }}$ i405 ${ }_{\text {ox }}, i 488_{\text {red, }}$ and $1488_{o x}$ stands for the fluorescence intensities at the indicated wavelength and redox state for the roGFP2 molecules.

\section{Redox measurements of DAAO-HyPer cardiomyocytes}

NLS and NES DAAO-HyPer mouse models consist of a DAAO-HyPer biosensor which is a fusion protein present in the respective organelle of the cardiomyocytes. The enzyme DAAO can be first activated by its substrates like D-alanine. Upon activation, DAAO can produce $\mathrm{H}_{2} \mathrm{O}_{2}$ endogenously in the cardiomyocytes. The produced $\mathrm{H}_{2} \mathrm{O}_{2}$ can be measured by the fused HyPer probe.

Cardiomyocytes from transgenic NLS or NES DAAO-HyPer mice were isolated by Langendorff's perfusion system. The cardiomyocytes were plated on the coverslips pre-coated with laminin and allowed to settle down for at least 45 minutes in a cell culture incubator. Once the cells were ready for imaging, the live imaging was performed on an inverted fluorescence microscope IX83 (Olympus) using the Cell sense software. The perfusion buffer in which the cardiomyocytes were initially incubated was first replaced by $900 \mu \mathrm{l}$ imaging buffer. A 10x 
magnification was used for the visualization of the cells. Cardiomyocytes were excited with the excitation wavelengths of HyPer probe at 420 and $500 \mathrm{~nm}$ and the emitted light was recorded at $510 \mathrm{~nm}$. The light intensity was set to $11 \%$ and the exposure time was set to $1000 \mathrm{~ms}$. Each image was recorded at an interval of $30 \mathrm{~s}$. Approximately after 4 minutes, $100 \mu \mathrm{l}$ of of D-alanine of various concentrations was added to the medium covering the cells. Once the DAAO was activated by D-alanine, there were some changes in the fluorescence intensities observed at 420 and $500 \mathrm{~nm}$ HyPer probe excitation wavelengths. Upon oxidation, the fluorescence intensities at $420 \mathrm{~nm}$ wavelength increased overtime and the fluorescence intensities at 500 $\mathrm{nm}$ decreased overtime. These changes were recorded until a plateau phase was achieved. At the end of the plateau phase $100 \mu \mathrm{M}$ of $\mathrm{H}_{2} \mathrm{O}_{2}$ was added in order to achieve full oxidation of the sensor. The entire measurement procedure was repeated with various concentrations of D-alanine starting form $10 \mathrm{mM}, 8 \mathrm{mM}, 6 \mathrm{mM}, 4 \mathrm{mM}$ to $3 \mathrm{mM}$ of D-alanine. L-alanine was also used as a control for the enzyme DAAO activation. Same concentrations of L-alanine were also used to record the measurements over time with the isolated cardiomyocytes.

\subsubsection{Preparation of cardiac tissue sections and associated analysis}

The heart was harvested from a transgenic mito Grx1-roGFP2 mouse, and was immediately transferred to ice cold PBS. The heart was gently pushed to remove excess blood and the atria were discarded leaving behind the intact ventricular part of the heart. Now, the bottom half of the heart was placed into the cryomold where the apex was facing upwards. The entire tissue in the cryomold was covered by O.C.T. compound and was immediately frozen on a pre-cooled aluminium block immersed in liquid nitrogen. This cryomolds were then stored at $-80^{\circ} \mathrm{C}$. In order to prepare cryosections from this, the cryostat was pre-cooled to $-20^{\circ} \mathrm{C}$ and the dissecting blade to $-25^{\circ} \mathrm{C}$. Cryomolds were allowed to thaw in the cryostat for 45 minutes followed by carefully slicing the tissue with a thickness of $14 \mu \mathrm{m}$. Subsequently, each slice for different conditions was placed on a glass slide respectively. Hence forth, all the steps were done on an ice cold aluminium block. Each slice was treated with either $50 \mu \mathrm{l}$ of $1 \mathrm{mM}$ Diamide, 20 mM DTT, 50 mM NEM, 1x PBS followed by incubation of 10 minutes on ice. The reagents were then wiped off with a fresh filter paper. Now each slice was treated with 50 mM NEM and incubated for 10 minutes. NEM was wiped gently from all slices and further the slices were fixed with ice cold 4\% PFA prepared in PBS containing To-Pro-3 lodide (1:1000) nuclear stain for 15 minutes. The slices were then gently wiped and washed with PBS, mounted on the coverslip with Mowiol. Once dried, the slides were then imaged with a LSM 510 laser scanning microscope (Carl Zeiss). The Grx-roGFP2 sensor was excited at 405 and $488 \mathrm{~nm}$ and the emission filter was set as $510 \mathrm{~nm}$. The images were then analysed by the ImageJ software. 


\subsubsection{Isolated mitochondria and associated analysis}

\section{Isolation of mitochondria from mouse heart in normoxia and hypoxia}

The heart was extracted from the mouse and was immediately transferred to ice cold PBS. It was gently rinsed to get rid of the excess blood. Atria were discarded and further the ventricles of the heart were used for isolating the mitochondria. All the steps of isolation were performed on ice. Ventricles were cut into small pieces and homogenized 25 times in a glass potter with the isolation buffer. The homogenate was centrifuged at $800 \mathrm{~g}$ for 10 minutes. Supernatant was transferred into a fresh tube, saved for further steps and the pellet was again homogenized 15 times with $1 \mathrm{ml}$ of isolation buffer. The homogenate was centrifuged at $800 \mathrm{~g}$ for 10 minutes. The supernatant was pooled along with the earlier saved supernatant and was further centrifuged at $10,000 \mathrm{~g}$ for 30 minutes. Now, the supernatant was discarded and the pellet was washed in $1.5 \mathrm{ml}$ of the isolation buffer at $10,000 \mathrm{~g}$ for 10 minutes. The final pellet was resuspended into $50-60 \mu \mathrm{l}$ of the buffer depending on quantity of the pellet. This pellet was used for protein estimation by Bradford protein estimation assay (Bio-Rad, protein assay dye reagent concentrate). The final protein concentration of the isolated mitochondria was set to 5 $\mu \mathrm{g} / \mu \mathrm{l}$.

The entire process of isolation was done simultaneously in normoxic conditions $\left(20.9 \% \mathrm{O}_{2}\right.$ ) and in hypoxic conditions $\left(0.1 \%, 3 \%, 5 \%\right.$ or $10 \% \mathrm{O}_{2}$, in invivo ${ }_{2} 400$ hypoxia workstation). Special care was taken during isolation of the mitochondria in hypoxia in order to maintain the hypoxic conditions throughout including equilibrating all the equipment used for isolation (pipettes, tips, Eppendorf tubes, glass potter, buffers etc.) to the hypoxic environment by incubating them overnight in the required hypoxic concentration, usage of airtight Eppendorf tubes while transferring samples for centrifugation steps and quick transfer of the centrifuged product into the hypoxia box. Once the isolation was finished both in normoxia and hypoxia, the isolated mitochondria were allowed to settle down on ice for at least one hour before using them for any further experiment.

\section{Bradford protein estimation assay}

The protein concentration of the isolated mitochondria was measured by Bradford protein estimation reagent. The reagent, when bound by a protein, has a shift in absorbance from 470 $\mathrm{nm}$ to $595 \mathrm{~nm}$. This shift in absorbance was recorded with a micro plate reader. A standard curve was generated through an increasing concentration of BSA from $0.5 \mu \mathrm{g} / \mu \mathrm{l}$ to $4 \mu \mathrm{g} / \mu \mathrm{l}$. Samples of unknown concentration were diluted to 1:10 out of which $1 \mu$ from each sample was used for the assay. $200 \mu \mathrm{l}$ of the 1:5 diluted reagent was added to each standard and the 
samples. The shift in the absorbance of the BSA standards and the samples was recorded for estimating the unknown concentration of the samples of isolated mitochondria.

\section{Redox measurements of isolated mitochondria}

Mitochondria were isolated simultaneously in normoxic and hypoxic conditions from cardiomyocyte specific Grx1-roGFP2 transgenic biosensor mice. The heart was divided into two parts so as to isolate in presence or in absence of $50 \mathrm{mM} \mathrm{N}$-Ethylmaleimide (NEM) during the entire isolation process with each of the part respectively. Isolated mitochondria with and without NEM were diluted in high sucrose buffer \pm NEM respectively to get the final concentration of mitochondria to $0.0317 \mu \mathrm{g} / \mu \mathrm{l}$. The change in the fluorescence intensities of mitochondria upon treatment with $100 \mu \mathrm{M}$ diamide, 2 mM DTT and a control condition without any treatment respectively was measured in the CLARIOstar microplate reader. The readings were recorded at an excitation of 405 and $488 \mathrm{~nm}$ wavelengths. It was made sure that the plate reader was set to the required oxygen concentration before starting the measurements. The mitochondria isolated in hypoxia were transported to the plate reader in an airtight container.

\section{Measurement of superoxide anion $\left(\mathrm{O}_{2}^{-}\right)$levels}

Mitochondria isolated in normoxia and hypoxia were diluted to the final concentration of $25 \mu \mathrm{g}$ in high sucrose buffer with or without $2.5 \mathrm{mM}$ succinate/0.5 mM ADP respectively. MitoSOX Red Mitochondrial Superoxide Indicator (Invitrogen, M36008) was added at a concentration of $5 \mu \mathrm{M}$ to each of the conditions and superoxide anions levels were recorded in the CLARIOstar microplate reader at the required oxygen concentration. The MitoSOX dye was excited at 510 $\mathrm{nm}$ and emission filter was set to $580 \mathrm{~nm}$. The measurement was allowed to run for 20 minutes and the average of the plateau phase of last 10 minutes was considered for analysis. All samples were pipetted in triplicates.

\section{Amplex Red assay}

Hydrogen peroxide concentration in the isolated mitochondria was measured by the Amplex Ultrared dye. Assay components consisted of $50 \mu \mathrm{M}$ of Amplex Red dye, $0.5 \mathrm{U} / \mathrm{ml}$ of HRP and $100 \mathrm{U} / \mathrm{ml}$ of superoxide dismutase (SOD) dissolved in high sucrose buffer. Mitochondria isolated in normoxia and hypoxia were diluted to the final concentration of $25 \mu \mathrm{g}$ per condition in a high sucrose buffer with or without $2.5 \mathrm{mM}$ succinate/0.5 mM ADP. A solution containing mitochondria solution and assay buffer in a 1:1 ratio was prepared in a black 96 -well plate (with transparent bottom) for each sample. The Amplex Red dye reacts with $\mathrm{H}_{2} \mathrm{O}_{2}$ to form the fluorescent product Resorufin which can be measured at excitation wavelength of $535 \mathrm{~nm}$ and emission of $590 \mathrm{~nm}$. The measurements were recorded for 20 minutes in a CLARIOstar plate 
reader at the required oxygen concentrations. Average reading of last 10 minutes plateau phase was used for actual analysis. All samples were pipetted in triplicates.

\section{Complex III activity assay}

Complex III activity assay was done by Dr. Jan Dudek from the Institute of Cellular Biochemistry, Göttingen. The assay involves the capacity of the ubiquinol cytochrome c reductase (complex III) to reduce cytochrome $\mathrm{c}$ by a reduced Decylubichinone as an electron donor. The activity can be measured spectrophotometrically at $550 \mathrm{~nm} .1 \mathrm{ml}$ complex III buffer was taken into a plastic disposable cuvette. $10 \mu \mathrm{l}$ of $10 \mathrm{mM}$ reduced Decylubichinone was added to the buffer and mixed well. $7.5 \mu$ of $2 \mathrm{mM}$ Cytochrome c $\left(25 \mathrm{mg} / \mathrm{ml}\right.$ in $\left.\mathrm{H}_{2} \mathrm{O}\right)$ was added to the above mixture. The measurement was started and after 30 seconds $10-50 \mu \mathrm{g}$ mitochondria, isolated in $20 \% \mathrm{O}_{2}$ and $0.1 \% \mathrm{O}_{2}$ respectively, were added to the cuvette. The measurement was continued for an average of 5 minutes. A blank measurement as a control was also included which contains only complex III buffer and no other components like cytochrome c, reduced Decylubichinone and mitochondria. Another control included was Antimycin $\mathrm{A}(10 \mathrm{mM})$ treated isolated mitochondria to exclude that the measurements involves a non-enzymatic reduction of cytochrome c. All samples were pipetted in triplicates.

\section{ATP production}

Mitochondria isolated in normoxia and hypoxia were diluted to the final concentration of $25 \mu \mathrm{g}$ in high sucrose buffer with or without $2.5 \mathrm{mM}$ succinate/0.5 mM ADP respectively and a condition with $2.5 \mathrm{mM}$ succinate/0.5 mM ADP + $10 \mu \mathrm{M} \mathrm{CaCl}_{2}$. Mitochondria were incubated in the respective buffers for 20 minutes. These mitochondria were then centrifuged at 10,000 rpm for 10 minutes. Supernatant was used for the ATP measurements. ATP standards $(0,0.001$, $0.01,0.1,1,10 \mu \mathrm{M})$ were used for calorimetric detection of the concentration of the samples. To each of the conditions including the standards, Celltiter-Glo luminescent cell viability reagent was added in a 1:1 dilution to the standard or the sample respectively. The reaction was incubated with gentle shaking of the plate for 30 minutes. The measurements were done in a calorimetric plate reader. All samples were pipetted in triplicates.

\section{Blue native gel electrophoresis}

Cardiac mitochondria were isolated in normoxia and hypoxia simultaneously. The mitochondria (50 $\mathrm{\mu g}$ per condition) were solubilized in the corresponding oxygen concentrations by resuspending them in $50 \mu \mathrm{l}$ of solubilisation buffer and were subsequently incubated on ice for 20 minutes. The solubilized mitochondria were centrifuged at $14,000 \mathrm{rpm}, 4^{\circ} \mathrm{C}$ for 5 minutes. The supernatant was transferred to a new precooled Eppendorf tube and mixed with $5 \mu$ l of loading 
dye. The sample was shortly centrifuged at $14,000 \mathrm{rpm}, 4^{\circ} \mathrm{C}$ for 2 minutes to get rid of cellular debris. The blue native gels were prepared well in advance and were kept ready for the mitochondrial extracts to be loaded. The ingredients for the separating and the stacking gel are given in Table 14. In this case, a 6-13\% separating gel was prepared. Once the mitochondrial extracts were ready and loaded onto the gel, cathode buffer was overlaid on top of the samples. The gel chamber containing the anode buffer was precooled to $4^{\circ} \mathrm{C}$ and the temperature was maintained throughout the run. The gel was initially set to run at $100 \mathrm{~V}$. Later the voltage was increased to $600 \mathrm{~V}$. Once the Coomassie blue dye was entering almost half way through the gel, the cathode buffer was replaced by fresh cathode buffer containing no coomassie. For overnight run from this stage, the voltage can be set at 60-70 V. Once the blue front of coomassie dye ran out of the gel, the run was stopped. The gel was incubated in SDS PAGE running buffer for 10 minutes prior to the western blot transfer.

The gel was then further used for the western blotting. PVDF membrane was used for blotting the gel. The membrane was briefly rinsed in methanol. Whatman filter papers were completely soaked in the transfer buffer. On the surface of the semidry blotting system, a transfer sandwich was prepared including a first layer of 3 whatman papers, the second layer containing the preactivated PVDF membrane followed by the gel and the last layer of 3 whatman filter papers. At each stage, it was made sure that there are no bubbles formed during the process. The transfer system was set and the run was started at $220 \mathrm{~mA}$ for 1 hour. Once the transfer was over, the membrane was stained and distained in order to confirm that the proteins were transferred. Later, the membrane was prepared for confirming the presence of the protein of interest. Table 17 summarizes the conditions used for the respective protein of interests. The membranes were incubated in blocking buffer for 60 minutes following overnight incubation with the primary antibody. To get rid of unbound primary antibody, the membranes were washed 3 times, 10 minutes each with TBST before and after addition of the respective HRP coupled secondary antibody. Subsequently the membranes were incubated in the enhanced chemiluminescence (ECL) solution which consists of the substrate for the HRP. The membranes were further developed on an X-ray film with varied exposure times depending on the protein of interest. 


\begin{tabular}{|l|l|l|l|}
\hline Protein of interest & Blocking solution & Primary antibody & $\begin{array}{l}\text { Secondary } \\
\text { antibody }\end{array}$ \\
\hline ATP5B & $\begin{array}{l}10 \% \text { skimmed milk } \\
\text { in TBST }\end{array}$ & $\begin{array}{l}1: 10000 \text { in TBST } \\
\text { COXI }\end{array}$ & $\begin{array}{l}1: 10000,10 \% \\
\text { skimmed milk in } \\
\text { TBST (Anti-rabbit) }\end{array}$ \\
\hline MCU & $\begin{array}{l}10 \% \text { skimmed milk } \\
\text { in TBST }\end{array}$ & $1: 1000$ in TBST & $\begin{array}{l}1: 10000,10 \% \\
\text { skimmed milk in } \\
\text { TBST (Anti-rabbit) }\end{array}$ \\
\hline NDUFB8 & $\begin{array}{l}10 \% \text { skimmed milk } \\
\text { in TBST }\end{array}$ & $1: 750$ in TBST & $\begin{array}{l}1: 5000,10 \% \\
\text { skimmed milk in } \\
\text { TBST (Anti-rabbit) }\end{array}$ \\
\hline Rieske & $\begin{array}{l}10 \% \text { skimmed milk } \\
\text { in TBST }\end{array}$ & $1: 1000$ in TBST & $\begin{array}{l}1: 10000,10 \% \\
\text { skimmed milk in } \\
\text { TBST (Anti-rabbit) }\end{array}$ \\
\hline VDAC3 & $\begin{array}{l}10 \% \text { skimmed milk } \\
\text { in TBST }\end{array}$ & $\begin{array}{l}1: 10000,10 \% \\
\text { skimmed milk in } \\
\text { TBST (Anti-rabbit) }\end{array}$ \\
\hline
\end{tabular}

Table 17: Conditions of each antibody used for detecting the protein of interest. 


\section{Results}

The overall goal of the thesis was to characterize redox-dependent processes in cardiomyocytes by making use of genetically encoded biosensors. Genetically encoded biosensors in combination with transgenic mouse technology allow to precisely determining redox processes in defined primary cell types and subcellular compartments. To this end I included in my studies the already established cardiomyocyte-specific Grx1-roGFP2 transgenic mice. These mice were used in the past to unravel that the $E_{\mathrm{GSH}}$ differ in the cytosol versus mitochondrial matrix in cardiomyocytes.

As a follow up, I analyzed in the first part of the thesis, if ageing affects the compartmentalization of the $E_{\mathrm{GSH}}$ in cardiomyocytes. In these studies I analyzed the $E_{\mathrm{GSH}}$ in the mitochondrial matrix in intact cardiomyocytes, in cryosections, and isolated mitochondria. Since I observed a significant difference in the mitochondrial $E_{G S H}$ if analyzed in situ or in isolated mitochondria, I studied the underlying mechanism in the second part of the thesis.

Grx1-roGFP2 allows determining the $E_{G S H}$ and thus the output of endogenous changes in redox signaling. Manipulation of endogenous redox signaling in cardiomyocytes was done in the past mostly by adding $\mathrm{H}_{2} \mathrm{O}_{2}$ to cells. This method, however, precludes precise analysis of dose-dependent effects as well as consequences for specific subcellular compartments. Therefore I aimed in the third part of my thesis to generate cardiomyocyte-specific transgenic mice, in which the DAAO-HyPer fusion protein is expressed in different subcellular compartments.

\subsection{Ageing affects the compartmentalization of $E_{G S H}$ in cardiomyocytes}

\subsubsection{Generation of Grx1-roGFP2 biosensor mouse models}

The main objective of the first part of my thesis was to address the phenomenon of $E_{G S H}$ compartmentalization observed in cardiomyocytes. For this purpose, I utilized the Grx1roGFP2 mouse models which were previously generated and characterized in the Institute of Cardiovascular Physiology by Dr. Lija Swain (Swain et al., 2016). The established mouse models enable to perform real time visualization of $E_{G S H}$ in the cytoplasm (cyto) and the mitochondrial matrix (mito) of the cardiomyocytes. A scheme of the plasmids used for generating the transgenic mice is shown in Fig. 9. Cardiomyocyte-specific expression of the biosensor is achieved by fusing the $\alpha-M H C$ promoter to the Grx1-roGFP2 coding region in the cyto as well as the mito mouse model. In the cyto mouse model the biosensor is non-targeted to a specific compartment and thus localized mostly to the cytosol (Fig. 9a). In case of the mito 
mouse model, the compartment specific expression is permitted via the Neurospora crassa ATP synthase protein 9 (atp9) signal sequence (Fig. 9b).

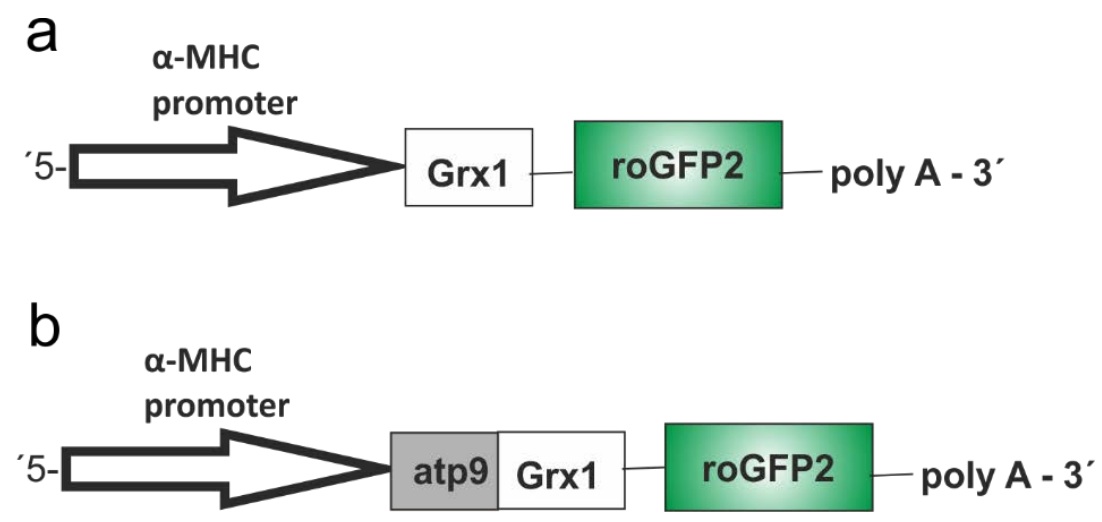

Figure 9: Schematic of the plasmids used for the generation of cyto Grx1-roGFP2 and mito Grx1-roGFP2 transgenic mouse models. (a) a-MHC promoter driven Grx1-roGFP2 biosensor is located in the cytoplasm of the cardiomyocytes and (b) is targeted to the mitochondrial matrix with the help of the Neurospora crassa ATP synthase protein 9 (atp9) signal sequence.

\subsubsection{Confirmation of the Grx1-roGFP transgene by genotyping}

To confirm the presence of the transgene in the respective mouse lines, genotyping was performed with the gDNA obtained from tail biopsies of offsprings by standard PCR. In the case of the cyto Grx1-roGFP2 transgene, the primers were designed in such a way that the forward primer binds to a sequence in the $\alpha-\mathrm{MHC}$ promoter and the reverse primer binds in the coding region of Grx1-roGFP. In case of the mito Grx-roGFP2 transgene, the forward primer binds to a sequence of the $\alpha-\mathrm{MHC}$ promoter and the reverse primer binds in the coding region of the Grx1.

Since the transgenesis was non-directed, the exact insertion of the transgenic DNA sequence in the genome of the mouse models is not known. This lack of information precluded designing primers which would cover for example the plasmid insertion start or end. Therefore the genotyping PCR primers used in this study could only cover the transgenic DNA. In line, the genotyping PCR will result in a band in PCRs performed with tail biopsies from transgenic mice and no band in the wild type mice. PCR reactions performed with tail biopsies are prone to failure based on the relatively non-purity of the isolated gDNA. Therefore a lack of a band in the PCR could either mean that the biopsy was taken from a real wild type animal as discussed above or that the PCR reaction failed. To distinguish these two possibilities, I included a PCR quality control in the genotyping PCR. The quality control was performed by including a second primer pair in the same PCR reaction. The second primer pair was designed to bind to the wild type background, not affected by the transgenic insertion. I selected for this the Phd2 gene, 
which indeed proved to be free of transgenic insertion. Having two primer sets in the genotyping PCR allows to distinguish wild type and transgenic littermates by the appearance of either one band (200 bp band) or two bands (200 bp and 500 bp for cyto or 700 bp for mito), respectively. A PCR failure would result in no bands on the agarose gel. From a pool of offsprings genotyped, 5 different mice's genotyping data sets as an example from each cyto Grx1-roGFP2 and mito Grx1-roGFP2 mouse lines are shown in Fig.10a and Fig. 10b.
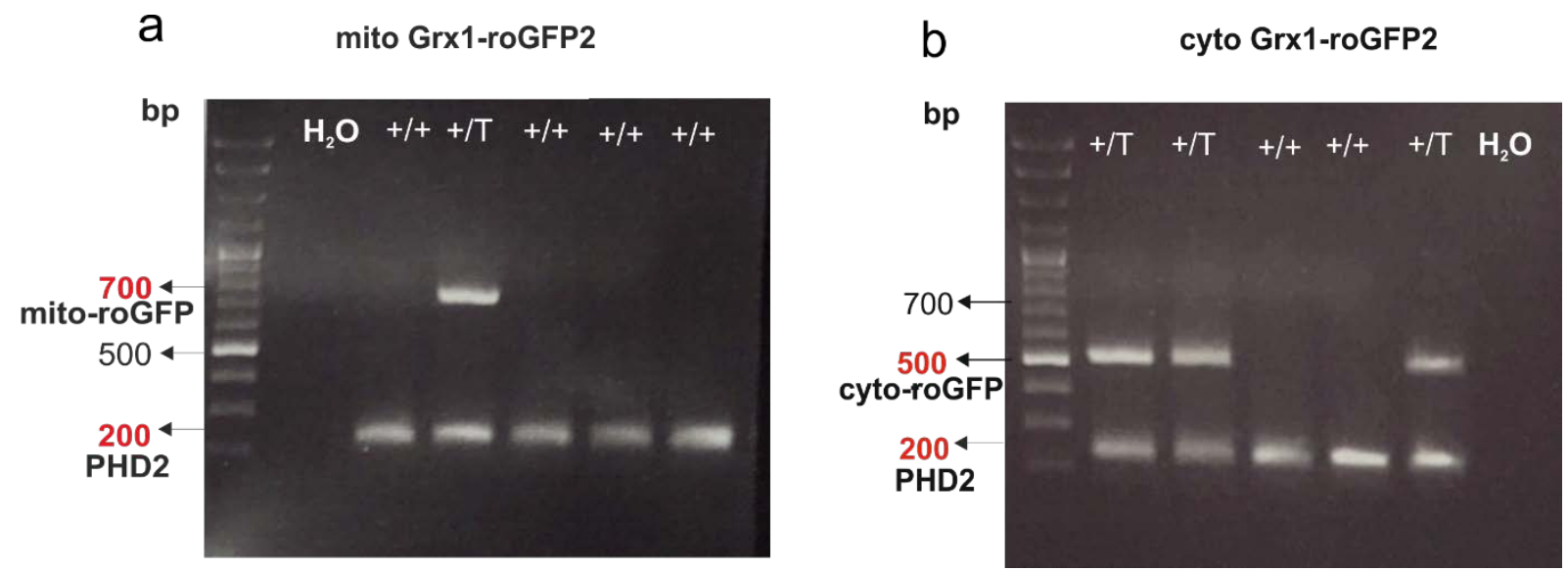

Figure 10: Genotyping results confirming the presence of the transgene in the mito Grx1-roGFP2 and cyto Grx1-roGFP2 mice. (a) A 2\% agarose gel containing the PCR products showing the presence of the wild type control gene Phd2 (200 bp) and the transgene roGFP (700 bp) in 5 different mice from the mito Grx1-roGFP2 breeding. (b) A $2 \%$ agarose gel containing the PCR products showing the presence of the wild type control gene Phd2 (200 bp) and the transgene roGFP (500 bp) in 5 different mice from the cyto Grx1-roGFP2 breeding. Each agarose gel includes a molecular size marker and a PCR water $\left(\mathrm{H}_{2} \mathrm{O}\right)$ control reaction. $+/ T$ (heterozygous transgenic genotype), +/+ (wild type genotype).

\subsubsection{Typical response of isolated Grx1-roGFP2 cardiomyocytes to $\mathrm{H}_{2} \mathrm{O}_{2}$ and DTT}

For confirming the functionality of the Grx1-roGFP2 biosensor in the transgenic mouse models, control experiments with isolated cardiomyocytes were performed. Cardiomyocytes were isolated from the transgenic mito and cyto Grx1-roGFP2 mouse lines by Langendorff's perfusion system. Live time imaging was performed on isolated cardiomyocytes using inverted epifluorescence microscopy. The roGFP probe was excited at 405 and $488 \mathrm{~nm}$ and the emitted light was detected by a CCD camera at $510 \mathrm{~nm}$. Prior to addition of any oxidizing or reducing agent for each coverslip containing cardiomyocytes, a baseline was recorded for approximately $50 \mathrm{~s}$. Subsequently, $\mathrm{H}_{2} \mathrm{O}_{2}$ as an oxidizing agent and DTT as a reducing agent were added on independent coverslips. The changes in the fluorescence intensity were recorded until a plateau phase was reached. The normalized ratio of 405/488 nm excitation wavelengths was plotted in correspondence to changes in the absolute fluorescence intensities of 405 and $488 \mathrm{~nm}$ after addition of the oxidizing or reducing agent. Fig. 11 indicates 
the normalized ratio of $405 / 488 \mathrm{~nm}$ obtained from a combined set of data on addition of 100 $\mu \mathrm{M} \mathrm{H}_{2} \mathrm{O}_{2}$ or $2 \mathrm{mM}$ DTT to the cardiomyocytes isolated from a transgenic mito and a cyto Grx1roGFP2 mouse. There is an increase observed in the fluorescence intensity at $405 \mathrm{~nm}$ and a decrease at $488 \mathrm{~nm}$ on addition of $\mathrm{H}_{2} \mathrm{O}_{2}$, whereas an opposite change is observed in the fluorescence intensities after addition of DTT.
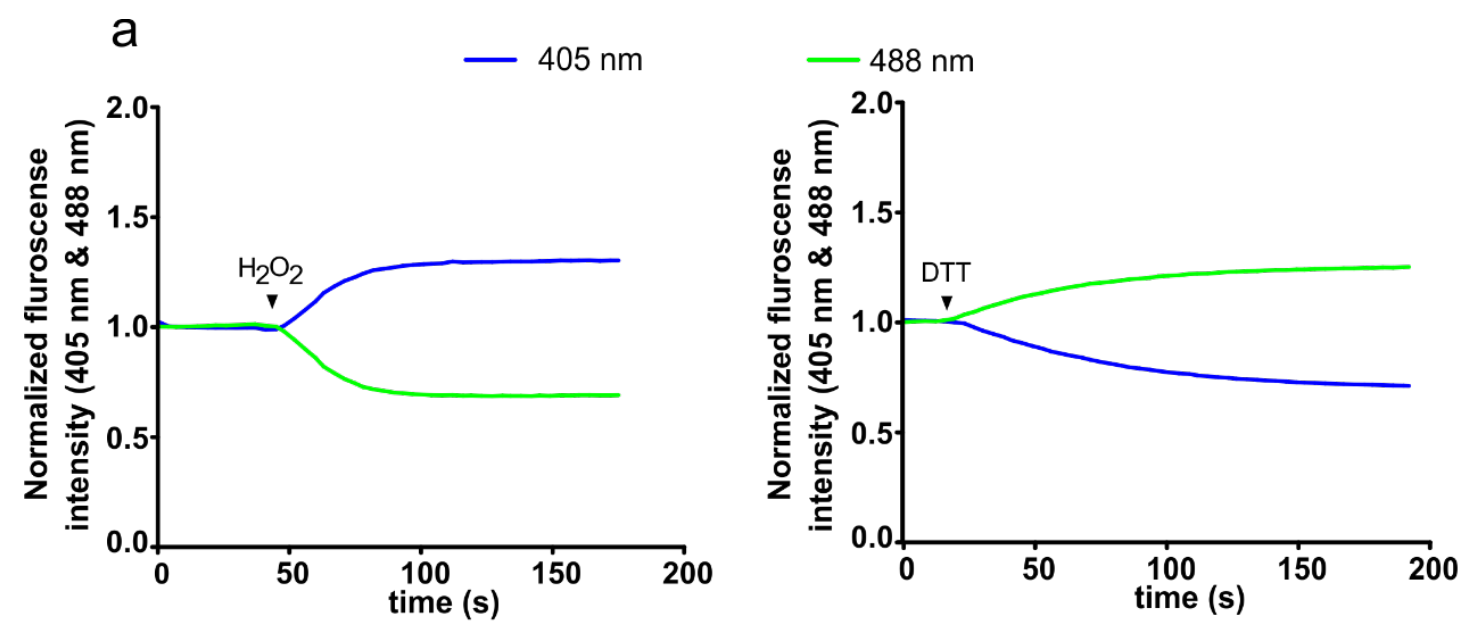

b
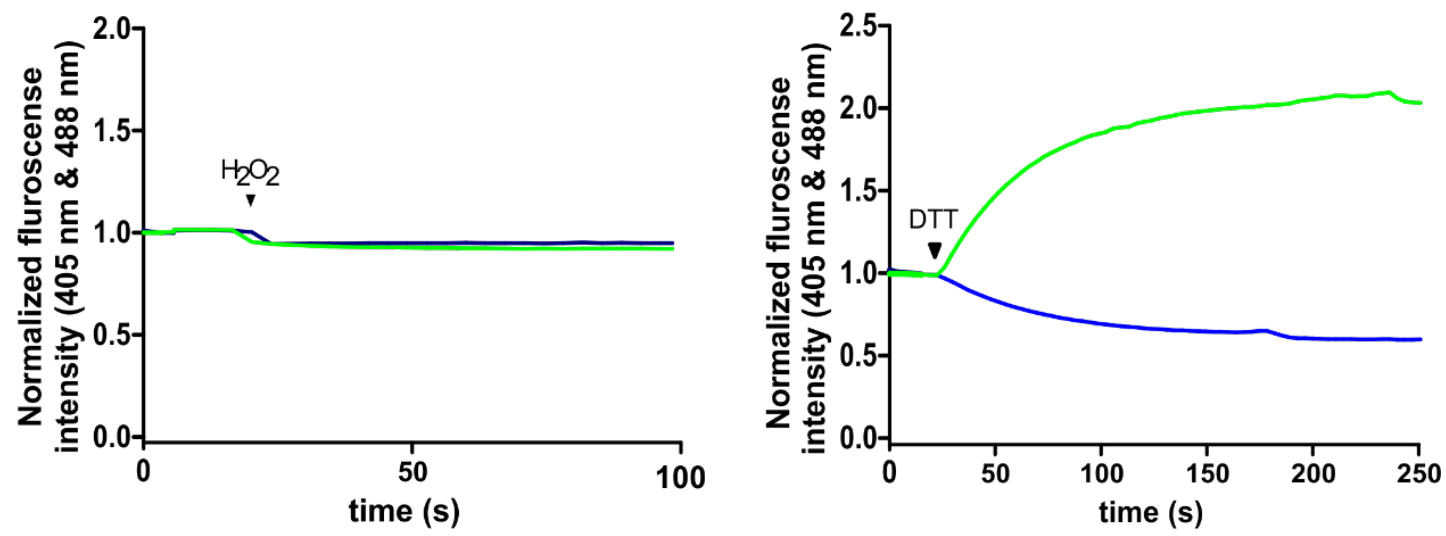

Figure 11: Typical response of Grx1-roGFP cardiomyocytes to $\mathrm{H}_{2} \mathrm{O}_{2}$ and DTT. Cardiomyocytes isolated from (a) a mito Grx1-roGFP2 mouse and (b) a cyto Grx1-roGFP2 were measured on an inverted epifluorescence microscope for the changes in fluorescence intensities after addition of an oxidizing $\left(\mathrm{H}_{2} \mathrm{O}_{2}\right)$ and a reducing (DTT) compound. Normalized ratio of fluorescence excitations at $405 / 488 \mathrm{~nm}$ was calculated after addition of $100 \mu \mathrm{M} \mathrm{H} \mathrm{H}_{2} \mathrm{O}_{2}$ and $2 \mathrm{mM}$ DTT respectively to the isolated cardiomyocytes.

\subsubsection{EGSH of isolated cardiomyocytes from Grx1-roGFP2 mice}

Offsprings from in total two independent mouse lines for transgenic mice expressing the Grx1roGFP2 in the cytosol (cyto1 and cyto2) and the mitochondrial matrix (mito1 and mito2) were included in the following experiments. The inclusion of two independent mouse lines is thought to prevent analyzing founder-dependent rather than general effects. Cardiomyocytes were isolated from each of the two independent founder lines of cyto Grx1-roGFP2 (cyto1 and cyto 2) and mito Grx1-roGFP2 (mito1 and mito2) by Langendorff's perfusion system. These 
cardiomyocytes were used for measuring $E_{\mathrm{GSH}}$ with the help of a set of two different oxidizingreducing agents namely $100 \mu \mathrm{M}$ diamide-2 mM DTT and $100 \mu \mathrm{M} \mathrm{H}_{2} \mathrm{O}_{2}-2$ mM DTT. $\mathrm{H}_{2} \mathrm{O}_{2}$ oxidizes the roGFP2 via the enzyme Grx1 whereas, diamide oxides the roGFP2 directly bypassing the enzyme Grx1. This makes it possible to use diamide as a control to confirm that the roGFP2 is fully oxidized. $E_{\mathrm{GSH}}$ were calculated by Nernst equation utilizing the changes in the fluorescence intensities of 405 and $488 \mathrm{~nm}$ roGFP excitation wavelengths obtained after addition of the oxidizing-reducing agent (Table 18). Interestingly, the Grx1-roGFP2 biosensor gave a lower response to DTT when placed in the mitochondrial matrix compared to being placed in the cytosol. This indicates a lower capacity of the mitochondrial matrix to reduce any further suggesting that the mitochondrial matrix of the cardiomyocytes is already highly reduced to as low as $-278.9 \pm 0.4 \mathrm{mV}$ (mito1) and $-277 \pm 0.4 \mathrm{mV}$ (mito2). The opposite phenomenon is observed in case of the cyto mice giving a higher response to DTT, suggesting it to be in an oxidized form at baseline. In line, the cytoplasm of the cardiomyocyte was found to have an $E_{\mathrm{GSH}}$ as high as $-257.2 \pm 0.7 \mathrm{mV}$ (cyto1) and $-255.8 \pm 0.6 \mathrm{mV}$ (cyto2). Taken together, the data suggest that cardiomyocytes exhibit a specific redox compartmentalization in the cytoplasm versus the mitochondrial matrix.

\begin{tabular}{c|c|c|c|c}
\hline \multicolumn{5}{|c}{ Mouse line } \\
\hline EGSH & cyto1 & cyto2 & mito1 & mito2 \\
& & & & \\
Diamide - DTT & $\begin{array}{c}-257.5 \pm 0.7 \mathrm{mV} \\
(102)\end{array}$ & $-255.8 \pm 0.6 \mathrm{mV}$ & $-278.9 \pm 0.4 \mathrm{mV}$ & $-277.1 \pm 0.4 \mathrm{mV}$ \\
& & $(154)$ & $(108)$ & $(97)$ \\
$\mathrm{H}_{2} \mathrm{O}_{2}-$ DTT & $-254.8 \pm 0.8 \mathrm{mV}$ & $-256.3 \pm 0.7 \mathrm{mV}$ & $-278.0 \pm 0.4 \mathrm{mV}$ & $-275.4 \pm 0.4 \mathrm{mV}$ \\
& $(112)$ & $(154)$ & $(104)$ & $(98)$ \\
& & & & \\
\hline
\end{tabular}

Table 18 : Cardiomyocytes exhibit a specific redox compartmentalization in $E_{\mathrm{GSH}}$ of the mitochondrial matrix versus the cytoplasm. Isolated cardiomyocytes from the indicated mouse lines (cyto1, cyto2, mito1 and mito2) were measured for glutathione redox potential $\left(E_{\mathrm{GSH}}\right)$ with $100 \mu \mathrm{M}$ diamide-2 $\mathrm{mM}$ DTT and $100 \mu \mathrm{M} \mathrm{H}_{2} \mathrm{O}_{2}-2 \mathrm{mM}$ DTT as an oxidizing and reducing agent. The numbers in the brackets represent the numbers of cardiomyocytes measured from each line. $E_{\mathrm{GSH}}$ were calculated as described in Materials and Methods and are shown as mean +/- SEM.

\subsubsection{Echocardiography of ageing mito Grx1-roGFP2 and cyto Grx1-roGFP2 mice as compared to young mito Grx1-roGFP2 and cyto Grx1-roGFP2 mice}

Having established that the $E_{\mathrm{GSH}}$ is compartmentalized in cardiomyocytes, I was interested to exploit the Grx1-roGFP2 mouse models to study the effects of ageing on the $E_{G S H}$. For this purpose, 5 and 3 mice from the cyto Grx1-roGFP2 and mito Grx1-roGFP2 mouse lines 
respectively were included in the analysis. Echocardiography was done on these mice from the age of 23 weeks on to as old as 70 and 80 weeks. EF and FAS were recorded as markers for cardiac function. It was observed that the FAS and the EF of mice from both the mouse lines, i.e. cyto and mito, were significantly decreased at the age of 80 weeks (Fig. 12 a and b). These data demonstrate an age-dependent drop of heart function indicating that the time points chosen are suitable to analyze age-dependent effects.

a

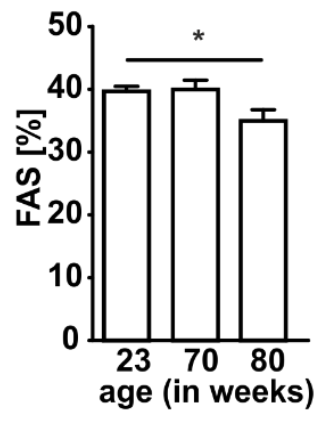

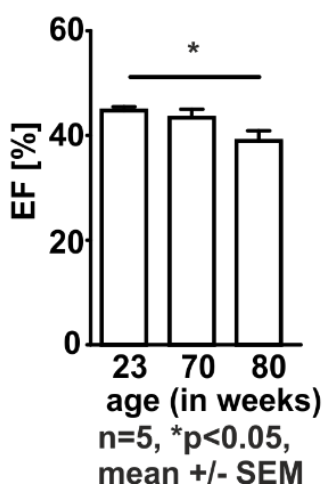

b

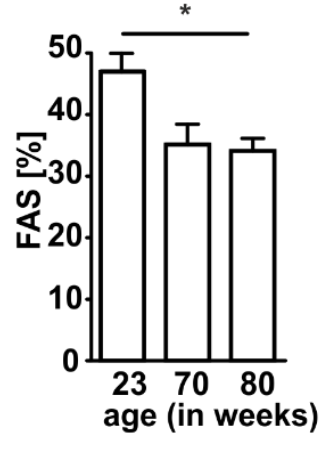

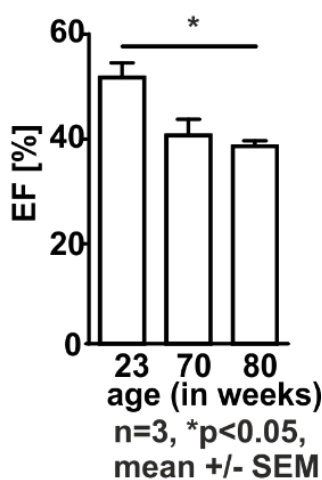

Figure 12: Grx1-roGFP2 aged mice show significantly poorer heart function than Grx1roGFP2 young mice. Fractional Area Shortening (FAS) and Ejection Fraction (EF) were recorded by echocardiography from the mice of age 23 weeks to 80 weeks in the (a) transgenic cyto Grx1-roGFP2 mouse line ( $n=5$ mice) and the (b) transgenic mito Grx1-roGFP2 mouse line $\left(n=3\right.$ mice). ${ }^{*} p<0.05$.

\subsubsection{EGSH in isolated cardiomyocytes from mito Grx1-roGFP2 and cyto Grx1- roGFP2 aged mice}

Once it was verified that the heart function in the cyto Grx1-roGFP2 and mito Grx1-roGFP2 mice drops with ageing, the subsequent step was to measure $E_{G S H}$ from these mice. To this end, $E_{\mathrm{GSH}}$ of the cyto Grx1-roGFP2 and mito Grx1-roGFP2 mice from two age groups, i.e. 20 and 80 weeks old, were analyzed. $100 \mu \mathrm{M} \mathrm{H}_{2} \mathrm{O}_{2}-2 \mathrm{mM}$ DTT was used as an oxidizing and reducing agent for the analysis. It was noticed that there was no difference in the $E_{\mathrm{GSH}}$ of cardiomyocytes isolated from both age groups of mito Grx1-roGFP2 mice being as reduced as $-276.1 \pm 1.0 \mathrm{mV}$ and $-276.5 \pm 0.8 \mathrm{mV}$ in case of 20 weeks and 80 weeks old mice, respectively. In case of cardiomyocytes isolated from 80 weeks old cyto Grx1-roGFP2 the $E_{\mathrm{GSH}}(-278.1 \pm$ $0.6 \mathrm{mV}$ ), however, was significantly more reduced than in cardiomyocytes from 20 weeks old mice $\left(-262.3 \pm 1.0 \mathrm{mV}\right.$ ) (Table 19). These data indicate that ageing affects the $E_{\mathrm{GSH}}$ in the cytosol and mitochondrial matrix differentially. 


\begin{tabular}{|c|c|c|c|c|}
\hline \multicolumn{5}{|c|}{ compartment } \\
\hline$E_{G S H}$ & $\begin{array}{c}\text { cyto } \\
\text { age, } 20 \text { weeks }\end{array}$ & $\begin{array}{c}\text { cyto } \\
\text { age, }>80 \text { weeks }\end{array}$ & $\begin{array}{c}\text { mito } \\
\text { age, } 20 \text { weeks }\end{array}$ & $\begin{array}{c}\text { mito } \\
\text { age, }>80 \text { weeks }\end{array}$ \\
\hline $\mathrm{H}_{2} \mathrm{O}_{2}$ - DTT & $\begin{array}{c}-262.3 \pm 1.0 \mathrm{mV}^{\mathrm{a}} \\
(48)\end{array}$ & $\begin{array}{c}-278.1 \pm 0.6 \mathrm{mV}^{\mathrm{b}} \\
(189)\end{array}$ & $\begin{array}{c}-276.1 \pm 1.0 \mathrm{mV} \\
(43)\end{array}$ & $\begin{array}{c}-276.5 \pm 0.8 \mathrm{mV} \\
(88)\end{array}$ \\
\hline
\end{tabular}

a $p<0.05$, cyto, age 20 weeks compared to mito, age 20 weeks

${ }^{\mathrm{b}} \mathrm{p}<0.05$, cyto, age 20 weeks compared to cyto, age $>80$ weeks

Table 19: Redox compartmentalization between mitochondrial matrix and cytoplasm of the cardiomyocyte tends to disappear with ageing. Both the age groups of 20 weeks and $>80$ weeks old mice from the cyto Grx1-roGFP2 mouse line (cyto) and the mito Grx1-roGFP2 mouse line (mito) were used for measuring $E_{\mathrm{GSH}}$ from isolated cardiomyocytes. The numbers in the brackets represent the numbers of cardiomyocytes measured from each line. $E_{\mathrm{GSH}}$ was calculated as mean $+/$ - SEM from all measured cardiomyocytes.

\subsection{Oxygen affects the $E_{G S H}$ in isolated mitochondria}

\subsubsection{Redox histology performed with heart sections from mito Grx1roGFP2 mice}

Determining the $E_{\mathrm{GSH}}$ in isolated cells is the most common method described in the literature when genetically biosensors are used. This method, however, precludes analyzing the cells when placed in their natural tissue context. Fujikawa et al., 2016 have recently published a redox histology method that allows determining the $E_{\mathrm{GSH}}$ in cryosections. I applied this method to cardiac cryosections obtained from the mito Grx1-roGFP2 mice. The cardiac tissue was cryosectioned into $14 \mu \mathrm{m}$ thin sections inside a cryostat machine which was precooled to -20 ${ }^{\circ} \mathrm{C}$. Henceforth, all the steps were done on ice. Four independent sections were taken onto the respective glass slides, which were incubated with $1 \mathrm{mM}$ diamide, $20 \mathrm{mM}$ DTT, $50 \mathrm{mM}$ NEM and PBS respectively for 10 minutes. NEM is a chemical which blocks thiol groups and hence freezes the redox status of molecules. Following the mounting of the sections, the cryosections were visualized using a confocal microscope. Fig. 13 shows a typical confocal image of the cryosections. The roGFP2 probe was excited at 405 and $488 \mathrm{~nm}$ and the emitted light was obtained at $510 \mathrm{~nm}$. The fluorescence intensities at the wavelengths of $405 \mathrm{~nm}$ and $488 \mathrm{~nm}$ were recorded in the diamide, DTT, NEM and non-treated (PBS) sections respectively. The upper panel in Fig. 13 shows the fluorescence intensities at $405 \mathrm{~nm}$ and $488 \mathrm{~nm}$ wavelengths of each reagent treatment in a transgenic versus wild type cardiac sections. It also represents the nuclear stain obtained from To-Pro-3 dye in a transgenic versus wild type tissue sections. The lower panel of Fig.13 represents a merged image of the fluorescence intensity ratio of 405 $\mathrm{nm} / 488 \mathrm{~nm}$ in all the respective reagent treatments. A dotted intracellular pattern was observed in both the excitation wavelengths. Since the biosensor is expressed in the mitochondrial 
matrix of cardiomyocytes the dots are representing the cardiac mitochondria. This was also supported by the observation that there was no signal obtained from the cryosections of the wild type control mouse. Red stained nuclei were visible in the cryosections obtained from the mito Grx1-roGFP2 as well as the wild type control mice.
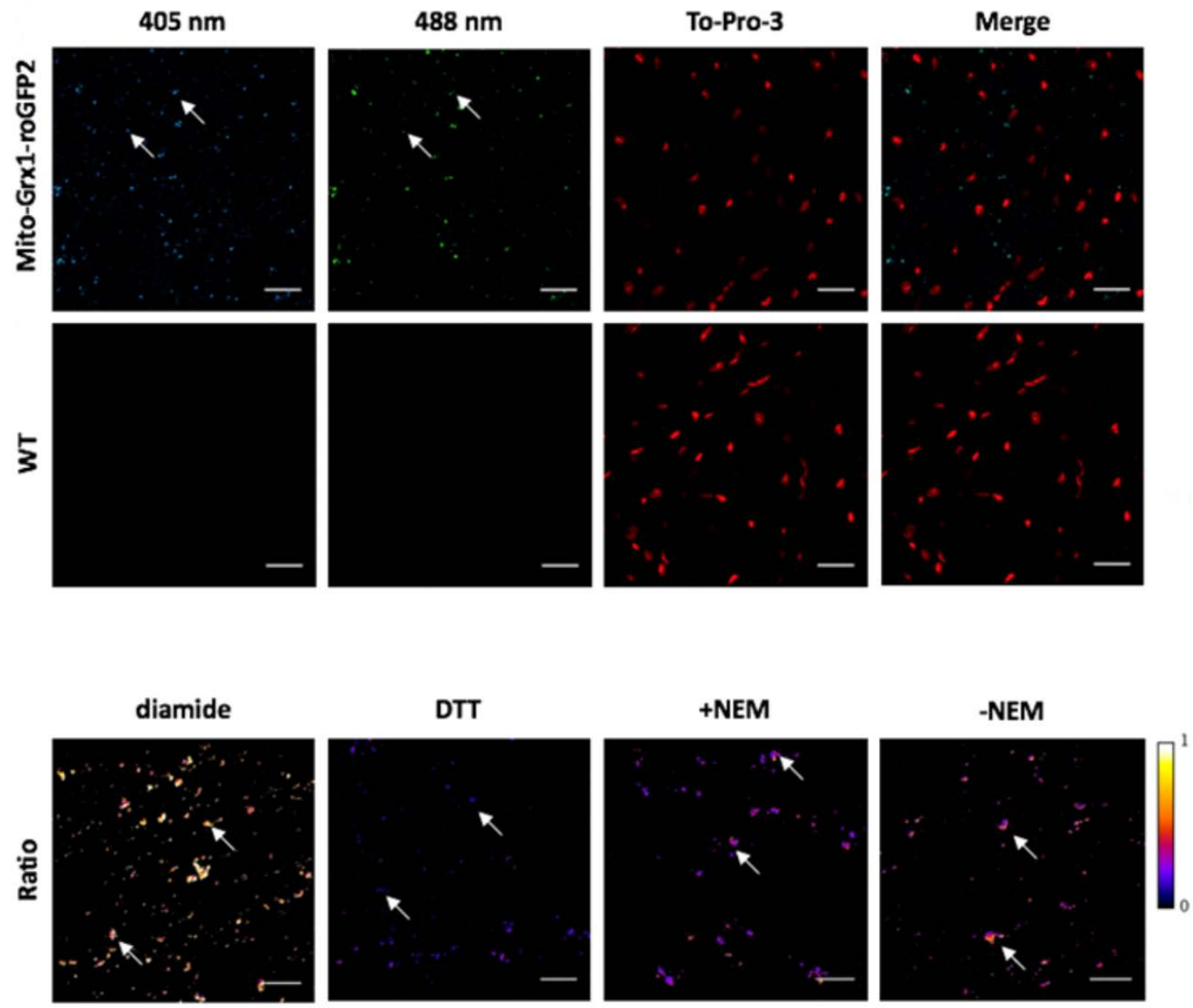

Figure 13: Redox histology performed with cryosections obtained from a wild type and a mito Grx1-roGFP2 mouse. Upper panel: Cryosections of the left ventricle obtained from a wild type (WT) and a mito Grx1-roGFP2 mouse were analyzed at the indicated wavelengths and for the nucleus staining To-Pro-3. Lower panel: Cryosections of the left ventricles were analyzed. Spots indicated as examples by the arrows represent the Grx1-roGFP expressed in the mitochondrial matrix. DTT and diamide were used to achieve full reduction or oxidation of the Grx1-roGFP, respectively. The values obtained from the fully reduced and oxidized sections were used for analyzing the cryosections, in which the redox status was either fixed with NEM or left untreated (-NEM). The heat map was calculated based on the ratio (excitations at $405 / 488 \mathrm{~nm}$ ) of the fully oxidized state after treatment with diamide $(=1)$ and the fully reduced state after treatment with DTT $(=0)$.

\subsubsection{Comparison of the mtOxD in cardiac sections, isolated cardiomyocytes and isolated cardiac mitochondria}

Having established the redox histology protocol, I compared the OxD obtained from analyzing isolated cardiomyocytes, heart cryosections and isolated cardiac mitochondria. The OxD 
reflects the \% oxidation of the total GSH pool, whereas the $E_{G S H}$ is a defined value. For the comparison of the isolated cardiomyocytes, cryosections and isolated mitochondria, the OxD rather than the $E_{G S H}$ was determined since it is unclear, if the absolute values obtained from the different methods applied are indeed comparable. Determining the OxD at tissue level relies on procedures to prevent oxidation of the redox biosensor during specimen dissection and fixation as described above. I likewise confirmed this when analyzing the mitochondrial mtOxD in cryosections of mtGrx1-roGFP2 transgenic mice (Fig. 14).

Incubation of the heart sections with NEM is protecting thiol groups preventing oxidation of the biosensor. Interestingly, incubation of isolated cardiomyocytes from mito Grx1-roGFP transgenic hearts with or without NEM did not alter the mtOxD indicating that the physiological intracellular milieu is sufficient for preserving the more reduced state of the mitochondrial matrix. Next I analyzed the mtOxD in isolated cardiac mitochondria from the mito Grx1-roGFP2 mice. The mtOxD of isolated mitochondria revealed a higher degree of oxidation compared to the isolated cardiomyocytes and was similar to the mtOxD of non NEM-treated cryosections (around 90\%). Similar to the histology sections, the shift of the mtOxD could be prevented by addition of NEM to all steps of the mitochondria isolation procedure. This finding suggests that when mitochondria are isolated from its normal physiological environment, the procedure per se has an impact on the OxD.

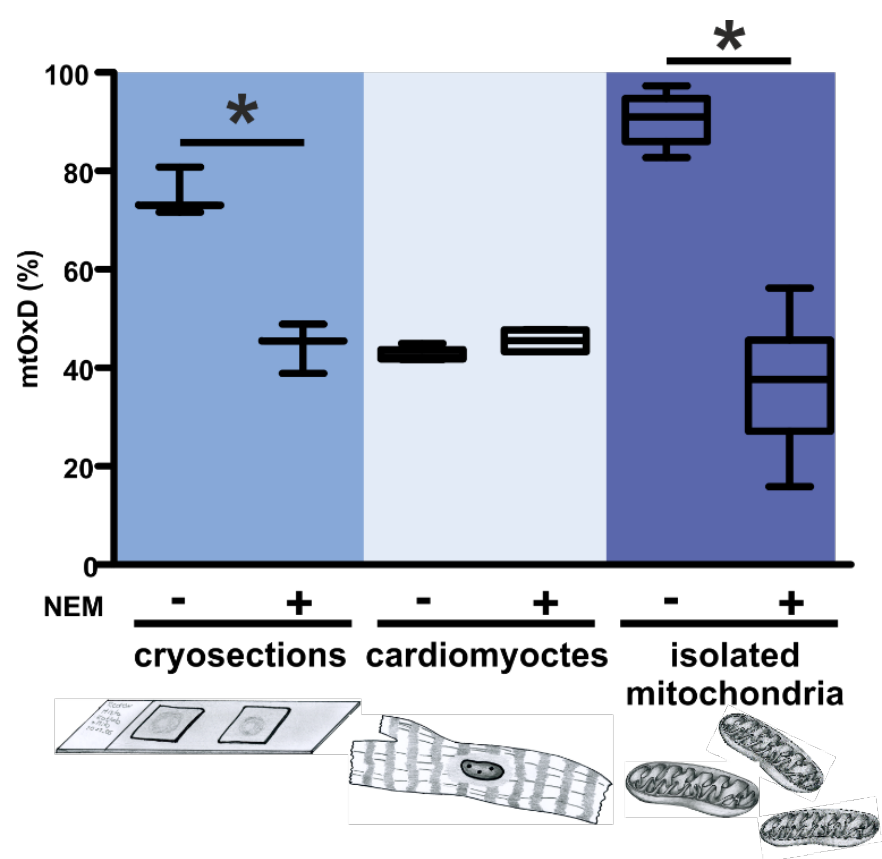

Figure 14: The glutathione pool of the cardiac mitochondrial matrix is oxidized during isolation of mitochondria. Mitochondrial oxidation differences (mtOxD (\%)) of cardiac cryosections ( $n=3$ mice), isolated cardiomyocytes ( $n=4$ mice) and isolated mitochondria $(n=9$ mice) from the transgenic mito Grx1-roGFP2 mouse line were measured by using $100 \mu \mathrm{M}$ diamide-2 mM DTT as oxidizing-reducing agents. Cryosectioning, isolation of cardiomyocytes and isolation of mitochondria were done in presence or absence of $\mathrm{N}$-ethylamide (NEM). mtOxD (\%) was calculated as mean $+/$ - SEM. * $p<0.05$. 


\subsubsection{EGSH and the mtOxD of cardiac mitochondria isolated from mito Grx1- roGFP2 mice in normoxia versus hypoxia}

The data acquired so far, strongly suggest that the physiological environment of cardiomyocytes is important to maintain the mitochondrial $E_{\mathrm{GSH}}$. To this end I was further interested in revealing that limiting factor which is able to maintain the redox status in the physiological environment of cardiomyocytes. Physiological oxygen concentration was speculated to be one of the players in serving to maintain the reduced mitochondrial $E_{\mathrm{GSH}}$. Whereas the $\mathrm{pO}_{2}$ that mitochondria are exposed to within the tissue and cellular context is thought to be $<1 \mathrm{mmHg}$, isolated mitochondria are exposed to a $\mathrm{pO}_{2}$ of $158 \mathrm{mmHg}$ at sea level. To mimic the low in situ oxygen conditions, cardiac mitochondria from transgenic mito Grx1roGFP2 mice were isolated in different oxygen concentrations ranging for $20.9 \% \mathrm{O}_{2}$ to $10 \%$, $3 \%$ and as low as $0.1 \% \mathrm{O}_{2}$. As indicated in Fig. 15, normoxic $\left(20.9 \% \mathrm{O}_{2}\right)$ isolation of mitochondria was done at room air whereas the hypoxic $\left(10 \%, 3 \%\right.$ and $\left.0.1 \% \mathrm{O}_{2}\right)$ isolation was done in an invivo2 400 hypoxia workstation. Hypoxic isolated mitochondria were measured for mtOxD and $E_{\mathrm{GSH}}$ in the Clariostar microplate reader maintaining the corresponding oxygen concentrations or including an acute hypoxia or reoxygenation step. $100 \mu \mathrm{M}$ diamide-2 mM DTT was used as an oxidizing and reducing agent respectively. mtOxD and $E_{G S H}$ were calculated by Nernst equation from the changes in the fluorescence intensities after addition of the oxidizing and reducing agent.

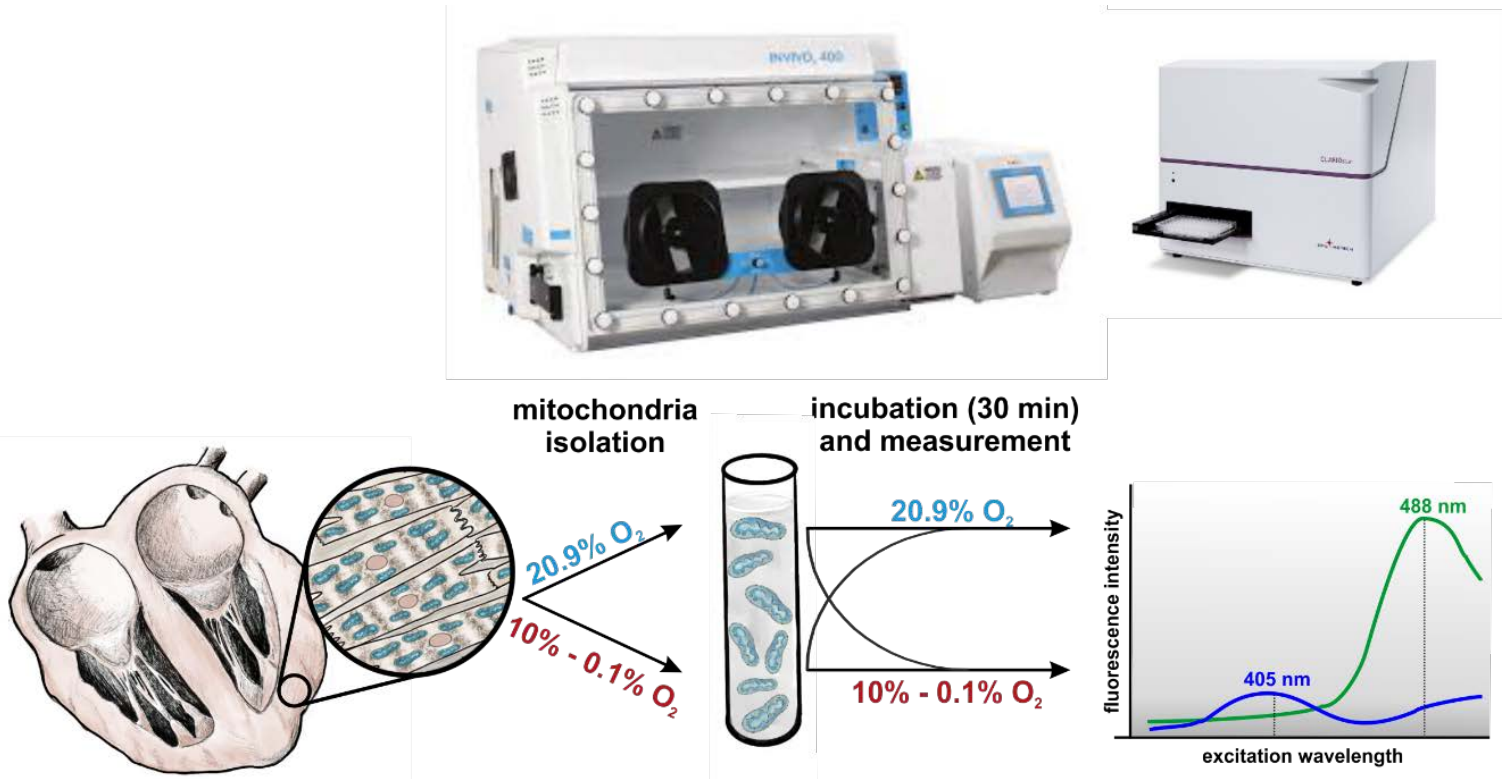

Figure 15: Schematic of the experimental setup for isolation of cardiac mitochondria from the mito Grx1-roGFP2 mouse line in normoxia versus hypoxia. Step-wise procedure to measure OXD and $E_{\mathrm{GSH}}$ of mitochondria isolated from the transgenic mito Grx1-roGFP2 mouse hearts. 
Mitochondria isolated in $20.9 \% \mathrm{O}_{2}$ showed a significant response to DTT and almost no response to diamide, indicating a highly oxidized $E_{\mathrm{GSH}}$ (Fig. 16a and b). When the oxygen concentration during isolating the mitochondria was maintained as low as $0.1 \% \mathrm{O}_{2}$ and $3 \% \mathrm{O}_{2}$, the mitochondria showed a higher response to diamide suggesting its higher capacity to get oxidized as compared to mitochondria isolated in $20.9 \% \mathrm{O}_{2}$. Mitochondria isolated in $10 \% \mathrm{O}_{2}$ displayed an OxD and $E_{\mathrm{GSH}}$ very similar to normoxic-isolated mitochondria, indicating $10 \% \mathrm{O}_{2}$ concentration as a threshold oxygen concentration which is enough to almost completely oxidize the mitochondrial glutathione pool. Altogether these experiments indicate that the ambient oxygen significantly affects the $E_{\mathrm{GSH}}$ of isolated mitochondria, which is of interest since isolation of mitochondria at room air conditions is a common routine in cell and molecular biology protocols.
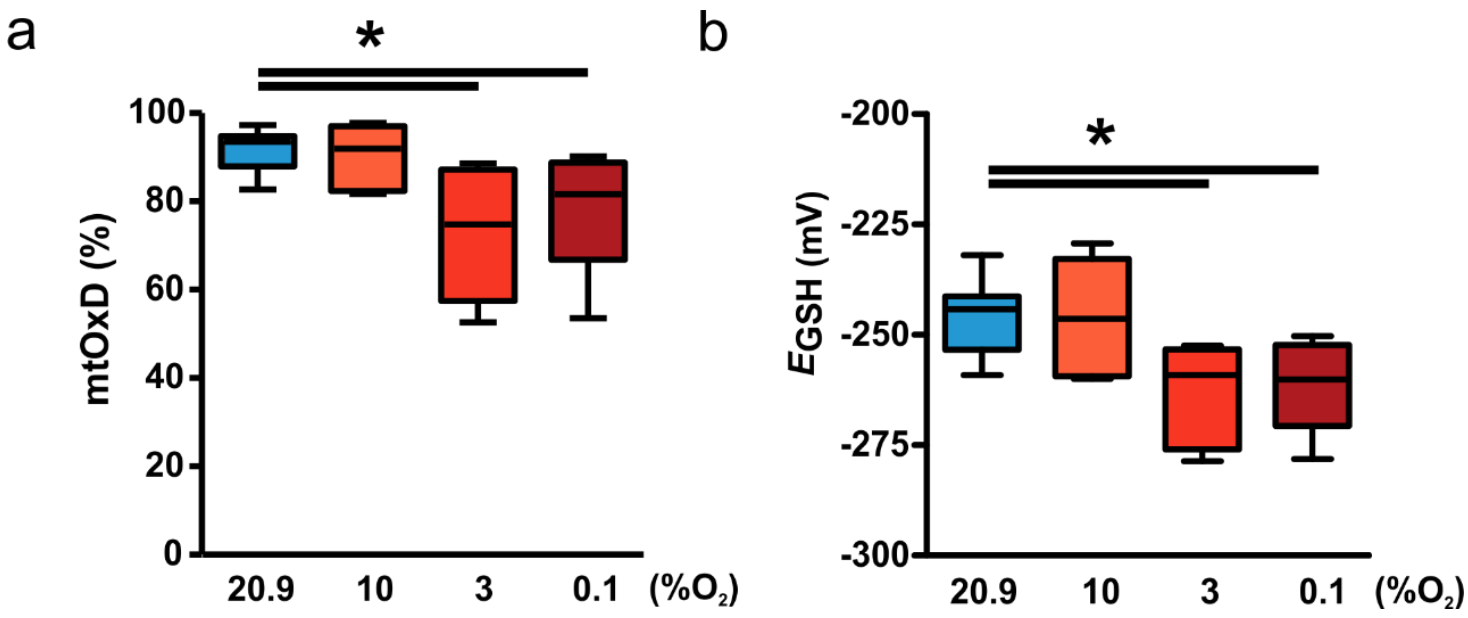

Figure 16: Isolation of mitochondria in hypoxia preserves the reduced $E_{\mathrm{GSH}}$ and $\mathrm{mtOxD}$ (\%) of the mitochondrial matrix. Mitochondria were isolated from cardiac tissue of transgenic mito Grx1-roGFP2 mice in $20.9 \% \mathrm{O}_{2}(\mathrm{n}=11$ mice) versus $10 \%$ ( $\mathrm{n}=6$ mice), $3 \%(\mathrm{n}=6$ mice) and $0.1 \% \mathrm{O}_{2}$ ( $\mathrm{n}=11$ mice). (a) Mitochondrial Oxidation Difference (mtOxD (\%)) and (b) glutathione redox potential $\left(E_{\mathrm{GSH}}\right)$ of these mitochondria were further measured at the respective oxygen concentration. mtOxD (\%) and $E_{\mathrm{GSH}}$ were calculated as mean $+/$ - SEM of individual experiments. * $p<0.05$.

\subsubsection{EGSH and the mtOxD of cardiac mitochondria isolated from mito Grx1- roGFP2 mice during reoxygenation and acute hypoxia}

The data so far suggest that mitochondria can maintain their redox status when isolated in hypoxia. To test, if this effect is reversible I performed acute reoxygenation and acute hypoxia experiments. In detail I analyzed the mtOxD and $E_{G S H}$ in hypoxic isolated mitochondria when incubated in a normoxic environment (reoxygenation) as well as the mtOxD and $E_{G S H}$ in normoxic isolated mitochondria when incubated in a hypoxic environment (acute hypoxia). Mitochondria were isolated in $20.9 \% \mathrm{O}_{2}$ and $0.1 \% \mathrm{O}_{2}$ from transgenic mito $\mathrm{Grx} 1$-roGFP2 mouse hearts. Mitochondria isolated in $0.1 \% \mathrm{O}_{2}$ were allowed to reoxygenate by incubating them in $20.9 \% \mathrm{O}_{2}\left(0.1 \% \mathrm{O}_{2} \longrightarrow 20.9 \% \mathrm{O}_{2}\right)$ for $30 \mathrm{~min}$. $E_{\mathrm{GSH}}$ of reoxygenated mitochondria were measured at the corresponding oxygen concentration by using $100 \mathrm{mM}$ diamide-2 mM DTT as 
an oxidizing and reducing agent, respectively. Similarly, mitochondria isolated in normoxia were exposed to acute hypoxia and therefore incubated in $0.1 \% \mathrm{O}_{2}\left(20.9 \% \mathrm{O}_{2} \longrightarrow 0.1 \% \mathrm{O}_{2}\right)$ for $30 \mathrm{~min}$. $E_{\mathrm{GSH}}$ of the mitochondria were measured at the corresponding oxygen concentration by using $100 \mathrm{mM}$ diamide-2 $\mathrm{mM}$ DTT as an oxidizing and reducing agent, respectively. Interestingly, it was observed that reoxygenated mitochondria get fully oxidized and have an $E_{\mathrm{GSH}}$ similar to mitochondria isolated at $20.9 \% \mathrm{O}_{2}$ (Fig. 17a). On the other hand, acute hypoxic incubation of mitochondria did not show any effect on $E_{G S H}$ (Fig. 17b). Mitochondria were completely oxidized like normoxic isolated mitochondria. This indicates that the changes induced at the atmospheric oxygen levels are the result of an irreversible process.

a

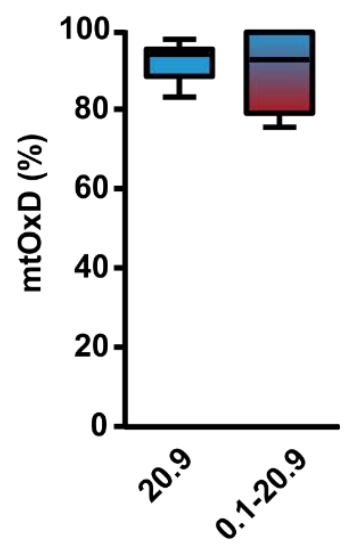

$\left(\% \mathrm{O}_{2}\right)$

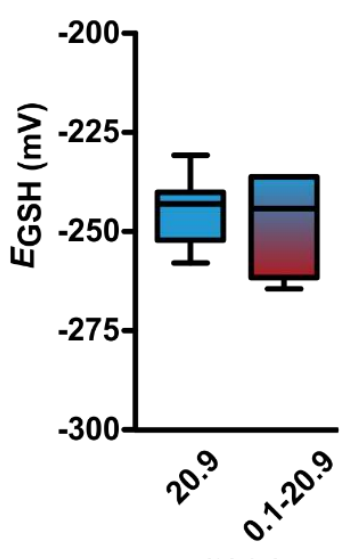

$\left(\% \mathrm{O}_{2}\right)$ b

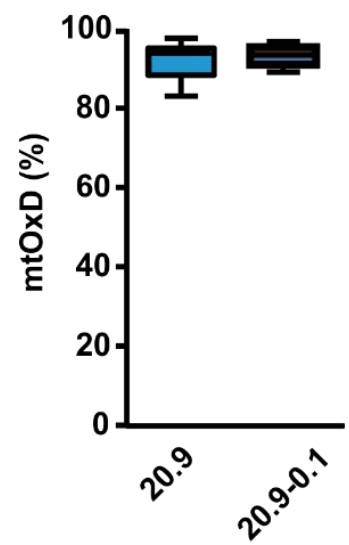

$\left(\% \mathrm{O}_{2}\right)$

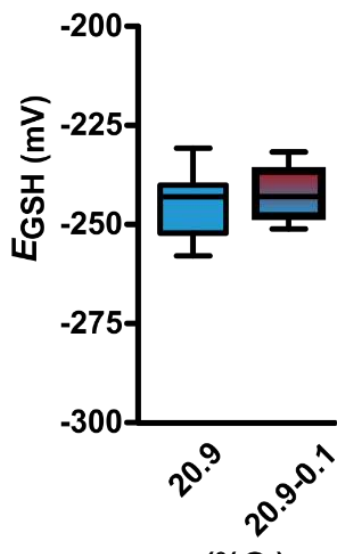

$\left(\% \mathrm{O}_{2}\right)$

Figure 17: Exposing isolated mitochondria to normoxia results in an irreversible oxidation of mitochondrial OxD and $E_{\mathrm{GSH}}$. Mitochondria isolated from cardiac tissue of mito Grx1-roGFP2 mice in $20.9 \% \mathrm{O}_{2}$ and $0.1 \% \mathrm{O}_{2}$ were incubated and analyzed for mitochondrial Oxidation Difference (mtOxD (\%)) (a) and glutathione redox potential $\left(E_{\mathrm{GSH}}\right)$ (b) at the respective oxygen concentrations $\left(20.9 \% \mathrm{O}_{2}\right.$ and $0.1 \% \mathrm{O}_{2}, \mathrm{n}=11$ mice $)$, reoxygenation $(0.1 \%$ $\mathrm{O}_{2} \longrightarrow 20.9 \% \mathrm{O}_{2}, \mathrm{n}=6$ mice) and acute hypoxia $\left(20.9 \% \mathrm{O}_{2} \longrightarrow 0.1 \% \mathrm{O}_{2}, \mathrm{n}=4\right.$ mice). mtOxD (\%) and $E_{G S H}$ were calculated as mean $+/$ - SEM of individual experiments. * $p<0.05$.

\subsubsection{Metabolically active mitochondria exhibit a reduction of the mtOxD and $E_{\mathrm{GSH}}$}

Succinate serves as a substrate to complex II in the mitochondrial electron transport chain. Stimulating mitochondria with succinate enhances the production of reducing equivalents like $\mathrm{NADPH}$ and thereby affecting the mitochondrial redox environment. In order to analyze the effect of succinate on the mtOxD and $E_{G S H}$, the following experiment was performed: Mitochondria were isolated in $20.9 \%$ and $0.1 \% \mathrm{O}_{2}$ from mito Grx1-roGFP2 hearts. A portion of $0.1 \% \mathrm{O}_{2}$ isolated mitochondria was kept in $20.9 \% \mathrm{O}_{2}$ for $30 \mathrm{~min}$ as a reoxygenation sample. Subsequently, mitochondria isolated in $0.1 \% \mathrm{O}_{2}, 20.9 \% \mathrm{O}_{2}$ and in the reoxygenation sample were incubated in presence or absence of $2.5 \mathrm{mM}$ succinate and $0.5 \mathrm{mM}$ ADP. These mitochondria were then measured for fluorescence intensities at 405 and $488 \mathrm{~nm}$ roGFP excitation wavelengths for $20 \mathrm{~min}$ at the corresponding oxygen concentrations. Following the 
baseline fluorescence intensities measurements, mtOxD (\%) (Fig. 18a) and $E_{G S H}$ (Fig. 18b) were determined by adding $100 \mu \mathrm{M}$ diamide-2 mM DTT as oxidizing and reducing agent. In these experiments, mitochondria isolated in $20.9 \% \mathrm{O}_{2}$ and energized with succinate/ADP got reduced to roughly $80 \%$ mtOxD as compared to mitochondria not stimulated with succinate/ADP. On the other hand, in case of mitochondria isolated and stimulated with succinate/ADP in $0.1 \% \mathrm{O}_{2}$, the mtOxD was significantly more reduced close to $40 \%$ as compared to mitochondria not stimulated with succinate/ADP. When the reoxygenated mitochondria were stimulated with succinate/ADP the mtOxD was oxidized back close to $70 \%$ as compared to hypoxic isolated mitochondria stimulated with succinate/ADP.

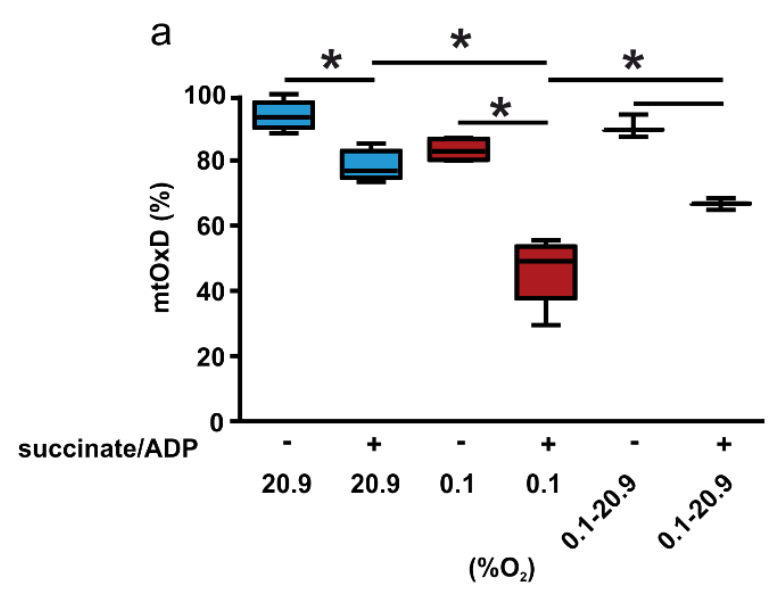

Figure 18: The mtOxD (\%) of mitochondria isolated and stimulated with succinate in hypoxia is significantly lower as compared to mitochondria isolated in normoxia. Mitochondria were isolated from hearts of transgenic mito Grx1-roGFP2 mice in $20.9 \% \mathrm{O}_{2}$ ( $n=4$ mice) and $0.1 \% \mathrm{O}_{2}$ ( $\mathrm{n}=4$ mice). A part of the $0.1 \% \mathrm{O}_{2}$ was incubated at $20.9 \% \mathrm{O}_{2}$ for 30 minutes $\left(0.1 \% \mathrm{O}_{2} \longrightarrow 20.9 \%\right)$. Subsequently, the mitochondria were stimulated without and with 2.5 $\mathrm{mM}$ succinate and $0.5 \mathrm{mM}$ ADP and measured for (a) mitochondrial Oxidation Difference $(\operatorname{mtOxD}(\%))$ and (b) $\mathrm{E}_{\mathrm{GSH}}$ at the respective oxygen concentration. OxD was calculated as mean $+/$ - SEM of individual experiments. * $p<0.05$.

\subsubsection{Superoxide anions levels in mitochondria isolated at $20.9 \%$ versus $0.1 \%$ $\mathrm{O}_{2}$}

In order to unravel the effect of low oxygen concentrations on the mitochondrial $E_{\mathrm{GSH}}$ in isolated mitochondria we analyzed ROS produced in the mitochondrial matrix. In line, mitochondrial $\mathrm{O}_{2}-$ levels were analyzed in mitochondria isolated and incubated with or without $2.5 \mathrm{mM}$ succinate and $0.5 \mathrm{mM}$ ADP at $20.9 \% \mathrm{O}_{2}$ versus $0.1 \% \mathrm{O}_{2}$ from wild type mouse hearts. The $\mathrm{O}_{2}$ levels were determined with the help of the MitoSOX fluorescence dye at the indicated oxygen concentrations. It was observed than mitochondria isolated in $0.1 \% \mathrm{O}_{2}$ produce significantly lower $\mathrm{O}_{2}-$ - levels than mitochondria isolated in $20.9 \% \mathrm{O}_{2}$ in both conditions, i.e. with or without succinate/ADP stimulation (Fig. 19a). As a control experiment, the MitoSox measurement was 
also performed at $0.1 \% \mathrm{O}_{2}$ with mitochondria, which were originally isolated in $20.9 \% \mathrm{O}_{2}$. This experiment was designed to rule out the possibility that the reduced $\mathrm{O}_{2} \cdot-$ levels in hypoxia are due to a technical artifact of the fluorescent dye measurement in hypoxia per se. The $\mathrm{O}_{2}$ superoxide anion levels measured in $20.9 \% \mathrm{O}_{2}$ isolated mitochondria in both oxygen concentrations, i.e. $20.9 \%$ and $0.1 \% \mathrm{O}_{2}$, however were similar excluding a technical artifact (Fig. 19b).

a

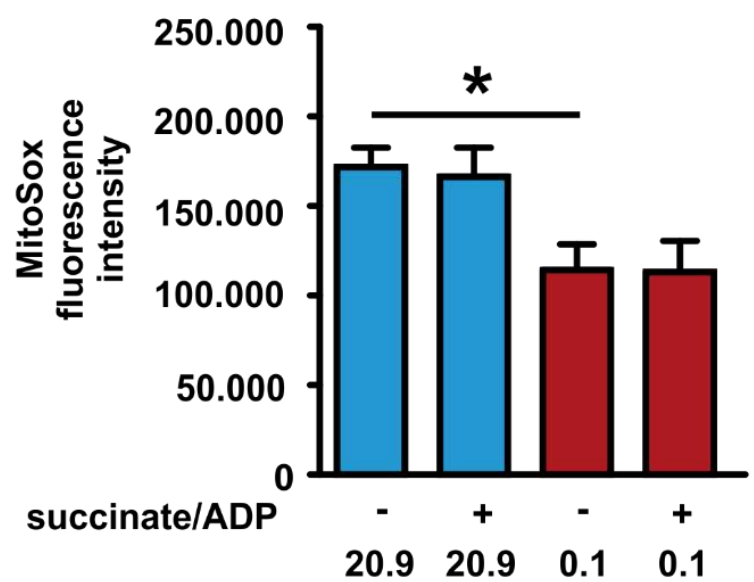

$\left(\% \mathrm{O}_{2}\right)$ b

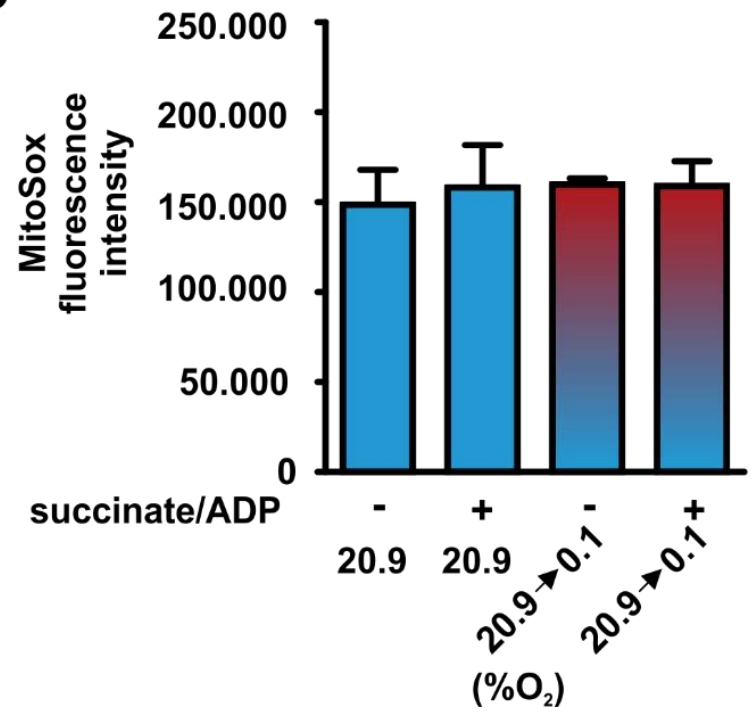

Figure 19: Superoxide anions levels are significantly lower in mitochondria isolated in hypoxia as compared to mitochondria isolated in normoxia. (a) Mitochondria isolated from wild type mouse hearts ( $\mathrm{n}=3$ mice) in $20.9 \% \mathrm{O}_{2}$ and $0.1 \% \mathrm{O}_{2}$ and incubated without or with 2.5 $\mathrm{mM}$ succinate and $0.5 \mathrm{mM}$ ADP as indicated, were measured at respective oxygen concentrations for $\mathrm{O}_{2}{ }^{-}$levels with the help of the MitoSOX fluorescence dye. (b) Mitochondria isolated in $20.9 \% \mathrm{O}_{2}$ incubated without or with $2.5 \mathrm{mM}$ succinate and $0.5 \mathrm{mM}$ ADP were measured at $0.1 \% \mathrm{O}_{2}\left(20.9 \% \mathrm{O}_{2} \longrightarrow 0.1 \% \mathrm{O}_{2}\right)$ for $\mathrm{O}_{2}{ }^{-}$levels as a control experiment. Fluorescence intensity was calculated as mean $+/$ - SEM of individual experiments. ${ }^{*} p<0.05$.

\subsubsection{Hydrogen peroxide levels in mitochondria isolated at $20.9 \%$ versus $0.1 \% \mathrm{O}_{2}$}

$\mathrm{O}_{2}^{--}$are immediately converted into $\mathrm{H}_{2} \mathrm{O}_{2}$ by superoxide dismutase in any biological system. Therefore, $\mathrm{H}_{2} \mathrm{O}_{2}$ levels were checked in mitochondria isolated and stimulated with $2.5 \mathrm{mM}$ succinate and $0.5 \mathrm{mM} \mathrm{ADP}$ at $20.9 \%$ and $0.1 \% \mathrm{O}_{2}$ from wild type mouse hearts. $\mathrm{H}_{2} \mathrm{O}_{2}$ levels were analyzed with the help of the Amplex Ultra red fluorescence dye. As shown in Fig. 20, there is a significantly higher amount of $\mathrm{H}_{2} \mathrm{O}_{2}$ produced by mitochondria isolated and stimulated with succinate/ADP in $20.9 \% \mathrm{O}_{2}$ as compared to mitochondria without succinate and ADP stimulation. In hypoxic isolated mitochondria, there was no increase observed in $\mathrm{H}_{2} \mathrm{O}_{2}$ production even after stimulation with succinate/ADP. These measurements hint towards a very tight electron flow going on in the mitochondria isolated in hypoxia compared to the mitochondria isolated in normoxia. 


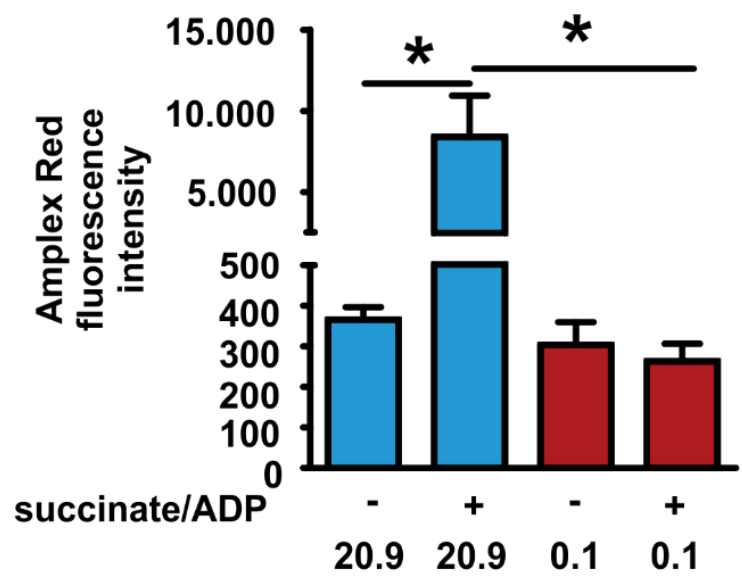

$\left(\% \mathrm{O}_{2}\right)$

Figure 20: Hydrogen peroxide production in mitochondria isolated at $20.9 \% \mathrm{O}_{2}$ compared to $\mathbf{0 . 1 \% ~} \mathrm{O}_{2}$. Mitochondria isolated from wild type mouse hearts ( $\mathrm{n}=3$ mice) in $20.9 \%$ $\mathrm{O}_{2}$ and $0.1 \% \mathrm{O}_{2}$ were incubated without or with $2.5 \mathrm{mM}$ succinate and $0.5 \mathrm{mM}$ ADP for $30 \mathrm{~min}$ in the respective oxygen concentration. Subsequently, mitochondria were analyzed for hydrogen peroxide levels with the help of the Amplex Red dye. Fluorescence intensity was calculated as mean $+/$ - SEM of individual experiments. ${ }^{*} p<0.05$.

\subsubsection{Complex III activity of mitochondria isolated in hypoxia versus normoxia}

Complex III in the mitochondrial ETC is one of the main producers of ROS. Since the data indicated less $\mathrm{O}_{2} \cdot$ and $\mathrm{H}_{2} \mathrm{O}_{2}$ levels in mitochondria isolated in $0.1 \% \mathrm{O}_{2}$, complex III activity was determined next. Mitochondria from wild type mouse hearts were isolated in $20.9 \%$ and $0.1 \%$ $\mathrm{O}_{2}$ and were subsequently tested for complex III activity. It was observed that complex III activity is significantly lower in mitochondria isolated in hypoxia as compared to normoxia (Fig. 21).

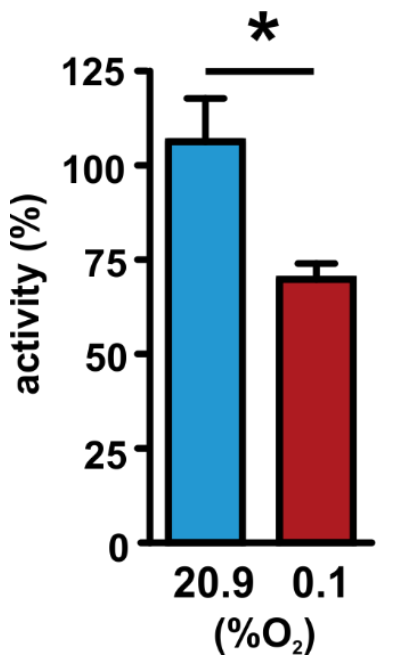

Figure 21: Activity of Complex III is less in mitochondria isolated in hypoxia. Mitochondria isolated from wild type mouse hearts ( $\mathrm{n}=3$ mice) in $20.9 \% \mathrm{O}_{2}$ and $0.1 \% \mathrm{O}_{2}$ were measured for complex III activity. Activity (\%) was calculated as mean $+/$ - SEM of individual experiments. * $p<0.05$. 


\subsubsection{ATP produced by mitochondria isolated in hypoxia versus normoxia}

Hypoxic isolated mitochondria possess a more reduced $E_{\mathrm{GSH}}$, less ROS production as well as lower complex III activity than normoxic isolated mitochondria. Therefore, I was analyzing the overall effects on ATP production of mitochondria isolated in $20.9 \% \mathrm{O}_{2}$ versus $0.1 \% \mathrm{O}_{2}$. Mitochondrial $\mathrm{Ca}^{2+}$ can stimulate higher ATP production. Mitochondria were isolated in $20.9 \%$ $\mathrm{O}_{2}$ and $0.1 \% \mathrm{O}_{2}$ from wild type mouse hearts. These mitochondria were incubated in buffer without or with $2.5 \mathrm{mM}$ succinate and $0.5 \mathrm{mM}$ ADP as well as without or with $10 \mu \mathrm{M} \mathrm{CaCl}_{2}$ as indicted in Fig. 22 for $30 \mathrm{~min}$ at the respective oxygen concentrations. Following incubation, mitochondria were centrifuged and the supernatants were used for the ATP assay. Strikingly, mitochondria isolated in $0.1 \% \mathrm{O}_{2}$ could produce higher ATP levels even without presence of any substrate like in this case succinate/ADP. In presence of succinate/ADP as well as $\mathrm{CaCl}_{2}$ the amount of ATP produced by the mitochondria isolated in hypoxia was significantly higher than by mitochondria isolated in normoxia. This data set indicates that mitochondria isolated in hypoxia not only have reduced $E_{\mathrm{GSH}}$, produce less ROS with lower complex III activity but also work more efficiently with regards to ATP production than the mitochondria isolated in normoxia.

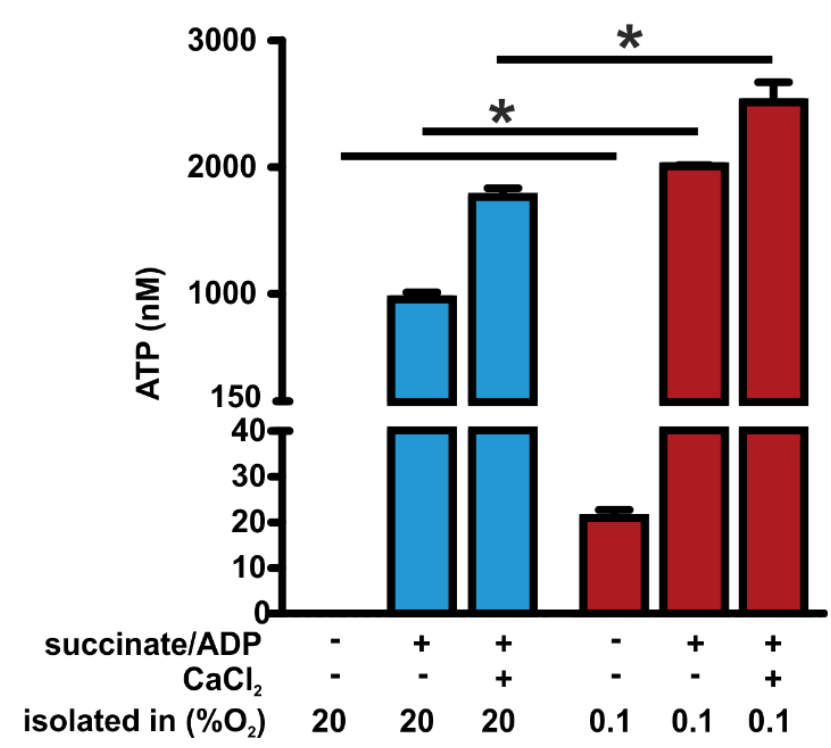

Figure 22: Higher amount of ATP is produced by the mitochondria isolated in hypoxia as compared to mitochondria isolated in normoxia. Mitochondria were isolated from wild type mouse hearts ( $\mathrm{n}=3$ mice) in $20.9 \% \mathrm{O}_{2}$ and $0.1 \% \mathrm{O}_{2}$ and incubated in buffer without or with $2.5 \mathrm{mM}$ succinate and $0.5 \mathrm{mM}$ ADP as well as without or with $10 \mu \mathrm{M} \mathrm{CaCl}_{2}$ as indicated for 30 $\mathrm{min}$ at the respective oxygen concentrations. The supernatants from the centrifuged mitochondria were used for measuring the ATP levels in each condition. ATP (nM) was calculated as mean $+/$ - SEM of individual experiments. * $p<0.05$. 


\subsubsection{Electron transport chain protein complexes are preserved in mouse cardiac mitochondria isolated in normoxia and hypoxia}

The data acquired so far indicated that mitochondria isolated in hypoxia behave differently than the mitochondria isolated in normoxia regarding ROS and ATP production. Therefore, I subsequently investigated the structural integrity with regards to ETC complexes in mitochondria isolated in normoxia versus hypoxia. Mitochondria from wild type mouse hearts were isolated in $20.9 \%$ and $0.1 \% \mathrm{O}_{2}$ and lysed in solubilization buffer. Solubilized mitochondria from each condition were loaded on to a Blue Native PAGE gel. ETC protein complex structures and levels were visualized in a subsequently performed Western blot by using antibodies against NDUFB8, Rieske and Cox1 for complex I, III and IV, respectively. VDAC3 was used as a protein loading control (Fig. 23a). As shown in Fig. 23b, there were no obvious differences detectable in the super complex formation within the different respiratory chain complexes as well as no difference in the ETC protein levels of the mitochondria isolated in normoxia versus hypoxia. These data suggest that there is nothing uncommon observed with respect to mitochondrial ETC complex structures in the mitochondria isolated in hypoxia.

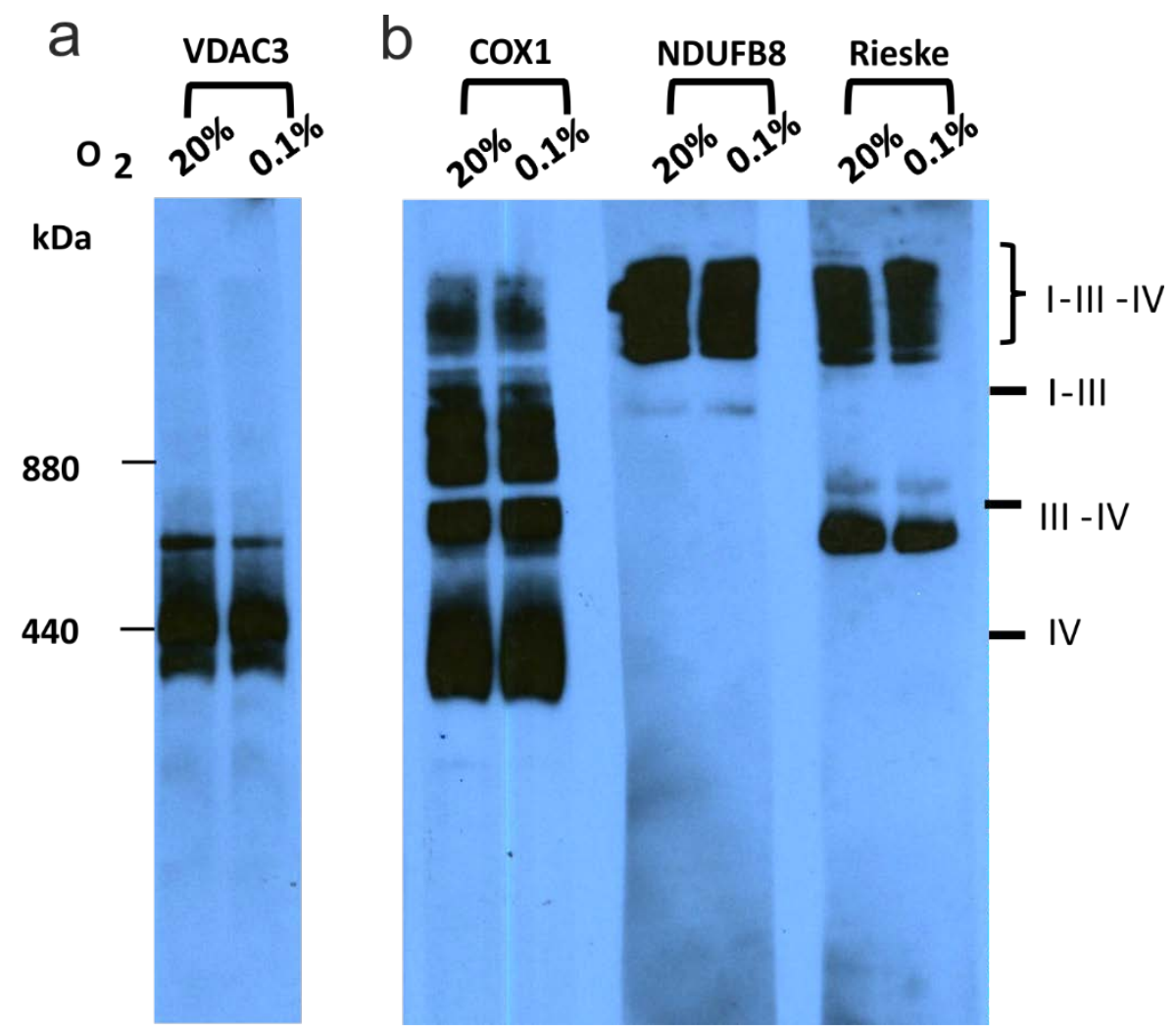

Figure 23: Mitochondria isolated in hypoxia and normoxia have similar ETC complex structures detected on Blue Native PAGE. Mitochondria isolated in $20.9 \% \mathrm{O}_{2}$ and $0.1 \% \mathrm{O}_{2}$ from a wild type mouse hearts were solubilized in the indicated oxygen concentrations and loaded on to a Blue Native gel. Subsequently, Western blots were performed and (a) VDAC3 was analyzed as protein loading control and (b) protein levels of complex I, III and IV by using the indicated antibodies were examined. 


\subsubsection{MCU of mouse cardiac mitochondria isolated in normoxia and hypoxia on a Blue Native PAGE}

As analyzed above, $\mathrm{Ca}^{2+}$ can trigger the mitochondria isolated in hypoxia to produce significantly higher ATP levels as compared to mitochondria isolated in normoxia. Based on this data set, I analyzed possible structural changes in the MCU complex in mitochondria isolated in hypoxia versus normoxia. Along the line of testing the mitochondrial ETC complexes isolated in normoxia and hypoxia, I tested MCU at protein level on a BN PAGE (Fig. 24). Mitochondria were isolated in normoxia and hypoxia from wild type mouse hearts, successively solubilized in the respective oxygen concentration and loaded on to the BN PAGE gel. VDAC3 was used as a protein loading control. The MCU complex appeared in three major bands (around $400 \mathrm{kDa}$, around $800 \mathrm{kDa}$ and $>800 \mathrm{kDa}$ ). The higher molecular weight MCU complex (>880 KDa) appeared more intense in mitochondria isolated in hypoxia indicating that aside from the functional changes analyzed above, oxygen likewise affects the structural arrangement of MCU.

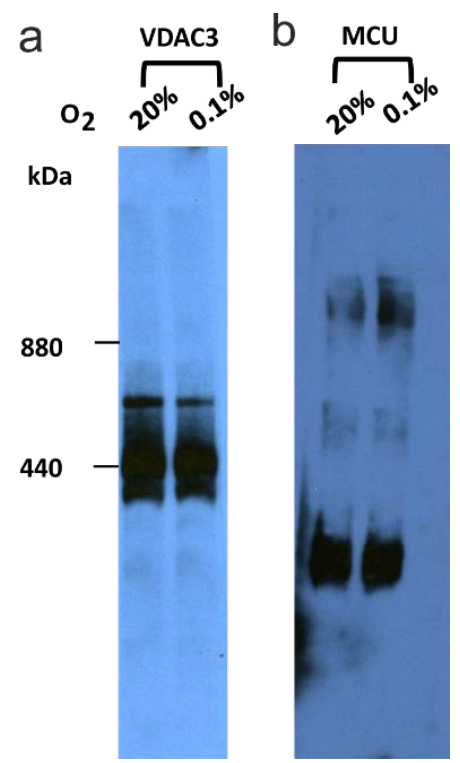

Figure 24: Mitochondria isolated in hypoxia arrange the MCU in a high molecular weight supercomplex. Mitochondria isolated in $20.9 \% \mathrm{O}_{2}$ and $0.1 \% \mathrm{O}_{2}$ from a wild type mouse were solubilized in the respective oxygen concentrations and loaded on to a Blue Native gel. Subsequently Western blots for (a) VDAC as protein loading control and (b) MCU as the protein of interest were performed.

\subsection{Generation of a DAAO-HyPer mouse model}

\subsubsection{DAAO-HyPer transgenic mice}

The third main goal of my thesis was to develop and characterize a mouse model, which would allow manipulating endogenous ROS levels. The main purpose behind developing this mouse model was to have an in vivo model which produces ROS internally in a physiologically 
acceptable concentration. To this end, I developed a mouse model having the fusion protein DAAO-HyPer in the cytoplasm and nucleus respectively, expressed under the activity of the $\alpha-$ MHC promoter. This transgenic mouse model comprises a fusion protein containing the enzyme DAAO as a $\mathrm{H}_{2} \mathrm{O}_{2}$ producer and HyPer as a biosensor to detect the produced $\mathrm{H}_{2} \mathrm{O}_{2}$. The DAAO-HyPer transgenic mice were generated by pronuclear blastocyst injections of the purified DNA into the C57BL/6N mice using standard procedures by the core facility of the Max-Planck Institute of Experimental Medicine, Göttingen. As shown in Fig. 25, we generated two independent transgenic mouse models consisting of the fusion protein located in the cytoplasm of the cardiomyocytes by using a nuclear export signal (NES) and in the nucleus by using a nuclear export signal (NLS).

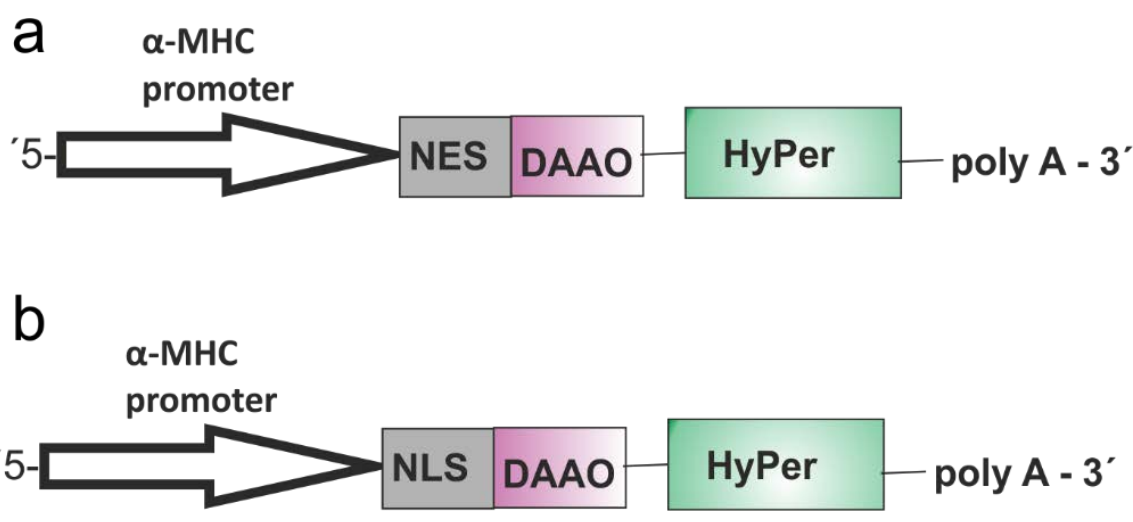

Figure 25: Schematic representation of the plasmids used for generation of cyto DAAOHyPer and nucleus DAAO-HyPer transgenic mouse models. (a) $\alpha-\mathrm{MHC}$ promoter driven DAAO-HyPer biosensor was located in the cytoplasm of the cardiomyocytes by nuclear export signal (NES) and (b) targeted to the nucleus with the help of nuclear localization signal (NLS).

\subsubsection{Confirmation of the DAAO-HyPer transgene by genotyping}

In order to confirm the presence of the expected transgene in the respective mouse line, genotyping was performed on tail biopsies by standard PCR. The forward and the reverse primers were designed in such a way that the forward primer binds to the region of the NLS and reverse primer binds in the coding region of DAAO. This set of primers helped to detect the nucleus DAAO-Hyper transgene. Similarly, in the case of cyto DAAO-HyPer transgene the forward primer binds to the NES region and the reverse primer binds to the coding region of DAAO. This makes it possible to detect each transgene independently. As shown in Fig. 26, tail biopsies from the cytoplasm DAAO-HyPer and nucleus DAAO-Hyper mouse line showed a PCR product at 280 bp whereas no band at 280 bp was observed in case of a wild type mouse. This was also confirmed by using the original plasmid DNA as positive control. 
a

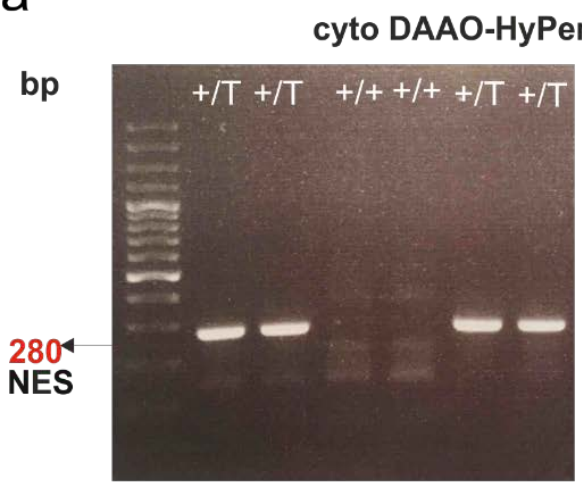

b

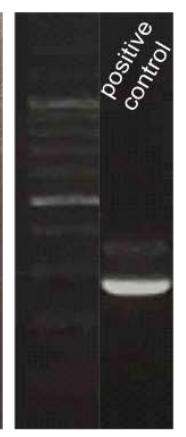

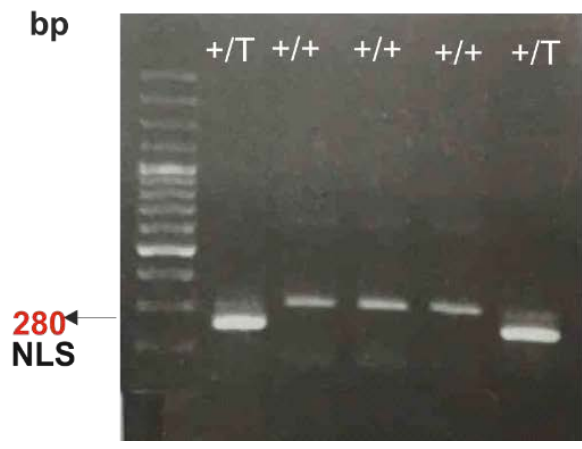

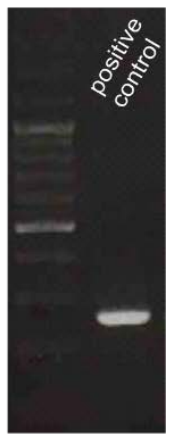

Figure 26: Genotyping results confirming the presence of the transgene in DAAO-HyPer NES and DAAO-Hyper NLS mice. (a) A 2\% agarose gel containing the PCR products showing the presence of nuclear export signal (NES) transgene (280 bp) in 6 different mice from the cyto DAAO-HyPer breeding and a positive control as a reference. (b) A $2 \%$ agarose gel containing the PCR products showing the presence a transgene nuclear localization signal (NLS) transgene (280 bp) in 5 different mice from the nucleus DAAO-HyPer breeding and a positive control as a reference.

\subsubsection{Selecting functionally positive founder lines for DAAO-HyPer NES and DAAO-HyPer NLS mice}

There were independent founder lines generated with the DAAO-HyPer NES and DAAOHyPer NLS plasmids namely DAAO-NES\#1, 4, 10, 11 and DAAO-NLS\#1, 3, 4, 9, 10. The first check point of confirming the presence of the transgene in these particular mice was performed by genotyping as shown above. Subsequently, from a minimum of two mice from each of the founder lines, cardiomyocytes were isolated by Langendorff's perfusion system. These cardiomyocytes were screened for two characteristics, namely presence of a HyPer fluorescence signal in the respective cellular compartment and response of the DAAO enzyme to its substrate D-alanine. During this phase of characterization, I observed that especially in the cardiomyocytes isolated from the DAAO-NES mice, the HyPer was expressed; however the expression was so low that it could not give any visual response to D-alanine. In case of the DAAO-NLS mouse lines, there was only one positive founder line among all the lines tested, which fulfilled the above described criteria (Fig. 27). This founder line was the DAONLS\#9 line, which not only showed a clear presence of the HyPer signal in the nucleus but also DAAO enzyme responded to the substrate D-alanine. 
a-MHC DAAO-HyPer NES founder lines

- DAAONESHI

- taagnesha

- maAOnesh10

- taAanesh1
a-MHC DAAO-HyPer NLS founder lines

- DAAONESHI

- taAG NLSH3

- taagntsha

DAAO NLS\#9

\section{- taAAONESHio}

Figure 27: DAO-NLS\#9 was found to be the only positive founder line from all the DAAONES and the DAAO-NLS mice generated. In order to characterize all the founder lines generated in the DAAO-HyPer NES and mito DAAO-HyPer NLS mouse lines, cardiomyocytes isolated from each of the lines were imaged and measured using an inverted epifluorescence microscope IX83 (Olympus).

\subsection{Epifluorescence microscopic image of the cardiomyocytes isolated from DAAO-HyPer NLS\#9}

Cardiomyocytes were isolated from nucleus DAAO-HyPer NLS\#9 mice by Langendorff's perfusion system. The cardiomyocytes were imaged using an inverted epifluorescence microscope. HyPer probe was excited at 420 and $500 \mathrm{~nm}$ and the emitted light was detected by a CCD camera at $510 \mathrm{~nm}$. As shown in Fig. 28, the cardiomyocytes possess in their nuclei a strong DAAO-HyPer signal both at 420 and $500 \mathrm{~nm}$ confirming the presence of the biosensor in the nucleus.

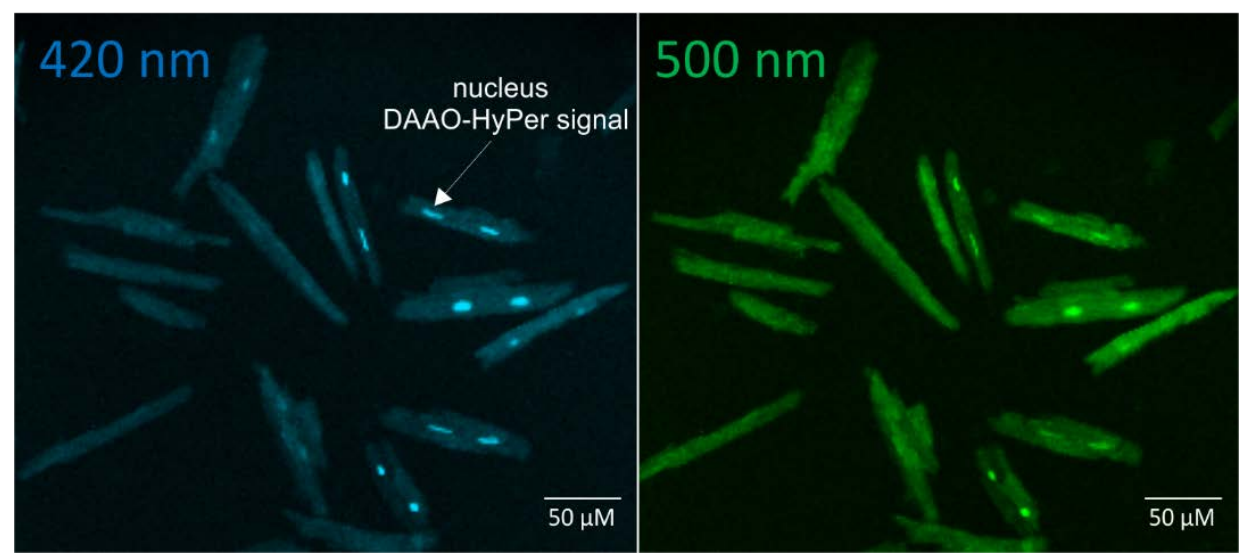

Figure 28: Epifluorescence microscopic image of cardiomyocytes expressing DAAOHyPer fusion protein in the nucleus. Cardiomyocytes isolated from a nucleus DAAO-HyPer transgenic mouse by Langendorff' s perfusion system were observed for the presence of the expected HyPer based fluorescence signal in the nucleus. Imaging was performed using the inverted epifluorescence microscope IX83 (Olympus). HyPer probe was excited at 420 and $500 \mathrm{~nm}$ and the emitted light was detected by a CCD camera at $510 \mathrm{~nm}$. 


\subsection{Echocardiography of wild type versus transgenic nucleus DAAO-HyPer biosensor mice}

The nucleus DAAO-HyPer mice are also monitored over time for their heart function by echocardiography. Fig. 29 indicates the FAS and the EF in transgenic mice versus wild type mice after every 4 weeks respectively. It can be seen that there is no reduction in the heart function parameters of the transgenic mice as compared to wild types. This suggests that the DAAO-HyPer biosensor itself is not compromising cardiac function.

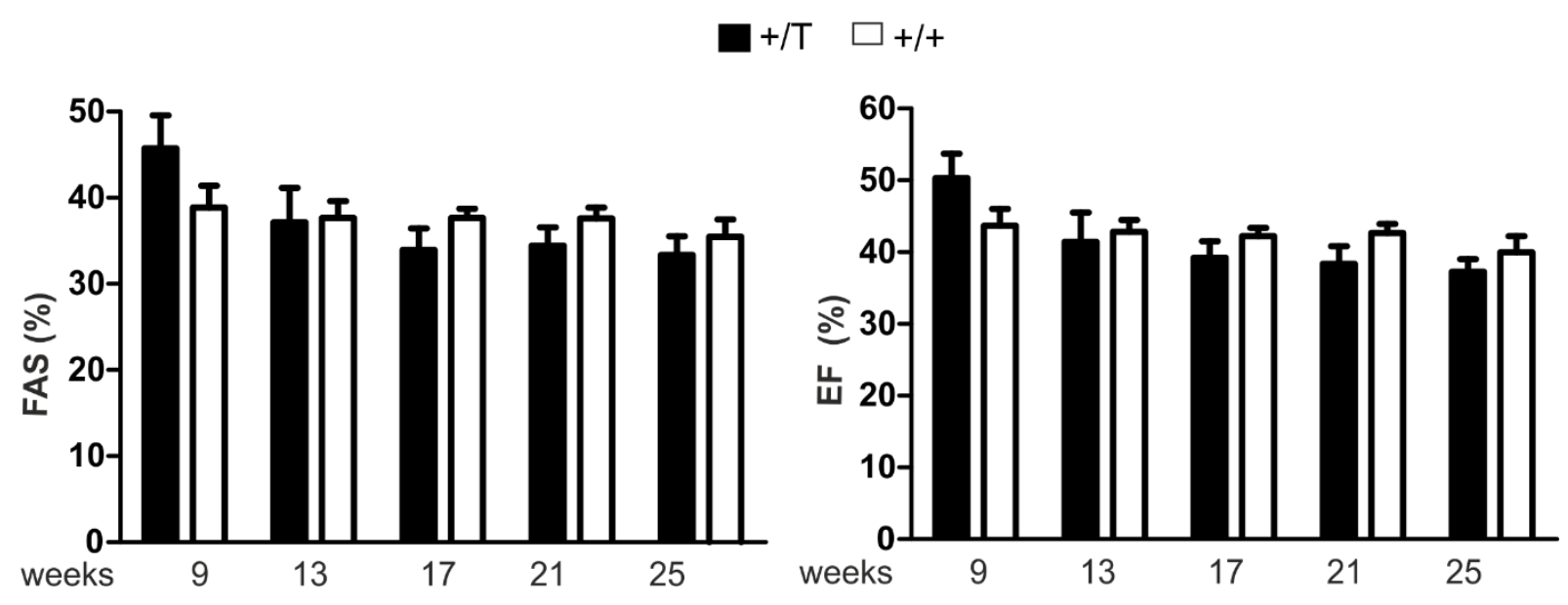

Figure 29: Nucleus DAAO-HyPer transgenic mice have normal heart function. Cardiac function of transgenic ( $n=3$ mice, $+/ T)$ and wild type $(n=5$ mice, $+/+)$ Nucleus DAAO-HyPer mice were recorded for heart functioning by echocardiography for every 4 weeks from 9 weeks of age on. Fractional Area Shortening (FAS) and Ejection Fraction (EF) of these mice were recorded till the age of 25 weeks.

\subsection{In vitro stimulation of cardiomyocytes isolated from nucleus DAAO- HyPer mouse by D-alanine}

The presence of HyPer in the nucleus of DAAO-HyPer cardiomyocytes was confirmed through microscopy. However, to confirm the presence of the enzyme DAAO, cardiomyocytes were treated with its substrate D-alanine to activate the enzyme. Upon activation of DAAO, there should be a particular amount of $\mathrm{H}_{2} \mathrm{O}_{2}$ produced which in turn should be detectable by the fused HyPer probe. In order to confirm this, the following experiment was performed. Cardiomyocytes were isolated from transgenic nucleus DAAO-Hyper mice by Langendorff's perfusion system. The cardiomyocytes were used for live time imaging using an inverted epifluorescence microscope. During live time imaging, the cardiomyocytes were stimulated with various concentrations of $D$-alanine ranging from $3 \mathrm{mM}$, to $10 \mathrm{mM}$. Subsequently, before and after addition of the respective D-alanine concentrations, the changes in the fluorescence intensities of the HyPer probe at 420 and $500 \mathrm{~nm}$ excitation wavelengths were recorded. The changes in the fluorescence intensities from each excitation wavelengths over the entire 
measurement time was plotted in order to observe the effect of addition of D-alanine on the enzyme DAAO recorded by the HyPer probe. As shown in Fig. 30, there is a stable base line obtained before addition of any D-alanine, without any changes in the 420 and $500 \mathrm{~nm}$ wavelengths. Once the D-alanine is added, DAAO gets activated and produces $\mathrm{H}_{2} \mathrm{O}_{2}$. HyPer in turn measures the extent of $\mathrm{H}_{2} \mathrm{O}_{2}$ produced by the DAAO. A typical response after addition of $D$-alanine shows an increase in $500 \mathrm{~nm}$ wavelength and a decrease in $420 \mathrm{~nm}$ wavelength. The changes in the fluorescence intensities recorded by HyPer for each concentration of Dalanine are observed to be a dose-dependent. The extent of the change in fluorescence intensities increases with increasing D-alanine concentrations. At the end of the stimulation with D-alanine, $100 \mu \mathrm{M} \mathrm{H}_{2} \mathrm{O}_{2}$ are added to achieve full oxidation. At this stage, the changes in the fluorescence intensities are observed to reach their highest extent which indicates that the cardiomyocytes are then fully oxidized.

$10 \mathrm{mM}$ D-alanine

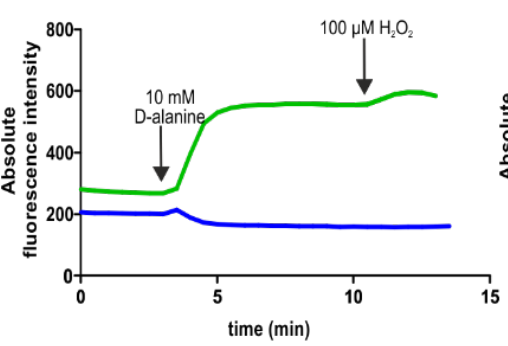

4 mM D-alanine

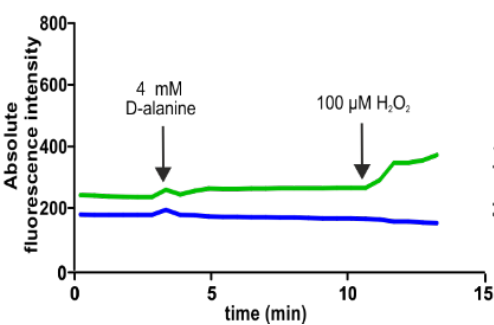

$8 \mathrm{mM}$ D-alanine
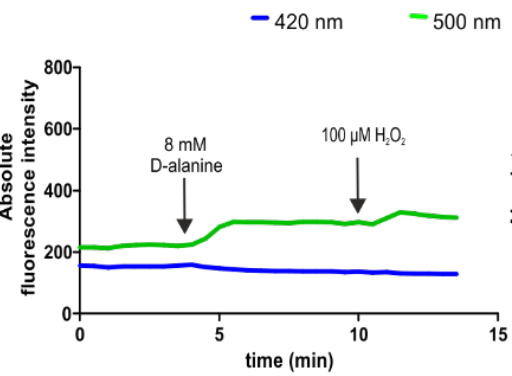

$3 \mathrm{mM}$ D-alanine

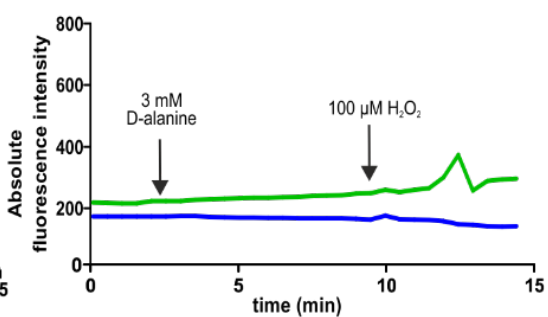

6 mM D-alanine

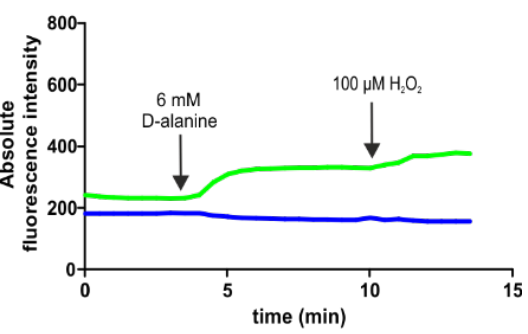

Figure 30: Cardiomyocytes isolated from nucleus DAAO-HyPer mice respond in a dose dependent manner when titrated with various $D$-alanine concentrations. Cardiomyocytes isolated from transgenic nucleus DAAO-HyPer mice hearts $(n=3$ mice) were treated with 10 , $8,6,4$ and $3 \mathrm{mM}$ of D-alanine respectively. The changes in fluorescence intensities of 420 and $500 \mathrm{~nm}$ wavelengths were recorded using an epifluorescence microscope. After reaching a plateau upon treatment with D-alanine, $100 \mu \mathrm{M} \mathrm{H}_{2} \mathrm{O}_{2}$ was added to the cardiomyocytes to achieve a complete oxidation response.

\subsection{In vitro stimulation of cardiomyocytes isolated from nucleus DAAO- HyPer mouse by D-alanine versus L-alanine}

As a control for activation of the DAAO enzyme, cardiomyocytes were also stimulated with 10 $\mathrm{mM}$ L-alanine. Unlike D-alanine, L-alanine should not activate cardiomyocytes and there 
should be no changes observed in the fluorescence intensities of the excitation wavelengths of 420 and $500 \mathrm{~nm}$ after addition of L-alanine. Fig. 31 shows a titration curve representing the normalized ratio of $420 / 500 \mathrm{~nm}$ on stimulation of cardiomyocytes with different concentration of D-alanine and the highest concentration of L-alanine. It's seen that the titration curve obtained from the increasing concentration of D-alanine gives a dose-dependent response whereas, with the highest concentration of L-alanine does not induce any response. This confirms (i) the functionality and (ii) the specificity of the DAAO enzyme in the transgenic mice.

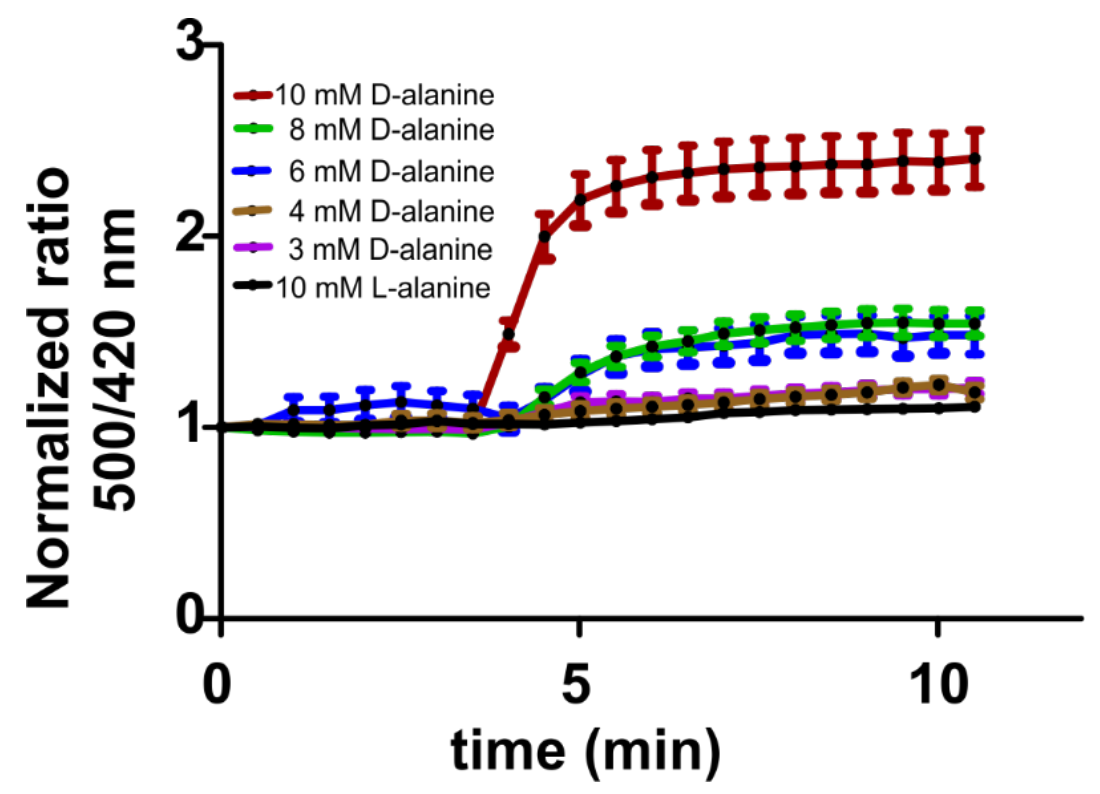

Figure 31: Cardiomyocytes isolated from nucleus DAAO-HyPer mice respond in a dose dependent manner when titrated with various $D$-alanine concentrations but not to Lalanine. Normalized ratio of fluorescence excitations at 500/420 nm after stimulation of cardiomyocytes isolated from DAAO-HyPer mice hearts with 10, 8, 6, 4 and $3 \mathrm{mM}$ of D-alanine and $10 \mathrm{mM}$ of L-alanine. 


\section{Discussion}

ROS when produced at low amounts in any biological system serve as regulators of various signaling pathways. However, if produced in high amounts they can be lethal to cells (Patel et al., 2018). To maintain a fine balance in the cellular redox status, cells possess an antioxidant defense system to buffer ROS. The Glutaredoxin (Grx) antioxidant defense system is one of the major system playing a role in this regard (Fernandes and Holmgren, 2004). While Grx is working as an antioxidant, the process shapes the $E_{\mathrm{GSH}}$ inside the cell (Millis et al., 1993). In order to study redox signaling pathways and associated cellular functions, it is essential to have basic tools to precisely measure the $E_{\mathrm{GSH}}$ in cells.

A wide range of synthetic probes are commonly used for measuring several ROS produced in any kind of biological system. However, the use of these chemical sensors is associated with their own advantages and disadvantages (Kalyanaraman et al., 2012). As an attempt to precisely measure the $E_{\mathrm{GSH}}$, the first main objective of my thesis was to characterize a genetically encoded Grx1-roGFP2 redox biosensor mouse model which then was further utilized to study redox-dependent processes especially in cardiomyocytes.

\subsection{Ageing affects the redox compartmentalization in cardiomyocytes}

\subsubsection{Redox compartmentalization in isolated cardiomyocytes from Grx1- roGFP2 mice}

In the Institute of Cardiovascular Physiology, University Medical Center Göttingen, cardiomyocyte specific transgenic mouse models expressing the Grx1-roGFP2 redox biosensor were previously developed by Dr. Lija Swain (Swain et al., 2016). In these mouse models, the redox biosensor is located in two different compartments of the cardiomyocytes namely in the cytoplasm and the mitochondrial matrix. Taking advantage of these mouse models, I chose to characterize both mouse models with respect to their $E_{\mathrm{GSH}}$ in the respective compartment. The biosensor comprises the fusion protein Grx1 coupled to roGFP2. This combination allows a real time visualization of the $E_{\mathrm{GSH}}$ in the specific compartment. The probe has two excitation wavelengths at 405 and $488 \mathrm{~nm}$ (Dooley et al., 2004). Obtaining ratiometric values instead of a single value makes the measurements more reliable and specific.

While investigating the differences of the redox status between cytoplasm and mitochondrial matrix of cardiomyocytes, $\mathrm{H}_{2} \mathrm{O}_{2}$ or diamide and DTT were used as oxidizing and reducing agents. Cardiomyocytes were isolated from each of the transgenic mice by Langendorff's perfusion system. The isolated cardiomyocytes were imaged using epifluorescence microscopy. The roGFP2 probe was excited at 405 and $488 \mathrm{~nm}$. It was observed that when 
the oxidizing agent was added to the cardiomyocytes containing the biosensor in the cytoplasm, the cytoplasm had a very low capacity to oxidize further. Therefore, there were no major changes observed in the fluorescence intensities at 405 and $488 \mathrm{~nm}$ upon addition of $\mathrm{H}_{2} \mathrm{O}_{2}$ or diamide. However, when $\mathrm{H}_{2} \mathrm{O}_{2}$ or diamide were added to the cardiomyocytes containing the biosensor in the mitochondrial matrix, there was a significant change observed in the fluorescence intensities of the roGFP2 excitation wavelengths. Notably, this shows that the mitochondrial matrix has a higher capacity to get oxidized, which implies the original redox status is indeed highly reduced. On the other hand, the cytoplasm has lower capacity to oxidize which in turn depicts its already highly oxidized redox nature.

These data signify the difference between the $E_{\mathrm{GSH}}$ of the cytoplasm and the mitochondrial matrix in cardiomyocytes. That implies that there is a redox compartmentalization present in cardiomyocytes. This might justify that the two compartments have independent redox regulations, which are reflected by their specific cellular functions. For instance, the mitochondrial matrix serves as a site for the respiratory chain activity. ETC involves a fine tuned network between different players like NADH and NADPH as reducing equivalents, complex I and III as main ROS generators and GSH as a redox buffer. In contrast, the cytoplasm has a completely different role in the cell.

The concept of cellular redox compartmentalization was also discussed earlier in other studies. In one of the studies which were done in yeast with the help of a peroxi-redoxin based sensor, it was observed that the cytosol and the mitochondrial matrix possess varied capacities to hold $\mathrm{H}_{2} \mathrm{O}_{2}$ in that compartment (Morgan et al., 2016). The authors demonstrated that the respiratory chain activity does not exert any effect on the cytoplasmic $\mathrm{H}_{2} \mathrm{O}_{2}$. In contrast, the cytoplasmic $\mathrm{H}_{2} \mathrm{O}_{2}$ does affect the respiratory chain activity in the mitochondrial matrix. A similar phenomenon was observed in a Drosophila study, which included the use of Grx1-roGFP2 and Orp1 based $E_{G S H}$ and $\mathrm{H}_{2} \mathrm{O}_{2}$ probes in the cytosol and mitochondrial matrix (Albrecht et al., 2011). In this study, it was reported that the cytosol and the mitochondrial matrix possess $E_{\mathrm{GSH}}$ which are independent from each other. This observation also pointed out that there was no notable correlation between the $E_{\mathrm{GSH}}$ of the individual compartments with the corresponding $\mathrm{H}_{2} \mathrm{O}_{2}$ levels. Altogether, this signifies that the thioredoxin-coupled peroxidases metabolizing $\mathrm{H}_{2} \mathrm{O}_{2}$ and the glutaredoxin based antioxidant defense system buffering GSSG are independent.

\subsection{2. $E_{\mathrm{GSH}}$ of isolated cardiomyocytes from cyto and mito Grx1-roGFP2 aged mice}

While characterizing the cyto and mito Grx1-roGFP2 mice, it was tempting to also use these mouse models to study the effect of aging on the $E_{G S H}$ of cardiomyocytes. There are many 
studies reported regarding the correlation between redox regulation and aging (Liguori et al., 2018). However in the past, different studies approached the question in a different manner and ended up in at least in part conflicting results. Some studies demonstrated that mitochondrial oxidative stress is beneficial and can increase the life span in mice (Hekimi, 2013). On the contrary, some reports showcase that mitochondrial damage and dysfunction are observed in aged cells (Picard et al., 2010). This damage includes gradual decrease in the respiration capacity of the mitochondria, decreased activity of ETC complexes, oxidative damage and fragility of aged mitochondria.

In the presented study, the Grx1-roGFP2 mouse models were used to quantitate the $E_{G S H}$ in isolated cardiomyocytes in young versus old mice. Since the biosensor is present in the cytoplasm and the mitochondrial matrix of the cardiomyocytes, it was possible to analyze the changes in the $E_{\mathrm{GSH}}$ of subcellular compartments in aging mice. Cardiac function of the mice was recorded over time by echocardiography in order to observe a possible decrease in the heart function upon aging. At the age of 80 weeks the heart function was recorded to be declined compared to younger mice. Therefore, the 80 weeks old mice were chosen to isolate cardiomyocytes and measure the $E_{\mathrm{GSH}}$ in each compartment. It was observed that the $E_{\mathrm{GSH}}$ of the mitochondrial matrix does not change during aging and stays to be naturally reduced, similar to that of young mice. However, the $E_{G S H}$ of the cytoplasm gets further reduced compared to the cytoplasm of the cardiomyocytes of younger mice which was seen to be oxidized. Thus, the $E_{\mathrm{GSH}}$ recorded in young mice showed a redox compartmentalization within the cardiomyocytes. However, with aging this seems to disappear. The impact of this loss in compartmentalization still needs to be analyzed in further depth, especially if this is the consequence or cause of aging.

\subsection{Oxygen affects the mitochondrial functionality ex vivo 4.2.1. Redox histology of the cardiac sections using mito Grx1-roGFP2 mice}

As described above, the OxD and in line the $E_{\mathrm{GSH}}$ of the mitochondrial matrix in isolated cardiomyocytes were seen to be more reduced compared to the cytoplasm. During the isolation process, cardiomyocytes are taken out of the tissue. In consequence cells are losing contact to their neighboring cells and are exposed to a different microenvironment. In order to mimic the physiological conditions better, the OxD of the mitochondrial matrix was therefore also analyzed using cardiac sections from the mito Grx1-roGFP2 mice. The OxD of the cardiac sections when treated with NEM was comparable to the $E_{\mathrm{GSH}}$ of the mitochondrial matrix of the isolated cardiomyocytes. However, there was an increased oxidation observed when the sections were not incubated in NEM. This gives a hint that it is important for the mitochondrial matrix to be in its physiological environment for maintaining its reduced status. This can also 
be related to the fact that cardiac sections cannot stay intact after the freezing process and this might affect cellular antioxidant mechanisms. The obtained oxidized values in the untreated cardiac sections might be also the result from the oxidation of the sensor itself and due to the fixation steps. Most interestingly, the OxD of the mitochondrial matrix in isolated cardiomyocytes with or without NEM treatment did not show any difference demonstrating that unlike cardiac sections, the intact cardiomyocytes are able to maintain their $E_{\mathrm{GSH}}$.

\subsubsection{EGSH of isolated mitochondria in normoxia versus hypoxia}

As discussed in the earlier section, each organelle has its own independent $E_{G S H}$ probably due to the differences in their role for cellular functions. What happens to the $E_{G S H}$ of the mitochondrial matrix when mitochondria are isolated from cardiomyocytes is not well understood. NEM has the ability to block thiol groups and thus can freeze their redox status. Using this feature of NEM, it was used to freeze the redox status of the mitochondria during the entire mitochondrial isolation procedure. When the $E_{\mathrm{GSH}}$ of the mitochondria isolated from the mitoGrx1-roGFP2 mouse model were analyzed, it was observed that the $E_{\mathrm{GSH}}$ of the mitochondria isolated without NEM was completely oxidized almost up to $100 \%$ compared to a roughly $45 \%$ oxidation in the intact cardiomyocytes. To find out the reason behind the change in the $E_{\mathrm{GSH}}$ of mitochondria during isolation, in the subsequent experiments the hypothesis that the oxygenation plays a role, was tested. The $\mathrm{pO}_{2}$ reaching the mitochondrial matrix when placed inside a cell versus when being isolated in the atmospheric environment is significantly different (Keeley and Mann, 2018; Kurtcuoglu et al., 2015). With this thought in mind, I proceeded isolating mitochondria in low oxygen concentrations starting from $10 \% \mathrm{O}_{2}$ and went to as low as $0.1 \% \mathrm{O}_{2}$. Subsequently, the $E_{\mathrm{GSH}}$ of mitochondria isolated in $20.9 \% \mathrm{O}_{2}$ and 0.1 $10 \% \mathrm{O}_{2}$ were compared. Strikingly, the idea of molecular oxygen being involved as one of the factors to maintain the redox status of the mitochondrial matrix held some hidden truth with it. The mitochondria isolated in hypoxia had maintained the mitochondrial reduced $E_{\mathrm{GSH}}$, which was very close to the $E_{G S H}$ of the mitochondrial matrix inside the isolated cardiomyocytes. The mitochondrial matrix was successful in maintaining the reduced $E_{\mathrm{GSH}}$ when exposed to $10 \% \mathrm{O}_{2}$ or less during the isolation process. More than $10 \% \mathrm{O}_{2}$ seems to be the threshold oxygen concentration wherein the mitochondria cannot preserve their mitochondrial $E_{\mathrm{GSH}}$.

The $\mathrm{pO}_{2}$ at ambient air (at sea level), organ and cellular levels is highly different under physiological conditions. Fig. 32 shows a schematic representation of the $\mathrm{pO}_{2}$ gradient from the atmospheric air to mitochondria inside the cell. 


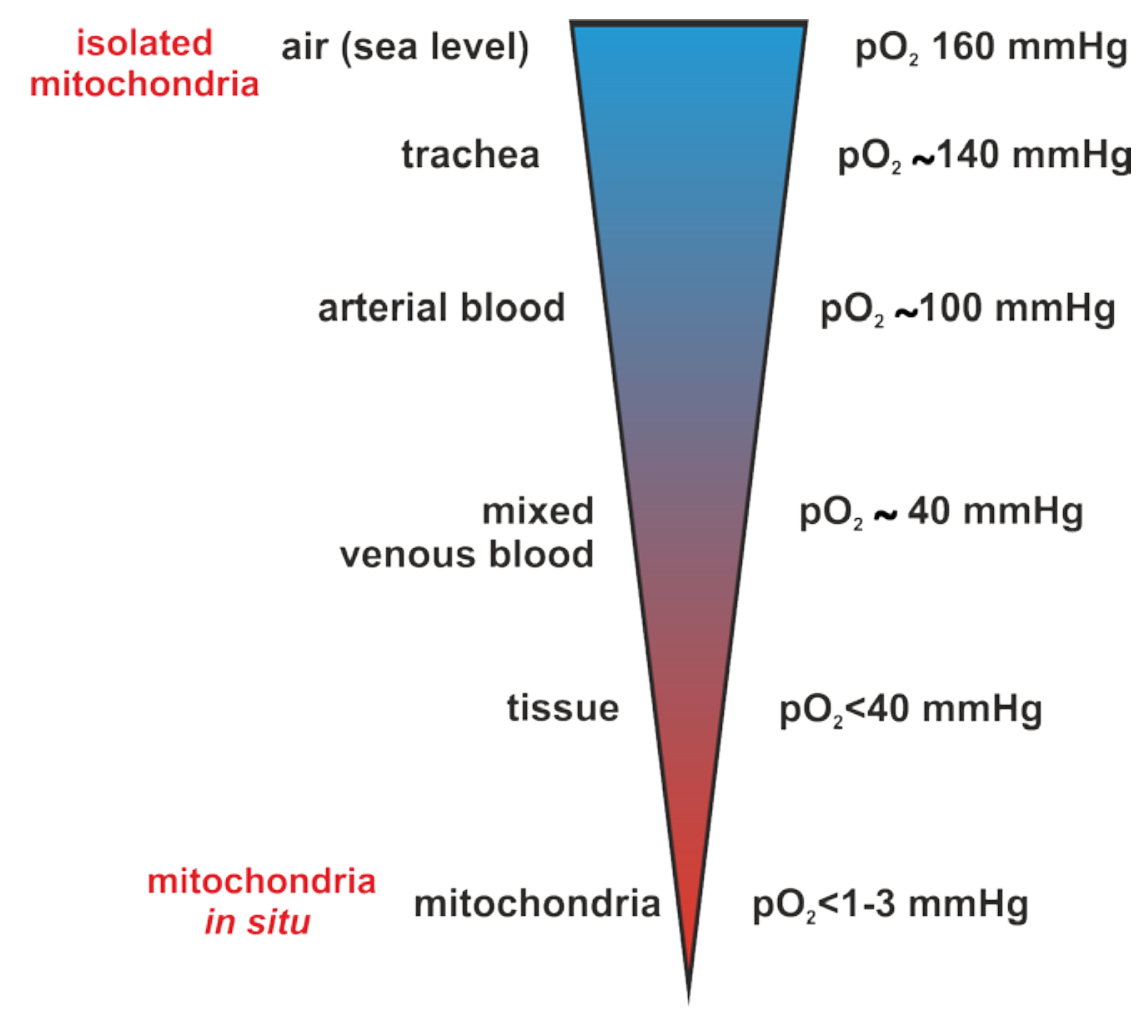

Figure 32: Schematic representation of the partial oxygen pressure $\left(\mathrm{pO}_{2}\right)$ gradient experienced at sea level to mitochondria present inside a cell.

The $\mathrm{pO}_{2}$ at sea level is as high as $160 \mathrm{~mm} \mathrm{Hg}$. However, when the air is inhaled and absorbed into the arterial blood passing via the trachea, the $\mathrm{pO}_{2}$ drops to roughly $100 \mathrm{~mm} \mathrm{Hg}$. As we go deep into tissue level and further to the mitochondria level, the $\mathrm{pO}_{2}$ drops as low as 1-3 mm $\mathrm{Hg}$ (Keeley and Mann, 2018; Kurtcuoglu et al., 2015). Therefore mitochondria, that are isolated at ambient air conditions, are exposed to a sudden rise in the oxygenation. This huge difference between the $\mathrm{pO}_{2}$ experienced by mitochondria isolated in normoxic conditions versus the mitochondria isolated in lower $\mathrm{pO}_{2}$ might explain at least in part the distinct $E_{\mathrm{GSH}}$ measurements between mitochondria isolated in normoxia versus hypoxia. When the mitochondria isolated in the hypoxic conditions were allowed to reoxygenate at normoxic conditions, the $E_{\mathrm{GSH}}$ of the mitochondria seemed to completely get oxidized. This shows that the $E_{\mathrm{GSH}}$ of mitochondria completely switches from a reduced to an oxidized status when they are incubated in a higher oxygen concentration. However when the normoxic isolated mitochondria were incubated in hypoxic conditions for 30 minutes, the $E_{\mathrm{GSH}}$ of mitochondria didn't get reduced. This shows that the oxidation reaction that occurs is irreversible and once the GSH pool has got already oxidized cannot revert back to its reduced form. 


\subsubsection{EGSH of isolated and energized mitochondria in normoxia versus hypoxia}

The $E_{G S H}$ of isolated mitochondria where the mitochondria were stimulated with substrates which help them to produce more ATP was analyzed. The addition of citric acid cycle substrates like succinate/ADP energizes mitochondria. In a recent study, done on isolated mitochondria from brain and liver it was shown that when isolated mitochondria were stimulated with substrates like glutamate and malate along with ADP, the GSH pool was more reduced compared to non-energized conditions (Garcia et al., 2010). The authors could additionally show an increase in the NADPH/NADP ratio upon stimulation of isolated mitochondria with these substrates. These data suggest that addition of substrates to mitochondria stimulates the regulation of the mitochondrial redox status which involves generation of more NADPH as reducing equivalents (Blacker and Duchen, 2016). These reducing equivalents can be further utilized by the GSSG reductase to convert GSSG to GSH as well as the recovery of S-glutathionylated proteins back to their reduced form by the glutaredoxin system (Holmgren et al., 2005).

In my experimental set up, I stimulated the mitochondria with succinate/ADP and utilized the mitochondria isolated from the mito Grx1-roGFP2 mouse model to visualize the changes in the $E_{\mathrm{GSH}}$ of the energized mitochondria. It was clearly observed that the mitochondria isolated in normoxia when stimulated with succinate have a reduced $E_{G S H}$ as compared to non-energized mitochondria. Moreover, the stimulated mitochondria isolated in lower oxygen concentrations had a significantly higher reduction in the $E_{\mathrm{GSH}}$ of the mitochondrial matrix than the stimulated mitochondria isolated in normoxia. This observation signifies the relation of a reduced $E_{\mathrm{GSH}}$ to a higher concentration of GSH present in the environment. This high concentration could have most probably derived from the increase in the concentration of reducing equivalents like $\mathrm{NADPH}$. Succinate gives rise to a higher concentration of NADH which in turn can generate $\mathrm{NADPH}$ by the nicotinamide nucleotide transhydrogenase and malic enzymes (Yap et al., 2009). Upon re-oxygenation of the mitochondria and then stimulating with the substrates, mitochondria reverted back to the $E_{\mathrm{GSH}}$ similar to the mitochondria isolated and stimulated in normoxia. This shows that the mitochondria when brought to a normoxic environment got oxidized compared to the hypoxic isolated mitochondria. In this condition, it can be seen that stimulated mitochondria were able to enhance the production of the required reducing equivalents however, at the same time the higher oxygen concentration disturbed their $E_{\mathrm{GSH}}$ status. 


\subsubsection{ROS levels and complex III enzyme activity in isolated mitochondria isolated in $20.9 \% \mathrm{O}_{2}$ versus $0.1 \% \mathrm{O}_{2}$}

After unraveling the fact that the oxygen levels affect the $E_{\mathrm{GSH}}$ of the mitochondrial matrix, the next obvious question coming up was about the ROS levels in mitochondria isolated in normoxia versus hypoxia. MitoSOX dye was used to analyze the mitochondrial ${ }^{\circ} \mathrm{O}_{2}{ }^{-}$produced in the mitochondria isolated in normoxia and hypoxia. The positively charged, lipophilic triphenylphosphonium group of mitoSOX having $\mathrm{a}^{\circ} \mathrm{O}_{2}{ }^{-}$sensitive dihydroethidium can enter the mitochondrial matrix (Roelofs et al., 2015).

In this assay, mitochondria were also stimulated with the substrate succinate/ADP to analyze the difference of ${ }^{\circ} \mathrm{O}_{2}{ }^{-}$produced between the energized and non-energized mitochondria. It was observed that the ${ }^{\circ} \mathrm{O}_{2}{ }^{-}$produced by the non-stimulated mitochondria isolated in hypoxia were significantly lower than the ${ }^{\circ} \mathrm{O}_{2}{ }^{-}$produced by non-stimulated mitochondria isolated in normoxia. As described above, mitochondria isolated in hypoxia have a reduced $E_{\mathrm{GSH}}$. There is certainly a strong correlation between the $E_{\mathrm{GSH}}$, the reducing equivalents and the produced ${ }^{\circ} \mathrm{O}_{2}{ }^{-}$. This is line with the fact that most of the ${ }^{\circ} \mathrm{O}_{2}{ }^{-}$are converted to $\mathrm{H}_{2} \mathrm{O}_{2}$ by SOD (Fukai and Ushio-Fukai, 2011). GSH is a redox buffer and can quickly scavenge a portion of ${ }^{\circ} \mathrm{O}_{2}{ }^{-}$as well as the converted $\mathrm{H}_{2} \mathrm{O}_{2}$ (Ighodaro and Akinloye, 2017).

Other than reduced redox nature which can explain the correlation to less ROS production, one of the next links in the tread of events was speculated to be the complex III activity. Complex III is known to produce leaky electrons which in turn combine with the molecular oxygen to generate ${ }^{\circ} \mathrm{O}_{2}{ }^{-}$(Chen et al., 2003). Keeping this in mind, the activity of complex III in mitochondria isolated in normoxia versus hypoxia was checked. It was observed that the mitochondria isolated in hypoxia have significantly lower complex III activity than the mitochondria isolated normoxia. Complex III transfers the electrons from CoQ to cytochrome c through a Q-cycle (Murphy, 2008). As lower complex III activity, as lower are the leaky electrons produced. In line, along with the lower ${ }^{\circ} \mathrm{O}_{2}{ }^{-}$levels, a lower complex III activity was observed.

When the ${ }^{\circ} \mathrm{O}_{2}{ }^{-}$levels with or without stimulating the mitochondria with succinate/ADP were analyzed, there were no major differences recorded comparing mitochondria which were energized or non-energized. When the mitochondria are stimulated, there are theoretically more electrons passing through the ETC. This would also mean high chances of leaky electrons. However, this was not observed in the performed experiments. ${ }^{\circ} \mathrm{O}_{2}{ }^{-}$are highly unstable. These ROS are very rapidly converted to $\mathrm{H}_{2} \mathrm{O}_{2}$ by SOD. This could be one of the reasons the MitoSOX dye could not detect any differences in the non-energized versus energized mitochondria. For this reason, the $\mathrm{H}_{2} \mathrm{O}_{2}$ levels generated in the mitochondria 
isolated in normoxia versus hypoxia were checked by using amplex Ultrared dye. Amplex red can detect $\mathrm{H}_{2} \mathrm{O}_{2}$ using HRP as a catalyst. In this reaction, the amplex red dye is oxidized to the fluorescent product resorufin by $\mathrm{H}_{2} \mathrm{O}_{2}$ and HRP. The extent of the fluorescence depicts the extent of the $\mathrm{H}_{2} \mathrm{O}_{2}$ present (Starkov et al., 2002). As expected, there was a higher amount of $\mathrm{H}_{2} \mathrm{O}_{2}$ detected in the energized mitochondria isolated in normoxia than in the non-stimulated ones. On the contrary, the $\mathrm{H}_{2} \mathrm{O}_{2}$ levels detected in the energized and non-energized mitochondria isolated in hypoxia were not demonstrating any major differences. This again correlates to the earlier observed link between reduced $E_{\mathrm{GSH}}$, higher reducing equivalents, less complex III activity and thus less ROS detection in the mitochondria isolated in hypoxia.

\subsubsection{ATP production by the mitochondria isolated in normoxia versus hypoxia.}

As discussed above I could observe a strong correlation between the oxygen levels the mitochondria are exposed to and their functionality. However, the next question was what happens to the ATP produced by mitochondria isolated in normoxia versus hypoxia? $\mathrm{Ca}^{2+}$ enters the mitochondrial matrix through the protein complex MCU (Patron et al., 2013). $\mathrm{Ca}^{2+}$ is known to activate various dehydrogenases like NADH in the ETC and in turn can enhance the production of ATP (Territo et al., 2001). With this theory in mind, the ATP levels of the mitochondria isolated in normoxia and hypoxia which were also stimulated with succinate/ADP as well as $\mathrm{Ca}^{2+}$ were tested. It was seen that the mitochondria isolated in normoxia which were stimulated with both $\mathrm{Ca}^{2+}$ and succinate/ADP produced higher amounts of ATP compared to non-stimulated mitochondria. However, the mitochondria isolated in hypoxia, which were stimulated with $\mathrm{Ca}^{2+}$ and succinate/ADP, produced higher amounts of ATP than the mitochondria isolated in normoxia. These data together with earlier results indicate that the electron flow from the mitochondria isolated in hypoxia might be very tightly and efficiently regulated which not only results into lower ROS production but also higher ATP production. This is in line with the observed lower complex III activity. The lower oxygen availability in this case favors the tight flow of electrons from the ETC complexes to the direct conversion of oxygen to water rather than compensating more electrons leaking through the complex III in case of higher oxygen availability and oxidized environment. Here, it should also be noted that the enzyme cytochrome $\mathrm{c}$ has a very low $\mathrm{Km}$ value i.e. it has a very high affinity towards molecular oxygen (E Gnaiger, B Lassnig, A Kuznetsov, 1998). Cytochrome $c$ is the enzyme which is responsible to accept the terminal electron and transfer it to the molecular oxygen from ferrocytochrome $\mathrm{c}$, which in turn forms the proton gradient and governs the production of ATP. In line with the described high oxygen affinity and according to the ATP levels observed, in my experimental set up, $0.1 \%$ oxygen concentration is not compromising ATP synthesis. 
The relation between $\mathrm{Ca}^{2+}$ and ATP points towards an altered functionality of $\mathrm{MCU}$ in normoxia and hypoxia. MCU is a protein supercomplex consisting of more than one protein subunit. MCU core protein, MCUb, EMRE, MICU1, MICU2 are part of the protein complex (De Stefani et al., 2014). As described in the results, differences in the ATP production of the mitochondria isolated in normoxia or hypoxia upon stimulating them with $\mathrm{Ca}^{2+}$ were observed. Therefore, MCU supercomplex formation in normoxia and hypoxia was studied using BN-PAGE. Unlike SDS-PAGE, BN-PAGE does not contain any detergent which would break apart protein supercomplexes. It works on a principle wherein the entire protein complex gets a negative charge from the Coomassie blue dye and thus can migrate through the gel towards the positive charge until it reaches its pore size limit according to its molecular weight (Wittig et al., 2006). Therefore, BN-PAGE is useful to study large protein supercomplexes like in this case MCU.

In an earlier study, there was a direct effect if oxidative stress on the MCU complex demonstrated (Dong et al., 2017). In this study, the authors have observed that oxidative stress affects one of the cysteine residues (Cys97) of the human MCU. This reactive thiol group undergoes S-glutathionylation upon oxidation. Due to this oxidation, MCU forms higher order oligomers which promote more $\mathrm{Ca}^{2+}$ uptake from MCU to the mitochondrial matrix. In my study while investigating the complex formation of MCU in mitochondria isolated in normoxia and hypoxia, there were some observable differences in the MCU complex on a BN-PAGE. It was seen that the higher molecular weight MCU components appear to be more in quantity in the mitochondria isolated in hypoxia. This might be due to the fact that there are differences observed in the MCU complex formation strategy in the mitochondria isolated in hypoxia. If this finding relates to the redox changes in the MCU protein described in the literature still needs to be analyzed in more detail.

In conclusion to the second main objective of my thesis, I could demonstrate that physiological oxygenation does have a great impact on the $E_{\mathrm{GSH}}$ of the mitochondrial matrix. Not only the redox homeostasis but also other factors in the mitochondria like the components of ETC and the overall mitochondrial productivity are affected (Fig. 33). Therefore, while performing any kind of studies on isolated mitochondria, it should be taken into consideration that it is highly important to maintain the physiological oxygen environment for proper mitochondrial function. 

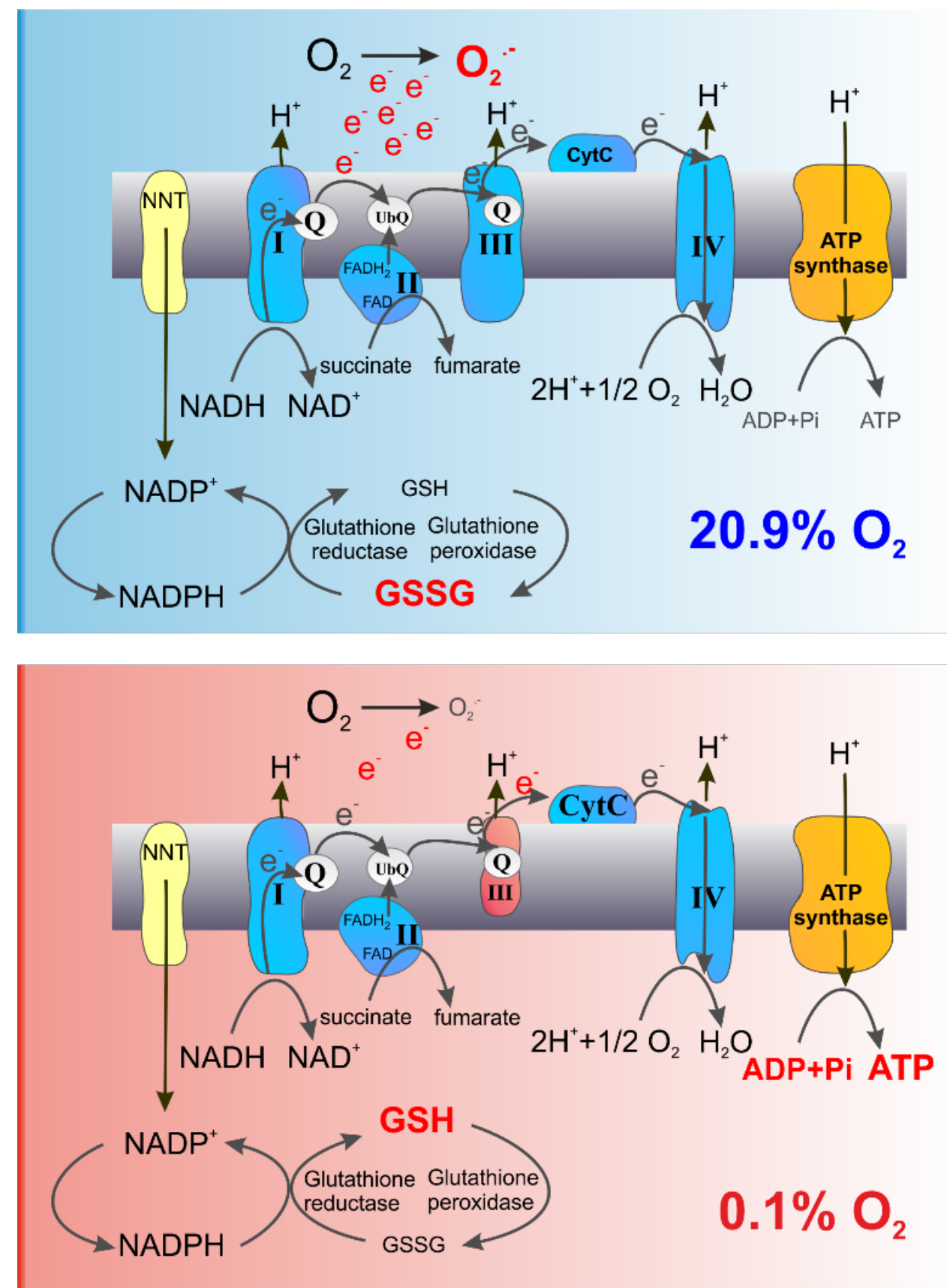

Figure 33: Schematic representation of the observed differences in the components of mitochondria and the mitochondrial ETC when isolated in $20.9 \% \mathrm{O}_{2}$ versus $0.1 \% \mathrm{O}_{2}$.

\subsection{Genetically engineered endogenous $\mathrm{H}_{2} \mathrm{O}_{2}$ producer and biosensor: DAAO-HyPer}

\subsubsection{Local production of $\mathrm{H}_{2} \mathrm{O}_{2}$ in a cell: a necessity}

$\mathrm{H}_{2} \mathrm{O}_{2}$ is one of the ROS primarily produced inside a cell and is in contrast to other species relatively stable. It also has the capacity to pass through biological membranes (Winterbourn, 2008). Like other ROS, $\mathrm{H}_{2} \mathrm{O}_{2}$ is also buffered by the antioxidants in the cell. Therefore, $\mathrm{H}_{2} \mathrm{O}_{2}$ when produced in cells mostly acts locally (Mishina et al., 2011). In most of the studies with 
respect to effects of $\mathrm{H}_{2} \mathrm{O}_{2}$ in a cell, oxidants are provided externally when mimicking increased $\mathrm{H}_{2} \mathrm{O}_{2}$ production. When the $\mathrm{H}_{2} \mathrm{O}_{2}$ is added externally, there is no control over how much of the added concentration actually enters the cell. Not only that, but also the added concentration is most often not in the physiological range and is flooding different subcellular compartments. In summary, the scientific field is lacking a technical tool to stimulate endogenous $\mathrm{H}_{2} \mathrm{O}_{2}$ production in a defined manner. To fill this gap, recently the DAAO-HyPer fusion protein was developed. Use of this ROS producer/biosensor is mostly reported in ex vivo models so far. For example, DAAO was expressed in the HeLa-Kyoto and NIH-3T3 cells (Matlashov et al., 2014). In this study, the authors could very precisely show how much $\mathrm{H}_{2} \mathrm{O}_{2}$ can be produced locally upon stimulation with varied concentrations of the DAAO substrate D-alanine. Further, it was demonstrated that the construct can be localized to a desired organelle and the effects of $\mathrm{H}_{2} \mathrm{O}_{2}$ can be studied in subcellular domains (Bogdanova et al., 2017).

In the $3^{\text {rd }}$ main objective of my thesis, my goal was to generate a transgenic mouse model containing the DAAO-HyPer biosensor and to perform primary characterization of the same. I was successful to generate one mouse line, in which DAAO is located in the nucleus of cardiomyocytes. The attempt to produce a transgenic mouse line, in which DAAO is located in the cytoplasm failed. During screening of the transgenic founder lines, my first task was to confirm the presence of the transgene through genotyping. Once that was confirmed, the cardiomyocytes were isolated and visualized which revealed the presence of a fluorescence signal in the HyPer excitation spectra. Isolated cardiomyocytes were also tested for the presence of DAAO by activating the enzyme through addition of D-alanine. In the case of the NES DAAO-HyPer, there were mice harboring the biosensor confirmed by genotyping. However, the expression was probably so less that a useful positive founder line was not identified. This could be due to dilution of the DAAO-HyPer in the cytosol, which regarding volume is larger than the nucleus. Indeed, I could successfully find one positive founder line in the case of NLS DAAO-HyPer mice. The cardiomyocytes isolated from these mice not only had a strong HyPer expression in the nucleus but also responded dose-dependently to the substrate D-alanine.

Undoubtedly, the DAAO-HyPer biosensor can prove to hold great potential to study the effects of ROS for the development of cardiac dysfunctions. However, some considerations should be taken into account. During the catalysis of the D-alanine, DAAO also produces $\mathrm{NH}_{3}$ in equal amounts as $\mathrm{H}_{2} \mathrm{O}_{2}$. $\mathrm{NH}_{3}$ is present in the human body in higher amounts than the amount produced by the DAAO. Thus, increase in ammonia to an extent of the small concentration produced by DAAO should not have any significant effect. Also any cellular effect that is supposed to be observed by ammonia depends on high ammonia concentrations, i.e. $>0.5 \mathrm{mM}$ 
as compared to the effect given by the amount of $\mathrm{H}_{2} \mathrm{O}_{2}$ being produced by DAAO (Braissant, 2015). It's also known that D-amino acids are present in some mammalian systems in minor amounts (Sakaue et al., 2017). The role of these endogenous amino acids is still not fully clear. However, the amount of D-alanine required for the activation of DAAO is quite high as compared to the endogenous concentration and the experiments can be controlled by adding L-alanine, which is not stimulating the DAAO reaction.

\subsubsection{Role of $\mathrm{H}_{2} \mathrm{O}_{2}$ in development of cardiac dysfunctions}

As described earlier, there was a study performed on the role of $\mathrm{H}_{2} \mathrm{O}_{2}$ for the development of pathophysiological conditions in the rat heart where the authors used a DAAO-Hyper construct (Steinhorn et al., 2018). DAAO-HyPer was expressed in the heart by AAV mediated transduction. The fusion protein was not-targeted to a specific subcellular compartment in the cardiomyocytes. In this study, it was observed that the generation of $\mathrm{H}_{2} \mathrm{O}_{2}$ induces a dilated cardiomyopathy with high systolic dysfunction. Various oxidative stress markers like the targets of the transcription factors $\mathrm{Nrf2}$ and $\mathrm{NF} \kappa \mathrm{B}$ were tested. Upon in vitro activation of DAAO in isolated cardiomyocytes, these genes were found to have a high expression level. When the DAAO was activated in vivo, the animals developed systolic dysfunction with a reduced EF within two weeks of $D$-alanine treatment. Thus, this biosensor proved to be a useful in vivo tool to study the effects of ROS on cardiac dysfunctions.

In case of the mouse model generated during this thesis project, the DAAO is expressed in the nucleus of cardiomyocytes. The very obvious question to target with this mouse model is the source and nature of ROS for heart failure development. In this regard, it is important to note that in the newly generated cardiomyocyte-specific DAAO-HyPer mice the fusion protein is targeted to the nucleus. Histone deacytylases (HDACs) are enzymes which deacytylase histones around the DNA and in turn lead to transcriptional repression (Grozinger and Schreiber, 2002). Upon hypertrophic stimuli the deacetylases are phosphorylated to phosphoHDACs in cardiomyocytes. This phosphorylation takes place by HDAC kinase on the conserved serine residues of HDAC (Vega et al., 2004). The phosphorylation induces the translocation of HDAC from the nucleus to the cytoplasm thus releasing the negative constraints on hypertrophy related transcription factors like MEF2 and NFAT, finally leading to hypertrophy. However, other than phosphorylating the serine residues, HDAC can also translocate from the nucleus by oxidation of specific cysteine residues. For example, it was shown that a ROS generating-hypertrophy stimulus like phenylephrine can oxidize HDAC4 at Cys274/Cys276 and in turn thioredoxin 1 (Trx1) facilitates the reduction of the cysteine residues attenuating cardiac hypertrophy (Ago et al., 2008). In another study, it was 
demonstrated that phenylephrine induced ROS generation in the nucleus might be sourced by NOX4 localized in the nuclear membrane which finally leads to upregulation of hypertrophic stimuli (Matsushima et al., 2013). Thus there is some correlation shown with cardiac hypertrophy and nuclear derived ROS already. However, in this model external stimuli to activate NOX and generate ROS were used. The newly generated aMHC-nucleus DAAOHyPer transgenic mice allow a controlled endogenous $\mathrm{H}_{2} \mathrm{O}_{2}$ production. Therefore, it is definitely tempting in a follow up project to study the effects of $\mathrm{H}_{2} \mathrm{O}_{2}$ produced in the nucleus of cardiomyocytes and the consequences for the development of cardiac hypertrophy. 


\section{Conclusion and outlook}

In the first part of my thesis, I characterized the transgenic cardiomyocyte-specific mouse model Grx1-roGFP2. These biosensor mice were subsequently used to study the effects of aging on the $E_{\mathrm{GSH}}$. Since the biosensor was present in two different compartments in the cell, they were used to analyze the $E_{\mathrm{GSH}}$ in both in two independent mouse lines. During this study, the first striking observation was that the cytoplasm and the mitochondrial matrix of the cardiomyocytes possess a completely independent $E_{\mathrm{GSH}}$ from each other. The mitochondrial matrix is highly reduced whereas the cytoplasm of the cardiomyocytes is more oxidized. However, during aging it was observed that this compartmentalization disappears. The mitochondrial matrix stays reduced but the cytoplasm gets as reduced as the mitochondrial matrix.

In the second main part of my thesis, I utilized the mito Grx1-roGFP2 transgenic mouse model to study the importance of maintaining the physiological oxygenation in order to preserve the redox status of the mitochondrial matrix. Besides the redox status, the functionality of the mitochondria when isolated in an oxygen variable environment was also characterized. The obtained data helped to unravel that the physiological oxygen concentration is highly essential for the mitochondria to maintain their reduced redox status. Not only the redox nature but also the other mitochondrial functionality parameters like the ETC, ROS and ATP levels are all interlinked. In this line it was found that the oxygen concentration plays an important role to maintain all these parameters intact and to play their functional roles as necessary. During the course of this investigation, a possible future interesting study component was detected which is the MCU. The preliminary set of experiments point out that there are differences in the MCU complex formation in normoxia versus hypoxia.

In the third part of my thesis, a novel mouse model specific to cardiomyocytes called the DAAO-HyPer mouse model was generated. In this mouse model, two independent mouse lines: DAAO-HyPer (NES) and DAAO-Hyper (NLS) were generated. In the characterization process, I could find one positive founder line from the DAAO-HyPer NLS mouse line. The checkpoints to confirm this mouse line as a positive founder line included: presence of the transgene confirmed through genotyping, visualization of a HyPer based fluorescent signal in the nucleus by using epifluorescence microscopy, testing the response of the HyPer after activation of DAAO enzyme by the substrate D-alanine. Along with the characterization, the heart function of the transgenic mice were also recorded over time by echocardiography. This helped to verify that the heart function of the transgenic mice was not affected by the transgene itself. The DAAO-Hyper (NLS) mouse model holds a huge potential to study the direct link between ROS generated in the nucleus and cardiac dysfunctions. 


\section{References}

Ago, T., Liu, T., Zhai, P., Chen, W., Li, H., Molkentin, J. D., Vatner, S. F. and Sadoshima, J. (2008). A Redox-Dependent Pathway for Regulating Class II HDACs and Cardiac Hypertrophy. Cell 133, 978-993.

Albrecht, S. C., Barata, A. G., Großhans, J., Teleman, A. A. and Dick, T. P. (2011). In vivo mapping of hydrogen peroxide and oxidized glutathione reveals chemical and regional specificity of redox homeostasis. Cell Metab.

Altenhöfer, S., Kleikers, P. W. M., Radermacher, K. A., Scheurer, P., Hermans, J. J. R., Schiffers, P., Ho, H., Wingler, K. and Schmidt, H. H. H. W. (2012). The NOX toolbox: Validating the role of NADPH oxidases in physiology and disease. Cell. Mol. Life Sci. 69, 2327-2343.

Aoyama, K. and Nakaki, T. (2015). Glutathione in cellular Redox homeostasis: Association with the excitatory amino acid carrier 1 (EAAC1). Molecules.

Back, P., De Vos, W. H., Depuydt, G. G., Matthijssens, F., Vanfleteren, J. R. and Braeckman, B. P. (2012). Exploring real-time in vivo redox biology of developing and aging Caenorhabditis elegans. Free Radic. Biol. Med.

Backs, J., Song, K., Bezprozvannaya, S., Chang, S. and Olson, E. N. (2006). CaM kinase II selectively signals to histone deacetylase 4 during cardiomyocyte hypertrophy. J. Clin. Invest. 116, 1853-1864.

Bedard, K. and Krause, K.-H. (2007). The NOX Family of ROS-Generating NADPH Oxidases: Physiology and Pathophysiology. Physiol. Rev. 87, 245-313.

Belousov, V. V., Fradkov, A. F., Lukyanov, K. A., Staroverov, D. B., Shakhbazov, K. S., Terskikh, A. V. and Lukyanov, S. (2006). Genetically encoded fluorescent indicator for intracellular hydrogen peroxide. Nat. Methods.

Bilan, D. S., Lukyanov, S. A. and Belousov, V. V. (2015). Genetically encoded fluorescent sensors for redox processes. Russ. J. Bioorganic Chem.

Birch-Machin, M. A. and Bowman, A. (2016). Oxidative stress and ageing. Br. J. Dermatol. 175, 26-29.

Blacker, T. S. and Duchen, M. R. (2016). Investigating mitochondrial redox state using NADH and NADPH autofluorescence. Free Radic. Biol. Med.

Bleier, L. and Dröse, S. (2013). Superoxide generation by complex III: From mechanistic 
rationales to functional consequences. Biochim. Biophys. Acta - Bioenerg.

Bogdanova, Y. A., Schultz, C. and Belousov, V. V. (2017). Local Generation and Imaging of Hydrogen Peroxide in Living Cells. Curr. Protoc. Chem. Biol. 9, 117-127.

Braissant, O. (2015). Ammonia toxicity to the brain. Curr. Approach to Hyperammonemia $40-53$.

Breckwoldt, M. O., Pfister, F. M. J., Bradley, P. M., Marinković, P., Williams, P. R., Brill, M. S., Plomer, B., Schmalz, A., St Clair, D. K., Naumann, R., et al. (2014). Multiparametric optical analysis of mitochondrial redox signals during neuronal physiology and pathology in vivo. Nat. Med.

Chen, Q., Vazquez, E. J., Moghaddas, S., Hoppel, C. L. and Lesnefsky, E. J. (2003). Production of reactive oxygen species by mitochondria: Central role of complex III. J. Biol. Chem.

Chen, G., Xie, Y., Deng, J., Liu, Y., Shen, P., Wu, B. and Jiang, C. (2013). Cloning \& Transgenesis D-Amino Acid Oxidase and Metagenomics. 2, 2-4.

De Duve, C. and Baudhuin, P. (2017). Peroxisomes (microbodies and related particles). Physiol. Rev. 46, 323-357.

De Stefani, D., Patron, M. and Rizzuto, R. (2014). Structure and function of the mitochondrial calcium uniporter complex. Biochim. Biophys. Acta - Mol. Cell Res.

Dole, M. (2004). The Natural History of Oxygen. J. Gen. Physiol. 49, 5-27.

Dong, Z., Shanmughapriya, S., Tomar, D., Siddiqui, N., Lynch, S., Nemani, N., Breves, S. L., Zhang, X., Tripathi, A., Palaniappan, P., et al. (2017). Mitochondrial Ca2+Uniporter Is a Mitochondrial Luminal Redox Sensor that Augments MCU Channel Activity. Mol. Cell.

Dooley, C. T., Dore, T. M., Hanson, G. T., Jackson, W. C., Remington, S. J. and Tsien, R. Y. (2004). Imaging dynamic redox changes in mammalian cells with green fluorescent protein indicators. J. Biol. Chem.

Dröge, W. (2015). Free Radicals in the Physiological Control of Cell Function. Physiol. Rev.

E Gnaiger, B Lassnig, A Kuznetsov, G. R. and R. M. (1998). EXCESS CAPACITY OF CYTOCHROME c OXIDASE. J. Exp. Biol. 1139, 1129-1139.

El Benna, J., Faust, L. R. P., Johnson, J. L. and Babior, B. M. (1996). Phosphorylation of the burst oxidase subunit p47phoxas determined by two-dimensional phosphopeptide 
mapping: Phosphorylation by protein kinase $\mathrm{C}$, protein kinase $\mathrm{A}$, and a mitogenactivated protein kinase. J. Biol. Chem.

Espinosa-Diez, C., Miguel, V., Mennerich, D., Kietzmann, T., Sánchez-Pérez, P., Cadenas, S. and Lamas, S. (2015). Antioxidant responses and cellular adjustments to oxidative stress. Redox Biol.

Esterházy, D., King, M. S., Yakovlev, G. and Hirst, J. (2008). Production of reactive oxygen species by complex I (NADH:ubiquinone oxidoreductase) from Escherichia coli and comparison to the enzyme from mitochondria. Biochemistry.

Fearnley, I. M., Walker, J. E., Carroll, J., Shannon, R. J. and Hirst, J. (2003). Analysis of the Subunit Composition of Complex I from Bovine Heart Mitochondria. Mol. Cell. Proteomics.

Fernandes, A. P. and Holmgren, A. (2004). Glutaredoxins: Glutathione-Dependent Redox Enzymes with Functions Far Beyond a Simple Thioredoxin Backup System. Antioxid. Redox Signal.

Forman, H. J., Zhang, H. and Rinna, A. (2009). Glutathione: Overview of its protective roles, measurement, and biosynthesis. Mol. Aspects Med.

Fujikawa, Y., Roma, L. P., Sobotta, M. C., Rose, A. J., Diaz, M. B., Locatelli, G., Breckwoldt, M. O., Misgeld, T., Kerschensteiner, M., Herzig, S., et al. (2016). Mouse redox histology using genetically encoded probes. Sci. Signal. 9, 1-11.

Fukai, T. and Ushio-Fukai, M. (2011). Superoxide Dismutases: Role in Redox Signaling, Vascular Function, and Diseases. Antioxid. Redox Signal. 15, 1583-1606.

Gambardella, J., Sorriento, D., Ciccarelli, M., Del Giudice, C., Fiordelisi, A., Napolitano, L., Trimarco, B., laccarino, G. and Santulli, G. (2017). Mitochondrial Dynamics in Cardiovascular Medicine. Adv. Exp. Med. Biol. 982, 191-202.

Garcia, J., Han, D., Sancheti, H., Yap, L. P., Kaplowitz, N. and Cadenas, E. (2010). Regulation of mitochondrial glutathione redox status and protein glutathionylation by respiratory substrates. J. Biol. Chem.

Girgis, H. S., Bao, X. R., McCombs, J. E., Mootha, V. K., Gohil, V. M., Perocchi, F. and Palmer, A. E. (2010). MICU1 encodes a mitochondrial EF hand protein required for Ca2+ uptake. Nature 467, 291-296.

Glancy, B. and Balaban, R. S. (2012). Role of mitochondrial Ca2+in the regulation of cellular energetics. Biochemistry 51, 2959-2973. 
Griendling, K. K., Touyz, R. M., Zweier, J. L., Dikalov, S., Chilian, W., Chen, Y. R., Harrison, D. G. and Bhatnagar, A. (2016). Measurement of Reactive Oxygen Species, Reactive Nitrogen Species, and Redox-Dependent Signaling in the Cardiovascular System: A Scientific Statement from the American Heart Association. Circ. Res.

Grozinger, C. M. and Schreiber, S. L. (2002). Deacetylase enzymes: Biological functions and the use of small-molecule inhibitors. Chem. Biol. 9, 3-16.

Hafstad, A. D., Nabeebaccus, A. A. and Shah, A. M. (2013). Novel aspects of ROS signalling in heart failure. Basic Res. Cardiol. 108,.

Halliwell, B. (2015). Free Radicals and Other Reactive Species in Disease. In eLS, .

Hanson, G. T., Aggeler, R., Oglesbee, D., Cannon, M., Capaldi, R. A., Tsien, R. Y. and Remington, S. J. (2004). Investigating Mitochondrial Redox Potential with Redoxsensitive Green Fluorescent Protein Indicators. J. Biol. Chem.

Hekimi, S. (2013). Enhanced immunity in slowly aging mutant mice with high mitochondrial oxidative stress. Oncoimmunology 2, 1-2.

Heymes, C., Bendall, J. K., Ratajczak, P., Cave, A. C., Samuel, J. L., Hasenfuss, G. and Shah, A. M. (2003). Increased myocardial NADPH oxidase activity in human heart failure. J. Am. Coll. Cardiol.

Holmgren, A., Johansson, C., Berndt, C., Lonn, M., Hudemann, C. and Lillig, C. (2005). Thiol redox control via thioredoxin and glutaredoxin systems. Biochem. Soc. Trans.

Hyung, S. C., Jun, W. K., Cha, Y. N. and Kim, C. (2006). A quantitative nitroblue tetrazolium assay for determining intracellular superoxide anion production in phagocytic cells. J. Immunoass. Immunochem.

Ighodaro, O. M. and Akinloye, O. A. (2017). First line defence antioxidants-superoxide dismutase (SOD), catalase (CAT) and glutathione peroxidase (GPX): Their fundamental role in the entire antioxidant defence grid. Alexandria J. Med. 54, 287-293.

Kalyanaraman, B., Darley-Usmar, V., Davies, K. J. A., Dennery, P. A., Forman, H. J., Grisham, M. B., Mann, G. E., Moore, K., Roberts, L. J. and Ischiropoulos, H. (2012). Measuring reactive oxygen and nitrogen species with fluorescent probes: Challenges and limitations. Free Radic. Biol. Med.

Keeley, T. P. and Mann, G. E. (2018). Defining Physiological Normoxia for Improved Translation of Cell Physiology to Animal Models and Humans. Physiol. Rev. 99, 161234. 
Kirichok, Y., Krapivinsky, G. and Clapham, D. E. (2004). The mitochondrial calcium uniporter is a highly selective ion channel. Nature 427, 360-364.

Köhler, A. C., Sag, C. M. and Maier, L. S. (2014). Reactive oxygen species and excitationcontraction coupling in the context of cardiac pathology. J. Mol. Cell. Cardiol. 73, 92102.

Korshunov, S. S., Skulachev, V. P. and Starkov, A. A. (1997). High protonic potential actuates a mechanism of production of reactive oxygen species in mitochondria. FEBS Lett. 416, 15-18.

Kudin, P., Bimpong-Buta, B., Vielhaber, S., Elger, E. and Kunz, S. (2004). Characterization of Superoxide-producing Sites in Isolated Brain Mitochondria. J. Biol. Chem.

Kurtcuoglu, V., Hoogewijs, D., Wenger, R., Scholz, C. and Marti, H. (2015). Frequently asked questions in hypoxia research. Hypoxia 35.

Lambeth, J. D. (2004). NOX enzymes and the biology of reactive oxygen. Nat. Rev. Immunol.

Lassègue, B., San Martín, A. and Griendling, K. K. (2012). Biochemistry, physiology, and pathophysiology of NADPH oxidases in the cardiovascular system. Circ. Res.

Liguori, I., Russo, G., Curcio, F., Bulli, G., Aran, L., Della-Morte, D., Gargiulo, G., Testa, G., Cacciatore, F., Bonaduce, D., et al. (2018). Oxidative stress, aging, and diseases. Clin. Interv. Aging.

Looi, Y. H., Grieve, D. J., Siva, A., Walker, S. J., Anilkumar, N., Cave, A. C., Marber, M., Monaghan, M. J. and Shah, A. M. (2008). Involvement of Nox2 NADPH oxidase in adverse cardiac remodeling after myocardial infarction. Hypertension 51, 319-325.

Loschen, G., Flohé, L. and Chance, B. (1971). Respiratory chain linked H2O2 production in pigeon heart mitochondria. FEBS Lett.

Lukyanov, K. A. and Belousov, V. V. (2014). Genetically encoded fluorescent redox sensors. Biochim. Biophys. Acta - Gen. Subj.

Mantoan, M., Checchetto, V., Szabò, I., De Stefani, D., Patron, M., Rizzuto, R., Teardo, E., Granatiero, V., Vecellio Reane, D. and Raffaello, A. (2014). MICU1 and MICU2 Finely Tune the Mitochondrial Ca2+ Uniporter by Exerting Opposite Effects on MCU Activity. Mol. Cell 53, 726-737.

Matlashov, M. E., Belousov, V. V. and Enikolopov, G. (2014). How Much $\mathrm{H}_{2} \mathrm{O}_{2}$ Is 
Produced by Recombinant D-Amino Acid Oxidase in Mammalian Cells? Antioxid. Redox Signal.

Matsushima, S., Kuroda, J., Ago, T., Zhai, P., Park, J. Y., Xie, L. H., Tian, B. and Sadoshima, J. (2013). Increased oxidative stress in the nucleus caused by Nox4 mediates oxidation of HDAC4 and cardiac hypertrophy. Circ. Res.

Meister, A. (1988). Glutathione metabolism and its selective modification. J. Biol. Chem.

Meyer, A. J. and Dick, T. P. (2010). Fluorescent Protein-Based Redox Probes. Antioxid. Redox Signal.

Meyer, A. J., Brach, T., Marty, L., Kreye, S., Rouhier, N., Jacquot, J. P. and Hell, R. (2007). Redox-sensitive GFP in Arabidopsis thaliana is a quantitative biosensor for the redox potential of the cellular glutathione redox buffer. Plant $\mathrm{J}$.

Millis, K. K., Weaver, K. H. and Rabenstein, D. L. (1993). Oxidation/Reduction Potential of Glutathione. J. Org. Chem.

Mishina, N. M., Tyurin-Kuzmin, P. A., Markvicheva, K. N., Vorotnikov, A. V., Tkachuk, V. A., Laketa, V., Schultz, C., Lukyanov, S. and Belousov, V. V. (2011). Does Cellular Hydrogen Peroxide Diffuse or Act Locally? Antioxid. Redox Signal. 14, 1-7.

Morgan, B., Van Laer, K., Owusu, T. N. E., Ezeriṇa, D., Pastor-Flores, D., Amponsah, P. S., Tursch, A. and Dick, T. P. (2016). Real-time monitoring of basal H2O2levels with peroxiredoxin-based probes. Nat. Chem. Biol.

Morrison, D. A. (2009). The Timetree of Life. Syst. Biol. 58, 461-462.

Muller, F. L., Roberts, A. G., Bowman, M. K. and Kramer, D. M. (2003). Architecture of the Q-o site of the cytochrome bc1 complex probed by superoxide production. Biochemistry.

Murphy, M. P. (2008). How mitochondria produce reactive oxygen species. Biochem. J.

Murphy, M. P., Pehar, M., Ross, M. F., Hagen, T. M., Beckman, J. S., Janes, M. S., Robinson, K. M. and Monette, J. S. (2006). Selective fluorescent imaging of superoxide in vivo using ethidium-based probes. Proc. Natl. Acad. Sci.

Nauseef, W. M. (2017). The neutrophil NADPH oxidase. In Hydrogen Peroxide Metabolism in Health and Disease, .

Orr, W. C. and Sohal, R. S. (1994). Extension of life-span by overexpression of superoxide dismutase and catalase in Drosophila melanogaster. Science (80-. ).

Østergaard, H., Henriksen, A., Hansen, F. G. and Winther, J. R. (2001). Shedding light on 
disulfide bond formation: Engineering a redox switch in green fluorescent protein. $E M B O \mathrm{~J}$.

Patel, R., Rinker, L., Peng, J. and Chilian, W. M. (2018). Reactive Oxygen Species: The Good and the Bad. In Reactive Oxygen Species (ROS) in Living Cells, .

Patron, M., Raffaello, A., Granatiero, V., Tosatto, A., Merli, G., Stefani, D. De, Wright, L., Pallafacchina, G., Terrin, A., Mammucari, C., et al. (2013). The mitochondrial calcium uniporter (MCU): Molecular identity and physiological roles. J. Biol. Chem. 288, 1075010758.

Pérez, V. I., Van Remmen, H., Bokov, A., Epstein, C. J., Vijg, J. and Richardson, A. (2009). The overexpression of major antioxidant enzymes does not extend the lifespan of mice. Aging Cell.

Petrungaro, C., Zimmermann, K. M., Küttner, V., Fischer, M., Dengjel, J., Bogeski, I. and Riemer, J. (2015). The Ca2+-dependent release of the Mia40-induced MICU1-MICU2 dimer from MCU regulates mitochondrial Ca2+ uptake. Cell Metab. 22, 721-733.

Phaniendra, A., Jestadi, D. B. and Periyasamy, L. (2015). Free Radicals: Properties, Sources, Targets, and Their Implication in Various Diseases. Indian J. Clin. Biochem. $30,11-26$.

Picard, M., Ritchie, D., Wright, K. J., Romestaing, C., Thomas, M. M., Rowan, S. L., Taivassalo, T. and Hepple, R. T. (2010). Mitochondrial functional impairment with aging is exaggerated in isolated mitochondria compared to permeabilized myofibers. Aging Cell 9, 1032-1046.

Pollegioni, L., Langkau, B., Tischer, W., Ghisla, S. and Pilone, M. S. (1993). Kinetic mechanism of D-amino acid oxidases from Rhodotorula gracilis and Trigonopsis variabilis. J. Biol. Chem. 268, 13850-13857.

Ray, P. D., Huang, B. W. and Tsuji, Y. (2012). Reactive oxygen species (ROS) homeostasis and redox regulation in cellular signaling. Cell. Signal.

Roelofs, B. A., Ge, S. X., Studlack, P. E. and Polster, B. M. (2015). Low micromolar concentrations of the superoxide probe MitoSOX uncouple neural mitochondria and inhibit complex IV. Free Radic. Biol. Med.

Rottenberg, H., Covian, R. and Trumpower, B. L. (2009). Membrane potential greatly enhances superoxide generation by the cytochrome bc1 complex reconstituted into phospholipid vesicles. J. Biol. Chem. 
Rydström, J. (2006). Mitochondrial NADPH, transhydrogenase and disease. Biochim. Biophys. Acta - Bioenerg. 1757, 721-726.

Sakaue, H., Hamase, K., Koga, R., Miyoshi, Y. and Konno, R. (2017). Mouse d-AminoAcid Oxidase: Distribution and Physiological Substrates. Front. Mol. Biosci. 4, 1-10.

Schriner, S. E., Linford, N. J., Martin, G. M., Treuting, P., Ogburn, C. E., Emond, M., Coskun, P. E., Ladiges, W., Wolf, N., Remmen, H. Van, et al. (2009). Extension of Murine Life Span by Overexpression of Catalase Targeted to Mitochondria. 1909, 1909_ 1911.

Semenza, G. L. (2007). Life with oxygen. Science (80-. ).

Sinaasappel, M., Mik, E. G., Beek, J. F., Ince, C., Aten, J. A., Stap, J. and van Leeuwen, T. G. (2006). Mitochondrial PO2 measured by delayed fluorescence of endogenous protoporphyrin IX.

Snoep, J. L., Olivier, B. G., Hofmeyr, J.-H. S., Rohwer, J. M. and Pillay, C. S. (2008). Enzymes or redox couples? The kinetics of thioredoxin and glutaredoxin reactions in a systems biology context. Biochem. J.

Starkov, A. A., Polster, B. M. and Fiskum, G. (2002). Regulation of hydrogen peroxide production by brain mitochondria by calcium and Bax. J. Neurochem.

Steinhorn, B., Sorrentino, A., Badole, S., Bogdanova, Y., Belousov, V. and Michel, T. (2018). Chemogenetic generation of hydrogen peroxide in the heart induces severe cardiac dysfunction. Nat. Commun.

Summers, F. A., Zhao, B., Ganini, D. and Mason, R. P. (2013). Photooxidation of amplex red to resorufin: Implications of exposing the amplex red assay to light. In Methods in Enzymology, .

Swain, L., Kesemeyer, A., Meyer-Roxlau, S., Vettel, C., Zieseniss, A., Güntsch, A., Jatho, A., Becker, A., Nanadikar, M. S., Morgan, B., et al. (2016a). Redox Imaging Using Cardiac Myocyte-Specific Transgenic Biosensor Mice. Circ. Res.

Swain, L., Kesemeyer, A., Meyer-Roxlau, S., Vettel, C., Zieseniss, A., Güntsch, A., Jatho, A., Becker, A., Nanadikar, M. S., Morgan, B., et al. (2016b). Redox Imaging Using Cardiac Myocyte-Specific Transgenic Biosensor Mice. Circ. Res. 119,.

Swain, L., Nanadikar, M. S., Borowik, S., Katschinski, D. M. and Zieseniss, A. (2018a). Transgenic Organisms Meet Redox Bioimaging: One Step Closer to Physiology. Antioxid. Redox Signal. 
Swain, L., Nanadikar, M. S., Borowik, S., Zieseniss, A. and Katschinski, D. M. (2018b). Transgenic organisms meet redox bioimaging: One step closer to physiology. Antioxidants Redox Signal. 29,.

Territo, P. R., French, S. A., Dunleavy, M. C., Evans, F. J. and Balaban, R. S. (2001). Calcium activation of heart mitochondrial oxidative phosphorylation. Rapid kinetics of mV்O2, NADH, and light scattering. J. Biol. Chem. 276, 2586-2599.

Tetz, L. M., Kamau, P. W., Cheng, A. A., Meeker, J. D. and Loch-Caruso, R. (2013). Troubleshooting the dichlorofluorescein assay to avoid artifacts in measurement of toxicant-stimulated cellular production of reactive oxidant species. J. Pharmacol. Toxicol. Methods.

Vandewalle, P. L. and Petersen, N. O. (1987). Oxidation of reduced cytochrome c by hydrogen peroxide. Implications for Superoxide assays. FEBS Lett.

Vaupel, P., Kallinowski, F. and Okunieff, P. (1989). Blood Flow, Oxygen and Nutrient Supply, and Metabolic Microenvironment of Human Tumors: A Review. Cancer Res.

Vega, R. B., Harrison, B. C., Meadows, E., Charles, R., Papst, P. J., Olson, E. N., Timothy, A., Roberts, C. R. and Mckinsey, T. A. (2004). Protein Kinases C and D Mediate Agonist-Dependent Cardiac Hypertrophy through Nuclear Export of Histone Deacetylase 5. Mol. Cell. Biol. 24, 8374-8385.

Wachter, R. M., Yarbrough, D., Kallio, K. and Remington, S. J. (2000). Crystallographic and energetic analysis of binding of selected anions to the yellow variants of green fluorescent protein. J. Mol. Biol. 301, 157-171.

Wang, M., Brewer, A. C., Anilkumar, N., Schroder, K., Shah, A. M., Yu, B., Zhang, M., Brandes, R. P., Walker, S. J., Santos, C. X. C., et al. (2010). NADPH oxidase-4 mediates protection against chronic load-induced stress in mouse hearts by enhancing angiogenesis. Proc. Natl. Acad. Sci. 107, 18121-18126.

Wenger, R. H. (2006). Mitochondria: Oxygen sinks rather than sensors? Med. Hypotheses 66, 380-383.

White, C. C., Viernes, H., Krejsa, C. M., Botta, D. and Kavanagh, T. J. (2003). Fluorescence-based microtiter plate assay for glutamate-cysteine ligase activity. Anal. Biochem.

Winterbourn, C. C. (2008). Reconciling the chemistry and biology of reactive oxygen species. Nat. Chem. Biol. 4, 278-286. 
Winther, J. R., Labate, V., Ostergaard, H., Maulucci, G., Pani, G., De Spirito, M., Mele, M., Galeotti, T., Arcovito, G. and Panieri, E. (2008). High-Resolution Imaging of Redox Signaling in Live Cells Through an Oxidation-Sensitive Yellow Fluorescent Protein. Sci. Signal. 1, pl3-pl3.

Wittig, I., Braun, H. P. and Schägger, H. (2006). Blue native PAGE. Nat. Protoc.

Yap, L. P., Garcia, J. V., Han, D. and Cadenas, E. (2009). The energy-redox axis in aging and age-related neurodegeneration. Adv. Drug Deliv. Rev.

Zhou, M., Diwu, Z., Panchuk-Voloshina, N. and Haugland, R. P. (1997). A stable nonfluorescent derivative of resorufin for the fluorometric determination of trace hydrogen peroxide: Applications in detecting the activity of phagocyte NADPH oxidase and other oxidases. Anal. Biochem.

Zielonka, J. and Kalyanaraman, B. (2010). Hydroethidine- and MitoSOX-derived red fluorescence is not a reliable indicator of intracellular superoxide formation: Another inconvenient truth. Free Radic. Biol. Med. 


\section{Acknowledgements}

Firstly, I would like to express my sincere gratitude to my PhD supervisor Prof. Dr. Dörthe M. Katschinski for supporting and guiding me throughout my PhD research duration. I would like to thank her for giving me an opportunity to work in this very exciting project and to develop myself scientifically over the period of time. Her advices and supervision helped me in all of my research time as well as during my thesis writing. I could not have imagined to have a better mentor and advisor than Prof. Katschinski in my PhD life.

Besides my supervisor, I would like to thank my thesis committee members Prof. Dr. Blanche Schwappach and Prof. Dr. Stephan E. Lehnart for their valuable suggestions throughout the project. I would also like to thank Dr. Antje Ebert, Prof. Dr. Heidi Hahn and Prof. Hubertus Jarry for accepting the invitation to be a part of my examination board for my $\mathrm{PhD}$ defense.

I am grateful to have Prof. Dr. Peter Rehling, Prof. Dr. Ivan Bogeski and Prof. Dr. Vsevolod V. Belousov on board as great scientific advisors. My sincere thanks goes to Dr. Lija Swain who trained me to her best during the initial days of my lab work with the special techniques and skills required for the project. I especially would like to express my sincere thanks to Dr. Aline Jatho for providing her expertise to perform echocardiography in my project as well as helped me to learn proper animal handling and animal work related formalities.

My special thanks to Annette Hillemann for providing her excellent technical support throughout the project. I am grateful to have her not only as a support system in lab work but also to have her as a great friend with whom I could built a long lasting connection. I would also like to thank Sören for his technical help during my project. I am glad to receive help during the project from Dr. Jan Dudek, Magnus Hartmann, Ana Vergel and Anzhelika Svetlova.

I would like to express my appreciation to Dr. Gijsbert Jacob van Belle for his very helpful suggestions in my scientific writing skills and for other technical issues, Dr. Anke Zieseniss for her help, and suggestions in my project, Lisa Leinhos to be such a nice colleague, friend, common office room sharer, lunch partner and much more. I would also like to thank Sabine Krull, Katja Brechtel-Curth, Doris Heidenreich for their technical help whenever required. The people whom I would also really like to thank are the coordinators of the IRTG1816 PhD program; Dr. Christina Würtz, Fulya Ören and Ulrike Fischer for all the possible technical support they could offer from their side.

Last but not the least, I would like to thank my family and friends who supported me unconditionally. A very special thanks to my Parents who believed in me and supported me all the way from India during my PhD journey. An exceptional thanks to my dear besties Udhaya Bhaskar and Shilpa Patil for their immense care and emotional support throughout the time. 